\title{
Path combinatorics and light leaves for quiver Hecke algebras
}

\author{
Chris Bowman ${ }^{1} \cdot$ Anton Cox $^{2} \cdot$ Amit Hazi $^{2} \cdot$ Dimitris Michailidis $^{3}$
}

Received: 3 September 2020 / Accepted: 26 June 2021 / Published online: 29 September 2021

(c) The Author(s) 2021

\begin{abstract}
We recast the classical notion of "standard tableaux" in an alcove-geometric setting and extend these classical ideas to all "reduced paths" in our geometry. This broader path-perspective is essential for implementing the higher categorical ideas of Elias-Williamson in the setting of quiver Hecke algebras. Our first main result is the construction of light leaves bases of quiver Hecke algebras. These bases are richer and encode more structural information than their classical counterparts, even in the case of the symmetric groups. Our second main result provides path-theoretic generators for the "Bott-Samelson truncation" of the quiver Hecke algebra.
\end{abstract}

\section{Introduction}

The symmetric group lies at the intersection of two great categorical theories: KhovanovLauda and Rouquier's categorification of quantum groups and their knot invariants $[8,14]$ and Elias-Williamson's diagrammatic categorification in terms of endomorphisms of BottSamelson bimodules. The purpose of this paper and its companion [2] is to construct an explicit isomorphism between these two diagrammatic worlds. The backbone of this isomorphism is provided by the "light-leaves" bases of these algebras.

The light leaves bases of diagrammatic Bott-Samelson endomorphism algebras were crucial in the calculation of counterexamples to the expected bounds of Lusztig's and James' conjectures [15]. These bases are structurally far richer than any known basis of the quiver

Chris Bowman

Chris.Bowman-Scargill@york.ac.uk

Anton Cox

A.G.Cox@ city.ac.uk

Amit Hazi

Amit.Hazi@city.ac.uk

Dimitris Michailidis

D.Michailidis@kent.ac.uk

1 Department of Mathematics, University of York, Heslington, York YO10 5DD, UK

2 Department of Mathematics, City, University of London, London, UK

3 School of Mathematics, Statistics and Actuarial Science, University of Kent, Canterbury, UK 
Hecke algebra - they vary with respect to each possible choice of reduced word/path-vector in the alcove geometry-and this richer structure is necessary in order to construct a basis in terms of the "Soergel 2-generators" of these algebras. In this paper we show that the (quasihereditary quotients of) quiver Hecke algebras, $\mathcal{H}_{n}^{\sigma}$ for $\sigma \in \mathbb{Z}^{\ell}$, have analogues of the light leaves bases in the "non-singular" or "regular" case.

Without loss of generality, we assume that $\sigma \in \mathbb{Z}^{\ell}$ is weakly increasing. We then let $\underline{h}=\left(h_{0}, \ldots, h_{\ell-1}\right) \in \mathbb{N}^{\ell}$ be such that $h_{m} \leqslant \sigma_{m+1}-\sigma_{m}$ for $0 \leqslant m<\ell-1$ and $h_{\ell-1}<$ $\bar{e}+\sigma_{0}-\sigma_{\ell-1}$. The light leaves bases we construct are indexed by paths in an alcove geometry of type

$$
A_{h_{0}-1} \times \cdots \times A_{h_{\ell-1}-1} \backslash \widehat{A}_{h_{0}+\cdots+h_{\ell-1}-1}
$$

for $e>h=h_{0}+\cdots+h_{\ell-1}$, where each point in this geometry corresponds to an $\ell$ multipartition with at most $h_{m}$ columns in the $m$ th component (we denote the set of such $\ell$-multipartitions by $\left.\mathscr{P}_{h}(n)\right)$. In this manner, we obtain cellular bases of the largest possible quasi-hereditary quotients of $\mathcal{H}_{n}^{\sigma}$ controlled by non-singular Kazhdan-Lusztig theory (this is the broadest possible generalisation, in the context of cyclotomic quiver Hecke algebras, of studying the category of tilting modules of the principal block of the general linear group, $\mathrm{GL}_{h}(\mathbb{k})$, in characteristic $\left.p>h\right)$.

Theorem A (Regular light leaves bases for quiver Hecke algebras) For each $\lambda \in \mathscr{P}_{\underline{h}}(n)$ we fix a reduced path $Q_{\lambda} \in \operatorname{Path}_{\underline{h}}(\lambda)$ and for each $\mathrm{S} \in \operatorname{Path}_{\underline{h}}(\lambda)$, we fix an associated reduced path vector $\underline{\mathrm{P}}_{\mathrm{S}}$ terminating with $Q_{\lambda}$ (this notation is defined in Sects. 3 and 2). We have that

$$
\left\{\Upsilon_{\underline{\mathrm{P}}_{\mathrm{S}}^{\mathrm{S}}}^{\mathrm{S}} \Upsilon_{\mathrm{T}}^{\underline{\mathrm{P}}_{\mathrm{T}}} \mid \mathrm{S}, \mathrm{T} \in \operatorname{Path}_{\underline{h}}(\lambda), \lambda \in \mathscr{P}_{\underline{h}}(n)\right\}
$$

is a cellular basis of $\mathcal{H}_{n}^{\sigma} / \mathcal{H}_{n}^{\sigma} \mathrm{y}_{h} \mathcal{H}_{n}^{\sigma}$ where we quotient by the ideal generated by the element $y_{h}$ which kills all simples indexed by $\ell$-partitions with more than $h_{m}$ columns in the mth component.

We then consider the so-called "Bott-Samelson truncations" to the principal block

$$
\mathrm{f}_{n, \sigma}\left(\mathcal{H}_{n}^{\sigma} / \mathcal{H}_{n}^{\sigma} \mathrm{y}_{\underline{h}} \mathcal{H}_{n}^{\sigma}\right) \mathrm{f}_{n, \sigma} \quad \text { for } \quad \mathrm{f}_{n, \sigma}=\sum_{\substack{\mathrm{S} \in \operatorname{Std}_{n, \sigma}(\lambda) \\ \lambda \in \mathscr{P}_{\underline{h}}(n)}} e_{\mathrm{S}}
$$

which we will show (in the companion paper [2]) are isomorphic to the (breadth enhanced) diagrammatic Bott-Samelson endomorphism algebras of Elias-Williamson [6]. The charm of this isomorphism is that it allows one to view the current state-of-the-art regarding $p$ Kazhdan-Lusztig theory (in type $A$ ) entirely within the context of the group algebra of the symmetric group, without the need for calculating intersection cohomology groups, or working with parity sheaves, or appealing to the deepest results of 2-categorical Lie theory. Furthermore, this isomorphism will allow us to prove (in a future paper) the beautiful "categorical blob" conjecture of Libedinsky-Plaza which brings together p-Kazhdan-Lusztig theory and statistical mechanics [11]. In this paper, we specialise Theorem A by making certain path-theoretic choices which allow us to reconstruct Elias-Williamson's generators entirely within the quiver Hecke algebra itself, using our language of paths.

Theorem B The Bott-Samelson truncation of the Hecke algebra $\mathrm{f}_{n, \sigma}\left(\mathcal{H}_{n}^{\sigma} / \mathcal{H}_{n}^{\sigma} \mathrm{y}_{\underline{h}} \mathcal{H}_{n}^{\sigma}\right) \mathrm{f}_{n, \sigma}$ is generated by horizontal and vertical concatenation of the elements

$$
e_{\mathrm{P}_{\alpha}}, \quad \text { fork }_{\alpha \alpha}^{\alpha \emptyset}, \quad \operatorname{spot}_{\alpha}^{\emptyset}, \quad \operatorname{hex}_{\alpha \beta \alpha}^{\beta \alpha \beta}, \quad \operatorname{com}_{\beta \gamma}^{\gamma \beta}, \quad e_{\mathrm{P}_{\emptyset}}, \text { and } \operatorname{adj}_{\alpha \emptyset}^{\emptyset \alpha}
$$


(this notation is defined in Sect. 4) for $\alpha, \beta, \gamma \in \Pi$ such that $\alpha$ and $\beta$ label an arbitrary pair of non-commuting reflections and $\beta$ and $\gamma$ label an arbitrary pair of commuting reflections.

The paper is structured as follows. In Sect. 2 we construct a "classical-type" cellular basis of $\mathcal{H}_{n}^{\sigma} / \mathcal{H}_{n}^{\sigma} \mathrm{y}_{h} \mathcal{H}_{n}^{\sigma}$ in terms of tableaux but using a slightly exotic dominance ordering-the proofs in this section are a little dry and can be skipped on the first reading. In Sect. 3, we upgrade this basis to a "light leaf type" construction and prove Theorem A. Finally, in Sect. 4 of the paper we illustrate how Theorem A allows us to reconstruct the precise analogue of the light leaves basis for the Bott-Samelson endomorphism algebras for regular blocks of quiver Hecke algebras (as a special case of Theorem A, written in terms of the generators of Theorem B). We do this in the exact language used by Elias, Libedinsky, and Williamson in order to make the construction clear for a reader whose background lies in either field.

This paper is a companion to [2], but the reader should note that the results here are entirely self-contained (although we refer to [2] for further development of ideas, examples, etc).

\section{A tableaux basis of the quiver Hecke algebra}

We let $\mathfrak{S}_{n}$ denote the symmetric group on $n$ letters and let $\ell: \mathfrak{S}_{n} \rightarrow \mathbb{Z}$ denotes its length function. We let $\leqslant$ denote the (strong) Bruhat order on $\mathfrak{S}_{n}$. Given $\underline{i}=\left(i_{1}, \ldots, i_{n}\right) \in(\mathbb{Z} / e \mathbb{Z})^{n}$ and $s_{r}=(r, r+1) \in \mathfrak{S}_{n}$ we set $s_{r}(\underline{i})=\left(i_{1}, \ldots, i_{r-1}, i_{r+1}, i_{r}, i_{r+2}, \ldots, i_{n}\right)$.

Definition 2.1 $[3,8,14]$ Fix $e>2$. The quiver Hecke algebra (or KLR algebra), $\mathcal{H}_{n}$, is defined to be the unital, associative $\mathbb{Z}$-algebra with generators

$$
\left\{e_{\underline{i}} \mid \underline{i}=\left(i_{1}, \ldots, i_{n}\right) \in(\mathbb{Z} / e \mathbb{Z})^{n}\right\} \cup\left\{y_{1}, \ldots, y_{n}\right\} \cup\left\{\psi_{1}, \ldots, \psi_{n-1}\right\},
$$

subject to the relations

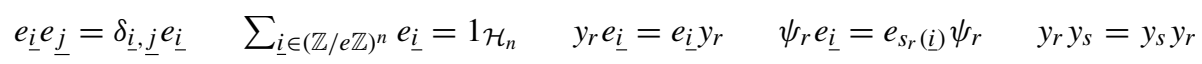

for all $r, s, \underline{i}, j$ and

$$
\begin{aligned}
& \psi_{r} y_{s}=y_{s} \psi_{r} \text { for } s \neq r, r+1 \quad \psi_{r} \psi_{s}=\psi_{s} \psi_{r} \text { for }|r-s|>1 \\
& y_{r} \psi_{r} e_{\underline{i}}=\left(\psi_{r} y_{r+1}-\delta_{i_{r}, i_{r+1}}\right) e_{\underline{i}} \quad y_{r+1} \psi_{r} e_{\underline{i}}=\left(\psi_{r} y_{r}+\delta_{i_{r}, i_{r+1}}\right) e_{\underline{i}} \\
& \psi_{r} \psi_{r} e_{\underline{i}}= \begin{cases}0 & \text { if } i_{r}=i_{r+1}, \\
e_{\underline{i}} & \text { if } i_{r+1} \neq i_{r}, i_{r} \pm 1 \\
\left(y_{r+1}-y_{r}\right) e_{\underline{i}} & \text { if } i_{r+1}=i_{r}+1, \\
\left(y_{r}-y_{r+1}\right) e_{\underline{i}} & \text { if } i_{r+1}=i_{r}-1\end{cases} \\
& \psi_{r} \psi_{r+1} \psi_{r} e_{\underline{i}}= \begin{cases}\left(\psi_{r+1} \psi_{r} \psi_{r+1}-1\right) e_{\underline{i}} & \text { if } i_{r}=i_{r+2}=i_{r+1}+1 \\
\left(\psi_{r+1} \psi_{r} \psi_{r+1}+1\right) e_{\underline{i}} & \text { if } i_{r}=i_{r+2}=i_{r+1}-1 \\
\psi_{r+1} \psi_{r} \psi_{r+1} e_{\underline{i}} & \text { otherwise }\end{cases}
\end{aligned}
$$

for all permitted $r, s, i, j$. We identify such elements with decorated permutations and the multiplication with vertical concatenation, o, of these diagrams in the standard fashion of 


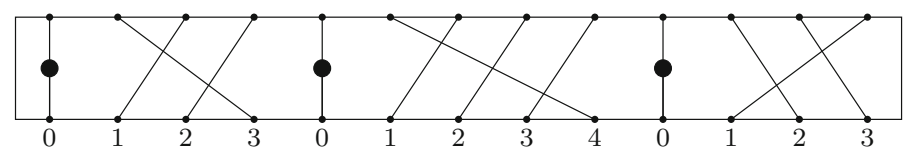

Fig. 1 The element $y_{1} \psi_{4}^{2} e_{(0,1,2,3)} \otimes y_{1} \psi_{5}^{2} e_{(0,1,2,3,4)} \otimes y_{1} \psi_{2}^{4} e_{(0,1,2,3)}$

[3, Section 1] and as illustrated in Fig. 1. We let $*$ denote the anti-involution which fixes the generators.

Definition 2.2 Fix $e>2$ and $\sigma \in \mathbb{Z}^{\ell}$. The cyclotomic quiver Hecke algebra, $\mathcal{H}_{n}^{\sigma}$, is defined to be the quotient of $\mathcal{H}_{n}$ by the relation

$$
y_{1}^{\sharp\left\{\sigma_{m} \mid \sigma_{m}=i_{1}, 1 \leqslant m \leqslant \ell\right\}} e_{\underline{i}}=0 \quad \text { for } \underline{i} \in(\mathbb{Z} / e \mathbb{Z})^{n} .
$$

As we see in Fig. 1, the $y_{k}$ elements are visualised as dots on strands; we hence refer to them as KLR dots. Given $p<q$ we set

$$
\begin{aligned}
w_{q}^{p} & =s_{p} s_{p+1} \cdots s_{q-1} \quad w_{p}^{q}=s_{q-1} \cdots s_{p+1} s_{p} \quad \psi_{q}^{p}=\psi_{p} \psi_{p+1} \cdots \psi_{q-1} \\
\psi_{p}^{q} & =\psi_{q-1} \cdots \psi_{p+1} \psi_{p} .
\end{aligned}
$$

and given an expression $\underline{w}=s_{i_{1}} \cdots s_{i_{p}} \in \mathfrak{S}_{n}$ we set $\psi_{\underline{w}}=\psi_{i_{1}} \cdots \psi_{i_{p}} \in \mathcal{H}_{n}$. We let $\otimes$ denote the horizontal concatenation of KLR diagrams. Finally, we define the degree as follows,

$$
\operatorname{deg}\left(e_{\underline{i}}\right)=0 \quad \operatorname{deg}\left(y_{r}\right)=2 \operatorname{deg}\left(\psi_{r} e_{\underline{i}}\right)= \begin{cases}-2 & \text { if } i_{r}=i_{r+1} \\ 1 & \text { if } i_{r}=i_{r+1} \pm 1 . \\ 0 & \text { otherwise }\end{cases}
$$

\subsection{Box configurations, partitions, residues and tableaux}

For a fixed $n \in \mathbb{N}$ and $\ell \geqslant 1$ we define a box-configuration to be a subset of

$$
\{[i, j, m] \mid 0 \leqslant m<\ell, 1 \leqslant i, j \leqslant n\}
$$

of $n$ elements, which we call boxes, and we let $\mathcal{B}_{\ell}(n)$ denote the set of all such boxconfigurations. We refer to a box $[i, j, m] \in \lambda \in \mathcal{B}_{\ell}(n)$ as being in the $i$ th row and $j$ th column of the $m$ th component of $\lambda$. Given a box, $[i, j, m]$, we define the content of this box to be $\mathrm{ct}[i, j, m]=\sigma_{m}+j-i$ and we define its residue to be $\operatorname{res}[i, j, m]=\mathrm{ct}[i, j, m]$ $(\bmod e)$. We refer to a box of residue $r \in \mathbb{Z} / e \mathbb{Z}$ as an $r$-box.

We define a composition, $\lambda$, of $n$ to be a finite sequence of non-negative integers $\left(\lambda_{1}, \lambda_{2}, \ldots\right)$ whose sum, $|\lambda|=\lambda_{1}+\lambda_{2}+\cdots$, equals $n$. We say that $\lambda$ is a partition if, in addition, this sequence is weakly decreasing. An $\ell$-multicomposition (respectively $\ell$-multipartition) $\lambda=\left(\lambda^{(0)}, \ldots, \lambda^{(\ell-1)}\right)$ of $n$ is an $\ell$-tuple of compositions (respectively partitions) such that $\left|\lambda^{(0)}\right|+\cdots+\left|\lambda^{(\ell-1)}\right|=n$. We denote the sets of $\ell$ multipartitions and $\ell$-multi-compositions of $n$ by $\mathscr{P}_{\ell}(n)$ and $\mathcal{C}_{\ell}(n)$, respectively. Given $\lambda=\left(\lambda^{(0)}, \lambda^{(1)}, \ldots, \lambda^{(\ell-1)}\right) \in \mathscr{P}_{\ell}(n)$, the Young diagram of $\lambda$ is defined to be the box configuration,

$$
\left\{[i, j, m] \mid 1 \leqslant j \leqslant \lambda_{i}^{(m)}, 0 \leqslant m<\ell\right\} .
$$


We do not distinguish between the multipartition and its Young diagram. We let $\varnothing$ denote the empty multipartition. Given $\lambda \in \mathcal{B}_{\ell}(n)$, we let

$$
\begin{aligned}
\operatorname{Rem}(\lambda) & =\{[r, c, m] \in \lambda \mid[r+1, c, m] \notin \lambda,[r, c+1, m] \notin \lambda\} \\
\operatorname{Add}(\lambda) & =\{[r, c, m] \notin \lambda \mid[r-1, c, m] \in \lambda \text { if } r>1,[r, c-1, m] \in \lambda \text { if } c>1\}
\end{aligned}
$$

for $\lambda \in \mathscr{P}_{\ell}(n) \subseteq \mathcal{B}_{\ell}(n)$ this coincides with the usual definition of addable and removable boxes. We let $\operatorname{Rem}_{r}(\lambda) \subseteq \operatorname{Rem}(\lambda)$ and $\operatorname{Add}_{r}(\lambda) \subseteq \operatorname{Add}(\lambda)$ denote the subsets of boxes of residue $r \in \mathbb{Z} / e \mathbb{Z}$.

Given $\lambda \in \mathcal{B}_{\ell}(n)$, we define a $\lambda$ - tableau to be a filling of the boxes of [ $\left.\lambda\right]$ with the numbers $\{1, \ldots, n\}$. For $\lambda \in \mathcal{B}_{\ell}(n)$, we say that a $\lambda$-tableau is row-standard, column-standard, or simply standard if the entries in each component increase along the rows, increase along the columns, or increase along both rows and columns, respectively. We say that a $\lambda$-tableau $\mathrm{S}$ has shape $\lambda$ and write Shape $(\mathrm{S})=\lambda$. Given $\lambda \in \mathcal{B}_{\ell}(n)$, we let $\operatorname{Tab}(\lambda)$ denote the set of all tableaux of shape $\lambda \in \mathcal{B}_{\ell}(n)$. Given $\mathrm{T} \in \operatorname{Tab}(\lambda)$ and $1 \leqslant k \leqslant n$, we let $\mathrm{T}^{-1}(k)$ denote the box $\square \in \lambda$ such that $\mathrm{T}(\square)=k$. We let $\operatorname{RStd}(\lambda), \operatorname{CStd}(\lambda), \operatorname{Std}(\lambda) \subseteq \operatorname{Tab}(\lambda)$ denote the subsets of all row-standard, column-standard, and standard tableaux, respectively. We let $\operatorname{Std}(n)=\cup_{\lambda \in \mathscr{P}_{\ell}(n)} \operatorname{Std}(\lambda)$ for $n \in \mathbb{N}$.

Definition 2.3 We define the reverse cylindric ordering, $\succ$, as follows. Let $1 \leqslant i, i^{\prime}, j, j^{\prime} \leqslant$ $n$ and $0 \leqslant m, m^{\prime}<\ell$. We write $[i, j, m] \succ\left[i^{\prime}, j^{\prime}, m^{\prime}\right]$ if $i<i^{\prime}$, or $i=i^{\prime}$ and $m<m^{\prime}$, or $i=i^{\prime}$ and $m=m^{\prime}$ and $j<j^{\prime}$. For $\lambda, \mu \in \mathcal{B}_{\ell}(n)$, we write $\lambda \succ \mu$ if the $\succ$-minimal box $\square \in(\lambda \cup \mu) \backslash(\lambda \cap \mu)$ belongs to $\mu$.

Definition 2.4 We define the dominance ordering, $\triangleright$, as follows. Let $1 \leqslant i, i^{\prime}, j, j^{\prime} \leqslant n$ and $0 \leqslant m, m^{\prime}<\ell$. We write $[i, j, m] \triangleright\left[i^{\prime}, j^{\prime}, m^{\prime}\right]$ if $m<m^{\prime}$, or $m=m^{\prime}$ and $i<i^{\prime}$, or $i=i^{\prime}$ and $m=m^{\prime}$ and $j<j^{\prime}$. Given $\lambda, \mu \in \mathcal{B}_{\ell}(n)$, we write $\lambda \triangleright \mu$ if the $\triangleright$-maximal box $\square \in(\lambda \cup \mu) \backslash(\lambda \cap \mu)$ belongs to $\lambda$.

Given $\mathrm{S}$, we write $\mathrm{S} \downarrow \leqslant k$ or $\mathrm{S} \downarrow_{\{1, \ldots, k\}}$ (respectively $\mathrm{S} \downarrow \geqslant k$ ) for the subtableau of $\mathrm{S}$ consisting solely of the entries 1 through $k$ (respectively of the entries $k$ through $n$ ). Given $\lambda \in \mathcal{B}_{\ell}(n)$, we let $T_{\lambda}$ denote the $\lambda$-tableau in which we place the entry $n$ in the minimal $\succ$-node of $\lambda$, then continue in this fashion inductively. Given $\lambda \in \mathcal{B}_{\ell}(n)$, we let $S_{\lambda}$ denote the $\lambda$-tableau in which we place the entry $n$ in the minimal $\triangleright$-node of $\lambda$, then continue in this fashion inductively. Finally, given $\mathrm{S}, \mathrm{T}$ two $\lambda$-tableaux, we let $w_{\top}^{\mathrm{S}} \in \mathfrak{S}_{n}$ be the permutation such that $w_{\mathrm{T}}^{\mathrm{S}}(\mathrm{T})=\mathrm{S}$.

Example 2.5 For $\lambda=\left(\left(2,1^{2}\right),\left(2^{2}, 1\right),\left(1^{3}\right)\right)$, we have that $w_{\mathrm{T}_{\lambda}}^{\mathrm{S}}=(4,5)(2,6)$ for $\mathrm{S}$ and $\mathrm{T}_{\lambda}$ the tableaux

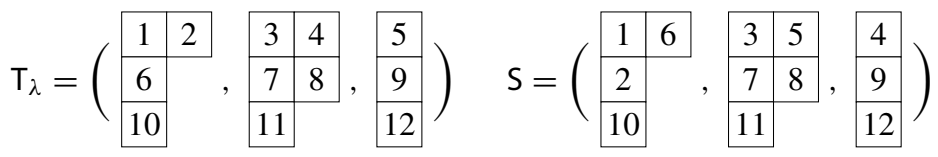

Definition 2.6 Given any box, $[r, c, m]$, we define the associated $(\succ)$-Garnir belt to be the collection of boxes, $\operatorname{Gar}_{\succ}([r, c, m])$, as follows

$\{[r, j, k] \mid j \geqslant 1,1 \leqslant k<m\} \cup\{[r, j, m] \mid 1 \leqslant j \leqslant c\} \cup\{[r-1, j, m] \mid c \leqslant j\} \cup\{[r-1, j, k] \mid j \geqslant 1, k>m\}$

with the convention that we ignore any box from the "zeroth" row. We define $\operatorname{Gar}_{\triangleright}([r, c, m])$ to be the intersection of $\operatorname{Gar}_{\succ}([r, c, m])$ with the $m$ th component and refer to this as the $(\triangleright)$ Garnir belt. 
Example 2.7 Let $\sigma=(0,3,8) \in \mathbb{Z}^{3}$ and $e=14$. Given $\lambda=\left(\left(3^{2}, 2^{2}, 1\right),\left(5^{2}, 3,2,1\right),\left(4^{2}, 3,1^{2}\right)\right)$ and the box $[3,3,1] \in \lambda$, we colour $\operatorname{Gar}_{\succ}([3,3,1]) \cap \lambda$ below

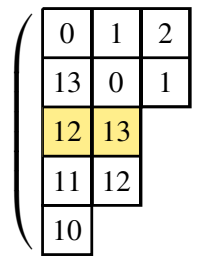

\begin{tabular}{|l|l|l|l|l|}
\hline 3 & 4 & 5 & 6 & 7 \\
\hline 2 & 3 & 4 & 5 & 6 \\
\hline 1 & 2 & 3 & \multicolumn{2}{|c}{} \\
\cline { 1 - 2 } 0 & 1 & \multicolumn{2}{|c}{} \\
\cline { 1 - 2 } 14 & \multicolumn{3}{|c}{} \\
\cline { 1 - 2 } & & &
\end{tabular}

\begin{tabular}{|c|c|c|c|}
\hline 8 & 9 & 10 & 11 \\
\cline { 1 - 3 } 7 & 8 & 9 & 10 \\
\cline { 1 - 2 } 6 & 7 & 8 & \multicolumn{1}{|c}{} \\
\cline { 1 - 2 } 5 & \multicolumn{3}{|c}{} \\
\cline { 1 - 1 } 4 & \multicolumn{2}{|c}{} \\
\cline { 1 - 1 } & & &
\end{tabular}

The $(\triangleright)$-Garnir belt is the subset of boxes coloured in orange.

Given $\underline{h} \in \mathbb{N}^{\ell}$, we let $\mathscr{P}_{\underline{h}}(n)$ (respectively $\left.\mathscr{C}_{\underline{h}}(n)\right)$ denote the subsets of $\ell$-multipartitions (respectively $\ell$-multicompositions) with at most $h_{m}$ columns in the $m$ th component.

Lemma 2.8 Let $\lambda \in \mathscr{P}_{\underline{h}}(n)$. For any box $[i, j, m]$, the multiset of residues of the boxes in $\lambda \cap \operatorname{Gar}_{\succ}[i, j, m]$ is multiplicity-free (i.e. no residue appears more than once).

Proof This follows immediately from the definitions since $\lambda \in \mathscr{P}_{\underline{h}}(n)$.

Let $\geqslant$ be an ordering on $\mathcal{B}_{\ell}(n)$. Given $1 \leqslant k \leqslant n$, we let $\mathcal{A}_{\mathrm{T}}^{\geqslant}(k)$, (respectively $\mathcal{R}_{\mathrm{T}}^{\geqslant}(k)$ ) denote the set of all addable res $\left(\mathrm{T}^{-1}(k)\right.$ )-boxes (respectively all removable $\operatorname{res}\left(\mathrm{T}^{-1}(k)\right)$ boxes) of the box-configuration Shape $\left(\mathrm{T} \downarrow_{\{1, \ldots, k\}}\right)$ which are less than $\mathrm{T}^{-1}(k)$ in the $\geqslant$-order. We define the $(\geqslant)$-degree of $\operatorname{T} \in \operatorname{Std}(\lambda)$ for $\lambda \in \mathscr{P}_{\ell}(n)$ as follows,

$$
\operatorname{deg}_{\geqslant}(\mathrm{T})=\sum_{k=1}^{n}\left(\left|\mathcal{A}_{\mathrm{T}}^{\geqslant}(k)\right|-\left|\mathcal{R}_{\mathrm{T}}(k)\right|\right) .
$$

We let $\operatorname{res}(\mathrm{T})$ denote the residue sequence consisting of $\operatorname{res}\left(\mathrm{T}^{-1}(k)\right)$ for $k=1, \ldots, n$ in order. We set $e_{\mathrm{T}}:=e_{\text {res( } \mathrm{T})} \in \mathscr{H}_{n}^{\sigma}$. We set

$$
y_{\lambda}^{\succ}=\prod_{k=1}^{n} y_{k}^{\left|\mathcal{A}_{\mathrm{T}_{\lambda}}^{\succ}(k)\right|} e_{\mathrm{T}_{\lambda}} \quad \text { and } \quad y_{\lambda}^{\triangleright}=\prod_{k=1}^{n} y_{k}^{\left|\mathcal{A}_{\mathrm{S}_{\lambda}}^{\triangleright}(k)\right|} e_{\mathrm{S}_{\lambda} .}
$$

For $\lambda \in \mathscr{P}_{\ell}(n)$, the element $y_{\lambda}^{\triangleright}$ was first defined in [7, Definition 4.15]. We remark that $y_{\lambda}^{\succ}=e_{\mathrm{T}_{\lambda}}$ for $\lambda \in \mathscr{P}_{\underline{h}}(n)$. Given $\mathrm{S}, \mathrm{T} \in \operatorname{Std}(\lambda)$ and $\underline{w}$ any fixed reduced word for $w_{\mathrm{T}}^{\mathrm{S}}$ we let $\psi_{\mathrm{T}}^{\mathrm{S}}:=e_{\mathrm{S}} \psi_{\underline{w}} e_{\mathrm{T}}$.

Definition 2.9 We set $\mathcal{Y}_{n}=\left\langle e_{\underline{i}}, y_{k} \mid \underline{i} \in(\mathbb{Z} / e \mathbb{Z})^{n}, 1 \leqslant k \leqslant n\right\rangle$.

\subsection{The quotient}

A long-standing belief in modular Lie theory is that we should (first) restrict our attention to fields whose characteristic, $p$, is greater than the Coxeter number, $h$, of the algebraic group we are studying. This allows one to consider a "regular block" of the algebraic group in question. What does this mean on the other side of the Schur-Weyl duality relating $\mathrm{GL}_{h}(\mathbb{k})$ and $\mathbb{k} \mathfrak{S}_{n}$ ? By the second fundamental theorem of invariant theory, the kernel of the group algebra of the symmetric group acting on $n$-fold $h$-dimensional tensor space is the 2 -sided ideal generated by the element

$$
\sum_{g \in \mathfrak{S}_{h+1} \leqslant \mathfrak{S}_{n}} \operatorname{sgn}(g) g \in \mathbb{k} \mathfrak{S}_{n}
$$


Modulo "more dominant terms" this element can be rewritten in the form we introduced in Eq. (2.2), as follows

$$
y_{(h+1)}^{\succ} \otimes 1_{\mathcal{H}_{n-h-1}^{\sigma}}
$$

by [7, 4.16 Corollary]. The simples of this algebra are indexed by partitions with at most $h$ columns. Given $\sigma \in \mathbb{Z}^{\ell}$, we let $\underline{h}=\left(h_{0}, \ldots, h_{\ell-1}\right) \in \mathbb{N}^{\ell}$ be such that $h_{m} \leqslant \sigma_{m+1}-\sigma_{m}$ for $0 \leqslant m<\ell-1$ and $h_{\ell-1}<e+\sigma_{0}-\sigma_{\ell-1}$. We define

$$
\mathrm{y}_{\underline{h}}=\sum_{0 \leqslant m<\ell} y_{\left(\emptyset, \ldots, \emptyset,\left(h_{m}+1\right), \emptyset, \ldots, \emptyset\right)}^{\succ} \otimes 1_{\mathcal{H}_{n-h_{m}-1}^{\sigma}},
$$

to be the higher level analogue of the element in (2.3).

Definition 2.10 Given weakly increasing $\sigma \in \mathbb{Z}^{\ell}$, we let $\underline{h}=\left(h_{0}, \ldots, h_{\ell-1}\right) \in \mathbb{N}^{\ell}$ be such that $h_{m} \leqslant \sigma_{m+1}-\sigma_{m}$ for $0 \leqslant m<\ell-1$ and $h_{\ell-1}<e+\sigma_{0}-\sigma_{\ell-1}$. We define $\mathscr{H}_{n}^{\sigma}:=\mathcal{H}_{n}^{\sigma} / \mathcal{H}_{n}^{\sigma} \mathrm{y}_{\underline{h}} \mathcal{H}_{n}^{\sigma}$.

In level $\ell=1$, the condition of Definition 2.10 is equivalent to $h_{0}<e$ (and $\mathscr{P}_{\underline{h}}(n)$ is the set of partitions of $n$ with strictly fewer than $e$ columns).

\subsection{Generator/partition combinatorics}

Our cellular basis will provide a stratification of $\mathscr{H}_{n}^{\sigma}$ in which each layer is generated by an idempotent correspond to some multipartition. Whence we wish to understand the effect of multiplying a generator of a given layer in the cell-stratification by a KLR "dot generator". This leads us to define combinatorial analogues of the dot generators as maps on the set of box configurations.

Definition 2.11 Let $\lambda \in \mathcal{B}_{\ell}(n)$ and let $[i, j, m] \in \lambda$. We say that $[i, j, m]$ is left-justified if either $j \leqslant e$ or there exists some $[i, j-p, m] \in \lambda$ for $1 \leqslant p \leqslant e$.

Definition 2.12 Let $\lambda \in \mathcal{B}_{\ell}(n)$. For $\alpha \in \lambda$ an $r$-box, we define $Y_{\alpha}(\lambda)=\lambda-\alpha \cup \beta$ where the $r$-box $\beta \notin \lambda$ is such that $\beta \succ \alpha$, it is left-justified, and is minimal in $\succ$ with respect to these properties (if such a box exists). If such a box does not exist then we say that $Y_{\alpha}(\lambda)$ and $\beta$ are both undefined.

We write $\lambda \gg \mu$ if $\lambda=Y_{\alpha}(\mu)$ for some $\alpha \in \mu$ and we then extend $\gg$ to a partial ordering on $\mathcal{B}_{\ell}(n)$ by taking the transitive closure. Suppose that $\left\{\left[i_{k}, j_{k}, m_{k}\right] \mid 0<k \leqslant p\right\}$ is a set of $r$-boxes and that $Y_{\left[i_{k}, j_{k}, m_{k}\right]}\left(\lambda \cup\left[i_{k}, j_{k}, m_{k}\right]\right)=\lambda \cup\left[i_{k+1}, j_{k+1}, m_{k+1}\right]$ for $k \geqslant 1$. We define

$$
Y_{\left[i_{1}, j_{1}, m_{1}\right]}^{p}\left(\lambda \cup\left[i_{1}, j_{1}, m_{1}\right]\right)=\left(\lambda \cup\left[i_{p}, j_{p}, m_{p}\right]\right) .
$$

We remark that $\lambda \succcurlyeq \mu$ implies that $\lambda \succ \mu$ and so $\succcurlyeq$ is a coarsening of $\succ$.

Example 2.13 Let $e=5$ and $\ell=1$. For $\lambda=\left(3,2^{2}, 1^{6}\right) \in \mathscr{P}_{1}(13)$, we have that $Y_{[3,2,0]}(\lambda)=$ $\left(3,2,1^{7}\right) \cup[2,6,0]$. We have that $Y_{[3,2,0]}^{2}(\lambda)=Y_{[2,6,0]}\left(\left(3,2,1^{7}\right) \cup[2,6,0]\right)=\left(3,2,1^{7}\right) \cup$ $[1,5,0]$.

Example 2.14 Let $e=5$ and $\ell=1$ and $r=2$. For $\lambda=\left(3,2^{2}, 1^{6}\right) \in \mathscr{P}_{1}(13)$, we have $Y_{[4,1,0]}^{1}(\lambda)=\left(3,2^{2}, 1^{6}\right) \cup[3,5,0]-[4,1,0]$ and $Y_{[4,1,0]}^{2}(\lambda)=\left(3,2^{2}, 1^{6}\right) \cup[2,4,0]-[4,1,0]$ (see Fig. 2). 

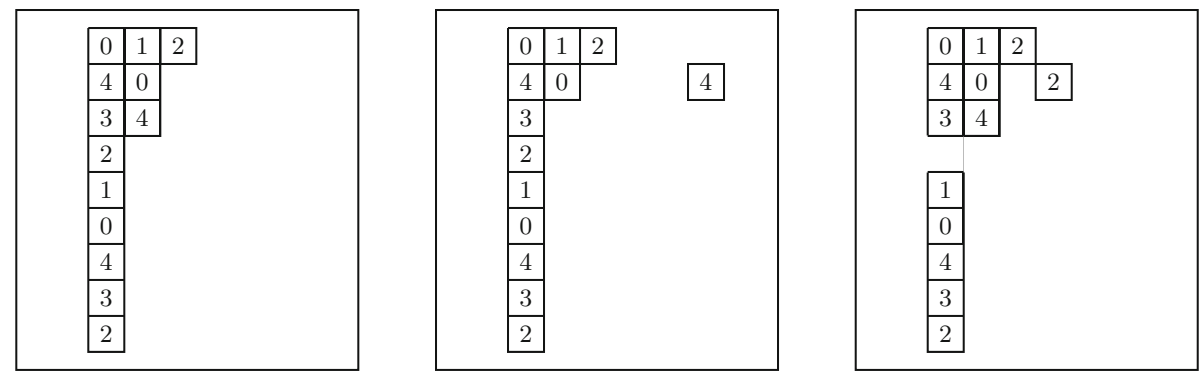

Fig. 2 The box configurations, $\lambda, Y_{[3,2,0]}(\lambda)$, and and $Y_{[4,1,0]}^{2}(\lambda)$

Given an idempotent generator, $e_{j}$ for $j \in(\mathbb{Z} / e \mathbb{Z})^{n}$, of the KLR algebra, we wish to identify to which layer of our stratification our idempotent belongs. To this end we make the following definition.

Definition 2.15 Associated to any $\underline{j}=\left(j_{1}, \ldots, j_{n}\right) \in(\mathbb{Z} / e \mathbb{Z})^{n}$, we have an element $\mathrm{J} \in$ $\operatorname{Std}(n) \cup\{0\}$ given by placing the entry $k=1,2, \ldots, n+1$ in the lowest addable $j_{k}$-box under $\succ$ of the partition Shape $\left(J_{\leqslant k-1}\right)$, and formally setting $J=0$ and Shape $(J)=0$ if no such box exists for some $1 \leqslant k \leqslant n$.

\subsection{A tableaux theoretic basis}

We are now ready to construct our first basis of $\mathscr{H}_{n}^{\sigma}$. This basis will serve as the starting point for our light-leaves bases of Theorem A. The combinatorics of this basis will be familiar to anyone who has studied symmetric groups and cyclotomic Hecke algebras (but with respect to the, less familiar, $(\succ)$-ordering).

Definition 2.16 Let $\lambda \in \mathscr{P}_{\underline{h}}(n)$. We define the Garnir adjacency set of an $r$-box $\alpha=$ $[i, j, m] \notin \lambda$ to be the set of boxes, $\gamma \in \lambda \cap \operatorname{Gar}_{\succ}(\alpha)$ such that $|\operatorname{res}(\gamma)-r| \leqslant 1$ and denote this set by $\operatorname{Adj-Gar}_{\succ}(\alpha)$. We set $\operatorname{res}\left(\operatorname{Adj}_{-G}(\alpha)\right)=\left\{\operatorname{res}(\gamma) \mid \gamma \in \operatorname{Adj}_{\succ}-\operatorname{Gar}_{\succ}(\alpha)\right\}$.

Example 2.17 Continuing with Example 2.7, we have that $\operatorname{Adj-Gar}_{\succ}([3,3,1])=\{[3,2,1]$, $[2,3,1]\}$.

Remark 2.18 We are endeavouring to construct a 2-sided chain of ideals of $\mathscr{H}_{n}^{\sigma}$, ordered by », in which each 2-sided ideal is generated by an idempotent $e_{T_{\lambda}}$ for $\lambda \in \mathscr{P}_{\underline{h}}(n)$. Equations (2.6) and (2.7) of Theorem 2.19 will allow us to rewrite any element of $\mathcal{Y}_{n}$ in the required form by moving a given box $\square$ through the partition $\lambda$ one row at a time along the $\succ$ ordering until it comes to rest at some point $\lambda \cup \square^{\prime} \in \mathscr{P}_{\underline{h}}(n)$. For $\mathrm{J}$ the tableau in Fig. 3, then the eight steps involved in rewriting $e_{\mathrm{J}}$ as an element of $\mathscr{H}^{\succeq\left(3,2^{2}, 1^{6}\right)}$ are illustrated in Fig. 4.

The following theorem is the technical heart of this section. We define $\mathscr{H}^{\geqslant \lambda}=\mathscr{H}_{n}^{\sigma}\left\langle e_{\mathrm{T}_{v}}\right|$ $v \geqslant \lambda\rangle \mathscr{H}_{n}^{\sigma}$ (respectively $\mathcal{H}^{\geqslant \lambda}=\mathcal{H}_{n}^{\sigma}\left\langle e_{\mathrm{T}_{v}} \mid v \geqslant \lambda\right\rangle \mathcal{H}_{n}^{\sigma}$ ) for $\geqslant$ any ordering on $\mathscr{P}_{h}(n)$ (respectively $\left.\mathscr{P}_{\ell}(n)\right)$. Given $\geqslant$ a total order on $\mathscr{P}_{\underline{h}}(n)$, we let $\lambda^{[0]}>\lambda^{[1]}>\cdots>\lambda^{[m]}$ denote the complete set of elements of $\mathscr{P}_{\underline{h}}(n)$ enumerated according to the total ordering $>$. Given $\lambda \in \mathcal{B}_{\ell}(n-1)$ and $\alpha \notin \lambda$, we define

$$
\mathscr{H}_{n}^{(\succcurlyeq \lambda) \cup \alpha}=\sum_{\left\{\mu \in \mathcal{B}_{\ell}(n-1) \mid \mu \gg \lambda, \alpha \notin \mu\right\}} \mathscr{H}_{n}^{\sigma} e_{\mathrm{\top}_{\mu \cup \alpha}} \mathscr{H}_{n}^{\sigma} \leqslant \mathscr{H}_{n}^{\gg(\lambda \cup \alpha)} .
$$



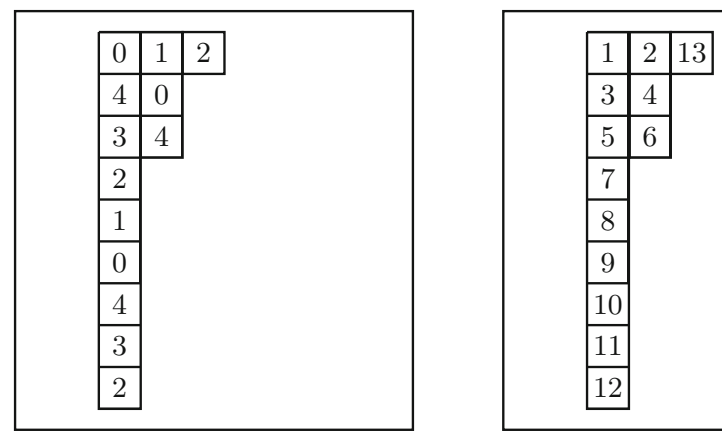

Fig. 3 Let $e=5$ and $\ell=1$. On the left, we have the residues for the partition $\lambda=\left(3,2^{2}, 1^{6}\right)$. On the right, we have the tableau $\mathrm{J} \in \operatorname{Std}\left(3,2^{2}, 1^{6}\right)$ for $\underline{j}=(0,1,4,0,3,4,2,1,0,4,3,2,2)$ as in Definition 2.15

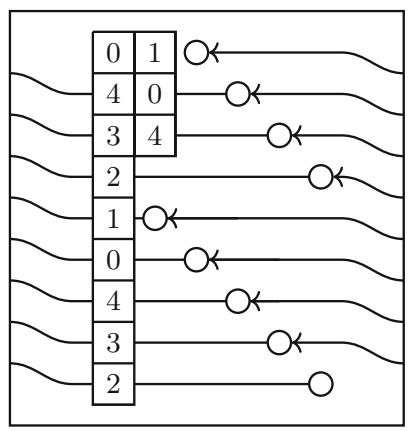

Fig. 4 For $n=13$ and $\lambda=\left(2^{3}, 1^{6}\right)$, we illustrate how the idempotent, $e_{(0,1,4,0,3,4,2,1,0,4,3,2,2)}$, labelled by $\mathrm{J}$ in Fig. 3 is rewritten in the form (2.8). The box moves through each row until it comes to rest at the point $\mathrm{J}^{-1}(13)=[1,3,0]$. This involves repeated applications of Eq. (2.6) to deduce (2.8). For the purposes of later referencing, we label the 9 boxes from bottom-to-top by $\alpha_{1}=[9,6,0], \alpha_{2}=[8,5,0], \ldots, \alpha_{9}=[1,3,0]$. This visualisation is explained in Remark 2.18

Theorem 2.19 Let $\lambda \in \mathscr{P}_{\underline{h}}(n-1)$ and assume $\alpha=[i, j, m] \notin \lambda$ is left-justified. We set $a=\mathrm{T}_{\lambda \cup \alpha}(\alpha)$.

(a) If $\alpha=[1, j, 0]$ for some $j \geqslant 1$ then (i) $y_{\mathrm{T}_{\lambda \cup \alpha}}=0$ if $\lambda \cup \alpha \notin \mathscr{P}_{\underline{h}}(n)$ and (ii) $y_{a} y_{\mathrm{T}_{\lambda \cup \alpha}}=0$ if $\lambda \cup \alpha \in \mathscr{P}_{\underline{h}}(n)$.

(b) For $\alpha \neq[1, \bar{j}, 0]$ for some $j \geqslant 1$, we set $\beta$ to be the box determined by

$$
\begin{cases}Y_{\alpha}^{\ell+1}(\lambda \cup \alpha)=\lambda \cup \beta & \text { if } \operatorname{res}(\operatorname{Adj}-\operatorname{Gar}(\alpha))=\{r-1\} ; \\ Y_{\alpha}^{1}(\lambda \cup \alpha)=\lambda \cup \beta & \text { otherwise }\end{cases}
$$

and we set $b=\mathrm{T}_{\lambda \cup \beta}(\beta)$.

- If $\lambda \cup \alpha \notin \mathscr{P}_{\underline{h}}(n)$, then we have that

$$
y_{\mathrm{T}_{\lambda \cup \alpha}}^{\succ} \in\left\{\begin{array}{l} 
\pm \psi_{b}^{a} y_{\mathrm{T}_{\lambda \cup \beta}}^{\succ} \psi_{a}^{b}+\mathscr{H}_{n}^{(\succcurlyeq \lambda) \cup \beta} \\
\pm\left(y_{a-1} \psi_{b}^{a} y_{\mathrm{T}_{\lambda \cup \beta}}^{\succ} \psi_{a}^{b}-\psi_{b}^{a} y_{\mathrm{T}_{\lambda \cup \beta}}^{\succ} \psi_{a}^{b} y_{a}\right)+\mathscr{H}_{n}^{(\succcurlyeq \lambda) \cup \beta}
\end{array}\right. \text { or }
$$

(the cases are detailed in the proof). 


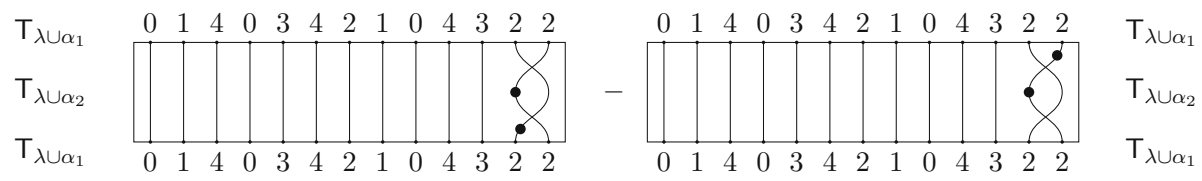

Fig. 5 We continue with the example in Fig. 4 for $\lambda=\left(2^{6}, 1^{3}\right)$. This is the righthand-side of Eq. (2.10) for $e_{\mathrm{T}_{\lambda \cup \alpha_{1}}}$

- If $\lambda \cup \alpha \in \mathscr{P}_{\underline{h}}(n)$, then we have that

$$
y_{a}\left(y_{\mathrm{T}_{\lambda \cup \alpha}}^{\succ}\right) \in \pm \psi_{b}^{a} y_{\mathrm{\top}_{\lambda \cup \beta}}^{\succ} \psi_{a}^{b}+\mathscr{H}_{n}^{(\succcurlyeq \lambda) \cup \beta}
$$

(c) For $j \in(\mathbb{Z} / e \mathbb{Z})^{n}$, using the notation of Definition 2.15, we have that:

$$
\begin{cases}e_{\underline{j}} \in \pm \tilde{\psi}_{\mathrm{T}_{v}}^{J} \tilde{\psi}_{\jmath}^{T_{v}}+\mathscr{H}^{\gg v} & \text { if } \operatorname{Shape}(J)=v \in \mathscr{P}_{\underline{h}}(n) \\ e_{\underline{j}}=0 & \text { if } \operatorname{Shape}(J)=0\end{cases}
$$

where $\tilde{\psi}_{\mathrm{T}_{v}}^{J}$ is obtained from $\psi_{\mathrm{T}_{v}}^{J}$ by possibly adding some dot decorations along the strands. Thus if $\geqslant$ is any total refinement of $\gg$ then the $\mathbb{Z}$-algebra $\mathscr{H}_{n}^{\sigma}$ has a chain of two-sided ideals

$$
0 \subset \mathscr{H}_{n}{ }^{[0]} \subset \mathscr{H}_{n}{ }^{[1]} \subset \cdots \subset \mathscr{H}_{n} \lambda^{[m]}=\mathscr{H}_{n}^{\sigma} .
$$

Remark 2.20 In the proof, we will often relate ideals in smaller and larger algebras using horizontal concatenation of diagrams, this is made possible by the definition of the reverse cylindric ordering $\succ$ (which distinguishes between box configurations based on the first discrepancy upon reading a pair of box configurations backwards).

Remark 2.21 If $\lambda \cup \alpha \in \mathscr{P}_{\underline{h}}(n)$, then $y_{\top_{\lambda \cup \alpha}}^{\succ}=e_{T_{\lambda \cup \alpha}}$ by Lemma 2.8.

Proof of Theorem 2.19 Part (a), let $\alpha=[1, j, 0]$ for some $j \geqslant 1$. Claim (ii) follows by applying case 3 of R $4 a$ times, followed by the commuting KLR relations and the cyclotomic relation. The proof of claim (i) is similar. Thus (a) follows.

For parts (b) and (c), we assume that Eqs. (2.6)-(2.9) all hold for rank $n-1$. We further assume that Eqs. (2.7) and (2.6) have been proven for all $v=\mu \cup \alpha$ with $\mu \in \mathscr{P}_{\underline{h}}(n-1)$ such that $\mu \succcurlyeq \lambda$; thus leaving us to prove Eqs. (2.7) and (2.6) for $v=\lambda \cup \alpha$ for $\lambda \in \overline{\mathscr{P}}_{\underline{h}}(n-1)$ and Eq. (2.8) for all $j \in(\mathbb{Z} / e \mathbb{Z})^{n}$. Equation (2.9) follows immediately from Eq. (2.8) and the idempotent decomposition of the identity in relation R1. By Definition 2.12, $\operatorname{res}(\alpha)=\operatorname{res}(\beta)$ and we set this residue equal to $r \in \mathbb{Z} / e \mathbb{Z}$.

Proof of Eq. (2.6) for a given $\lambda$ and $\alpha$. We include a running example for $e=5$ and $\ell=1$ and $\lambda=\left(2^{3}, 1^{6}\right)$. We assume that $\lambda \cup \alpha \notin \mathscr{P}_{\underline{h}}(n)$. There are four cases to consider, depending on the residue of $\alpha^{\prime}:=\mathrm{T}_{\lambda \cup \alpha}^{-1}(a-1)$. We assume that $\alpha^{\prime}$ is in the same row of the same component as $\alpha$ (as otherwise $y_{\mathrm{T}_{\lambda \cup \alpha}}=y_{\mathrm{T}_{\lambda \cup \beta}}$ by definition). We let $\beta^{\prime}$ be the box determined by

$$
\begin{cases}Y_{\alpha^{\prime}}^{\ell+1}\left(\lambda \cup \alpha^{\prime}\right)=\lambda \cup \beta^{\prime} & \text { if } \operatorname{res}\left(\operatorname{Adj}-\operatorname{Gar}\left(\alpha^{\prime}\right)\right)=\{r-1\} \\ Y_{\alpha^{\prime}}^{1}\left(\lambda \cup \alpha^{\prime}\right)=\lambda \cup \beta^{\prime} & \text { otherwise. }\end{cases}
$$


(i) Suppose $\alpha^{\prime}$ has residue $r \in \mathbb{Z} / e \mathbb{Z}$ and so $y_{\mathrm{T}_{\lambda \cup \alpha}}^{\succ}=e_{\mathrm{T}_{\lambda \cup \alpha}}$ by Lemma 2.8. By application of relations $\mathrm{R} 3$ and $\mathrm{R} 4$, we have that

$$
e_{\mathrm{T}_{\lambda \cup \alpha}}=\psi_{a-1} y_{a-1} e_{\mathrm{T}_{\lambda \cup \alpha}} \psi_{a} y_{a-1}-y_{a} \psi_{a-1} y_{a-1} e_{\mathrm{T}_{\lambda \cup \alpha}} \psi_{a-1} \text {. }
$$

An example of the visualisation of the idempotents on the righthand-side of Eq. (2.10) is given in the first step of Fig. 4; the corresponding righthand-side of Eq. (2.10) is depicted in Fig. 5. Now, we have that

$$
y_{a-1} e_{\mathrm{T}_{\lambda \cup \alpha}}=\left(y_{a-1} e_{\mathrm{T}_{\lambda \cup \alpha \downarrow \leqslant a-1}}\right) \otimes e_{r} \otimes e_{\mathrm{T}_{\lambda \cup \alpha} \downarrow>a}
$$

and so by our inductive assumption for Eq. (2.7) for rank $a-1<n$, we have that

$$
\begin{gathered}
y_{a-1} e_{\mathrm{T}_{\lambda \cup \alpha}} \in \pm \psi_{b}^{a-1} y_{\mathrm{T}_{\lambda \cup \beta \downarrow}}^{\succ}{ }_{a-1} \psi_{a-1}^{b} \otimes e_{\mathrm{T}_{\lambda \cup \beta} \downarrow \geqslant a}+\mathscr{H}_{n}^{(\succcurlyeq \lambda) \cup \beta} \\
= \pm \psi_{b}^{a-1} y_{\mathrm{\top}_{\lambda \cup \beta}}^{\succ} \psi_{a-1}^{b}+\mathscr{H}_{n}^{(\succcurlyeq \lambda) \cup \beta}
\end{gathered}
$$

where we have implicitly used the following facts: (i) $Y_{\alpha^{\prime}}(\lambda \cup \alpha)=\lambda \cup \alpha \cup \beta-\alpha^{\prime}$ (ii) $\mathrm{T}_{\lambda \cup \alpha \downarrow>a}=\mathrm{T}_{\lambda \cup \beta \downarrow} \downarrow_{>a}$ and (iii) $e_{\mathrm{T}_{\lambda \cup \beta \cup \alpha-\alpha^{\prime}}}=e_{\mathrm{T}_{\lambda \cup \beta}}$. Substituting this back into Eq. (2.10), we obtain

$$
e_{\mathrm{T}_{\lambda \cup \alpha}} \in \pm\left(\psi_{b}^{a} y_{\mathrm{T}_{\lambda \cup \beta}}^{\succ} \psi_{a}^{b} y_{a-1}-y_{a} \psi_{b}^{a} y_{\mathrm{T}_{\lambda \cup \beta}}^{\succ} \psi_{a}^{b}\right)+\mathscr{H}_{n}^{(\succcurlyeq \lambda) \cup \beta}
$$

as required (as in the second case in Eq. (2.6)). An example is depicted in Fig. 5 (although we remark that the error terms belonging to $\mathscr{H}_{n}^{(\succcurlyeq \lambda) \cup \beta}$ are actually all zero in this case).

(ii) Now suppose $\alpha^{\prime}=[x, y, z]$ has residue $r+1 \in \mathbb{Z} / e \mathbb{Z}$. We have two subcases to consider. We first consider the easier subcase, in which $[x, y, z]=[i, 1, m]$ and so $b=a-1$. We have that $[i+1,1, m] \in \operatorname{Add}_{r}\left(\mathrm{~T}_{\lambda \cup \alpha} \downarrow \leqslant a\right)$ whereas $\operatorname{Add}_{r}\left(\mathrm{~T}_{\lambda \cup \beta \downarrow} \downarrow b\right)=\emptyset$. By relation $\mathrm{R} 4$, we have that

$y_{\mathrm{T}_{\lambda \cup \alpha}}^{\succ}=y_{a} e_{\mathrm{T}_{\lambda \cup \alpha}}=y_{a-1} e_{\mathrm{T}_{\lambda \cup \alpha}}-e_{\mathrm{T}_{\lambda \cup \alpha}} \psi_{a-1} e_{\mathrm{T}_{\lambda \cup \beta}} \psi_{a-1} e_{\mathrm{T}_{\lambda \cup \alpha}}=y_{a-1} e_{\mathrm{T}_{\lambda \cup \alpha}}-\psi_{b}^{a} y_{\mathrm{T}_{\lambda \cup \beta}}^{\succ} \psi_{a}^{b}$.

We have that $y_{a-1} y_{T_{\lambda}} \in \mathscr{H}_{n-1}^{\Downarrow \lambda \cup \beta^{\prime}-\alpha^{\prime}}$ and so the former term is of the required form by our assumption for $\lambda \cup \beta^{\prime}-\alpha^{\prime}=\mu \gg \lambda$.

Now, if $y>1$ then the $(a-2)$ th, $(a-1)$ th and $a$ th strands have residues $r, r+1$, and $r$ respectively. We have that

$$
\begin{aligned}
y_{\mathrm{T}_{\lambda \cup \alpha}}^{\succ}=e_{\mathrm{T}_{\lambda \cup \alpha}} & =e_{\mathrm{T}_{\lambda \cup \alpha}} \psi_{a-2} \psi_{a-1} \psi_{a-2} e_{\mathrm{T}_{\lambda \cup \alpha}}-e_{\mathrm{T}_{\lambda \cup \alpha}} \psi_{a-1} \psi_{a-2} \psi_{a-1} e_{\mathrm{T}_{\lambda \cup \alpha}} \\
& =-e_{\mathrm{T}_{\lambda \cup \alpha}} \psi_{a-2} \psi_{a-1} y_{a-1} \psi_{a-1} \psi_{a-2} e_{\mathrm{T}_{\lambda \cup \alpha}}+e_{\mathrm{T}_{\lambda \cup \alpha}} \psi_{a-1} \psi_{a-2} y_{a-2} \psi_{a-2} \psi_{a-1} e_{\mathrm{T}_{\lambda \cup \alpha}} .
\end{aligned}
$$

where the first equality follows from Lemma 2.8, the second from relation R5 and the third follows from relations R3 and R4. We set $\xi=\operatorname{Shape}\left(\mathrm{T}_{\lambda \downarrow} \downarrow_{<a-2}\right)$. The two terms in Eq. (2.12) factor through the elements

$$
\underbrace{e_{\mathrm{T}_{\lambda \downarrow}<a-2} \otimes e_{r+1}}_{\xi \cup[x, y, z]} \nabla y_{1} e_{r, r} \otimes e_{\mathrm{T}_{\lambda \cup \alpha \downarrow} \downarrow a} \quad \underbrace{e_{\mathrm{T}_{\lambda \downarrow<a-2}} \otimes y_{1} e_{r}}_{\xi \cup[x, y-1, z]} \nabla e_{r, r+1} \otimes e_{\mathrm{T}_{\lambda \cup \alpha \downarrow} \downarrow a}
$$

respectively.

- We first consider the latter term on the righthand-side of Eq. (2.12) (which we will see, is the required non-zero term). We note that $[x, y-1, z]$ and $\alpha$ have the same residue 
and so $Y_{[x, y-1, z]}(\xi \cup[x, y-1, z])=\xi \cup \beta$. By our inductive assumption that Eq. (2.7) holds for rank $a-2<n$, we have that

$$
e_{\mathrm{T}_{\lambda \downarrow<a-2}} \otimes y_{1} e_{r}=y_{a-2} e_{\mathrm{T}_{\xi \cup[x, y-1, z]}} \in \pm \psi_{b}^{a-2} y_{\mathrm{T}_{\xi \cup \beta}^{\succ}}^{\succ} \psi_{a-2}^{b}+\mathscr{H}_{a-2}^{(\succcurlyeq \xi) \cup \beta}
$$

Substituting this back into the second term of Eq. (2.13) we obtain

$$
\psi_{b}^{a-2} y_{\mathrm{\top}_{\xi \cup \beta}}^{\succ} \psi_{a-2}^{b} \otimes e_{r, r+1} \otimes e_{\mathrm{T}_{\lambda \cup \alpha} \downarrow>a} \in \pm \psi_{b}^{a-2} y_{\mathrm{T}_{\lambda \cup \beta}}^{\succ} \psi_{a-2}^{b}+\mathscr{H}_{n}^{(\succcurlyeq \lambda) \cup \beta}
$$

and then substituting into the second term of Eq. (2.12) we obtain

$$
\begin{aligned}
e_{\mathrm{T}_{\lambda \cup \alpha}} \psi_{a-1} \psi_{a-2} y_{a-2} \psi_{a-2} \psi_{a-1} e_{\mathrm{T}_{\lambda \cup \alpha}} & \in \pm \psi_{a-1} \psi_{a-2} \psi_{b}^{a-2} y_{\mathrm{T}_{\lambda \cup \beta}}^{\succ} \psi_{a-2}^{b} \psi_{a-2} \psi_{a-1}+\mathscr{H}_{n}^{(\succcurlyeq \lambda) \cup \beta} \\
& = \pm \psi_{b}^{a} y_{\mathrm{T}_{\lambda \cup \beta}}^{\succ} \psi_{a}^{b}+\mathscr{H}_{n}^{(\gg \lambda) \cup \beta}
\end{aligned}
$$

as required.

- We now consider the former term of Eq. (2.12) (which, we will see, is zero modulo the ideal). We have that $Y_{[x, y, z]}(\xi \cup[x, y, z])=(\xi \cup \gamma) \gg(\xi \cup[x, y, z])$ for $\gamma$ a box of residue $r+1 \in \mathbb{Z} / e \mathbb{Z}$. We set $c=\mathrm{T}_{\xi \cup \gamma}^{-1}(\gamma)$. We have that

$$
e_{\mathrm{T}_{\xi}} \otimes e_{r+1}=e_{\mathrm{T}_{\xi \cup[x, y, z]}} \in \pm \psi_{c}^{a-2} y_{\mathrm{T}_{\xi \cup \gamma}}^{\succ} \psi_{a-2}^{c}+\mathscr{H}_{a-2}^{(\succcurlyeq \xi) \cup \gamma}
$$

by our inductive assumption that Eq. (2.6) holds for rank $a-2<n$. Concatenating, we have that

$e_{\mathrm{T}_{\lambda \downarrow<a-2}} \otimes e_{r+1} \otimes y_{1} e_{r, r} \otimes e_{\mathrm{T}_{\lambda \cup \alpha \downarrow}>a} \in \pm \psi_{c}^{a-2} y_{\mathrm{T}_{\lambda \cup \gamma \cup \alpha-[x, y, z]}}^{\succ} \psi_{a-2}^{c}+\mathscr{H}_{n}^{(\succcurlyeq(\lambda-[x, y, z] \cup \gamma)) \cup \alpha}$ and we note that the idempotent on the righthand-side belongs to the ideal $\mathscr{H}_{n}^{(\succcurlyeq(\lambda-[x, y, z] \cup \gamma)) \cup \alpha}$ and so the result follows by our assumption for $\lambda \cup \gamma-[x, y, z]=$ $\mu \gg \lambda$.

(iii) Now suppose $\alpha^{\prime}$ has residue $d \in \mathbb{Z} / e \mathbb{Z}$ such that $|d-r|>1$. We set $\xi=$ Shape $\left(\mathrm{T}_{\lambda \downarrow} \downarrow_{<-1}\right)$. By case 2 of relation $\mathrm{R} 4$, we have that

$$
y_{a}^{k} e_{\mathrm{T}_{\lambda \cup \alpha}}=\psi_{a-1}(\underbrace{e_{\mathrm{T}_{\lambda \downarrow}<a-1} \otimes y_{1}^{k} e_{r}}_{\xi \cup \alpha} \nabla e_{d} \otimes e_{\mathrm{T}_{\lambda \cup \alpha \downarrow>a}}) \psi_{a-1}
$$

for $k \in\{0,1\}$. By the inductive assumption for rank $a-1<n$ of Eq. (2.6), we have that

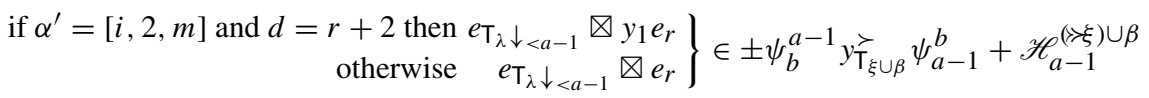

As in the case (ii) above, we concatenate to deduce the result. Two examples of the visualisation of the righthand-side of Eq. (2.15) are given in the third and fourth steps of Fig. 4; the corresponding elements are depicted in Fig. 6.

(iv) Suppose $\alpha^{\prime}=[x, y, z]$ has residue $r-1 \in \mathbb{Z} / e \mathbb{Z}$ (thus $[x, y, z]=[i, j-1, m]$ by residue considerations) and that $[i-1, j, m] \notin \lambda$ (if $[i-1, j, m] \in \lambda$, this implies that $\lambda \cup \alpha \in \mathscr{P}_{\underline{h}}(n)$ and so the process would terminate). We remark that $\operatorname{res}(\operatorname{Adj}-\operatorname{Gar}(\alpha))=$ $\{r-1\}$ and this is the unique case of the proof for which this holds.

Let $\gamma=[i-1, j-1, m]$ and we set $c=\mathrm{T}_{\lambda \cup \alpha}(\gamma)$ and let $\xi=\operatorname{Shape}\left(\mathrm{T}_{\lambda \downarrow}{ }_{<a-1}\right)$ (see Fig. 7 for an example). By Lemma 2.8, $e_{T_{\lambda \cup \alpha}}=y_{\mathrm{T}_{\lambda \cup \alpha}}^{\succ}$. We have that

$$
\begin{gathered}
e_{\mathrm{T}_{\lambda \cup \alpha}}=e_{\mathrm{T}_{\lambda \cup \alpha}} \psi_{a-2}^{c} \psi_{c}^{a-2} e_{\mathrm{T}_{\lambda \cup \alpha}} \\
=e_{\mathrm{T}_{\lambda \cup \alpha}} \psi_{a-1} \psi_{a-1}^{c} \psi_{c}^{a-2} \psi_{a-1} e_{\mathrm{T}_{\lambda \cup \alpha}}-e_{\mathrm{T}_{\lambda \cup \alpha}} \psi_{a-1}^{c} \psi_{c}^{a} e_{\mathrm{T}_{\lambda \cup \alpha}}
\end{gathered}
$$



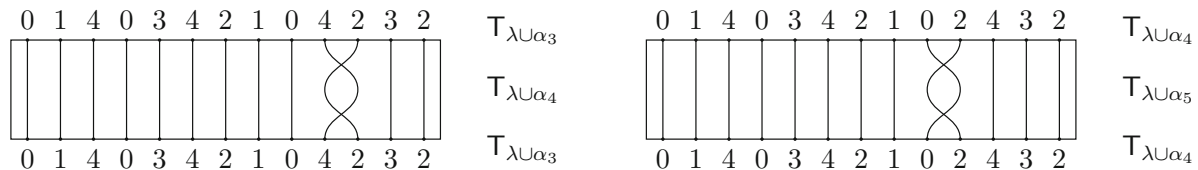

Fig. 6 The righthand-side of Eq. (2.15) for $\lambda=\left(2^{6}, 1^{3}\right)$ and $\alpha=\alpha_{3}$ and $\alpha_{4}$ respectively
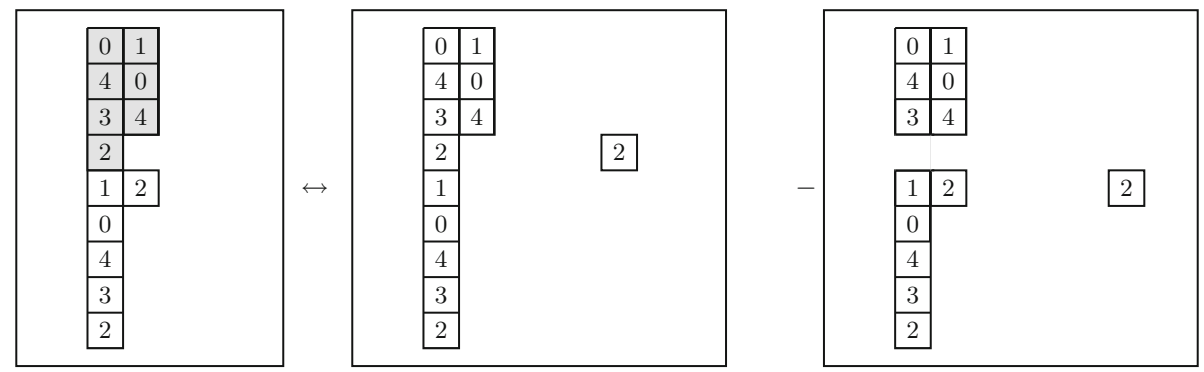

Fig. 7 The lefthand-side is $\lambda \cup \alpha_{5}$ as in Fig. 4 (with $\xi$ shaded grey). The righthand-side labels the idempotents obtained from applying Eq. (2.18). The middle box configuration is $T_{\lambda \cup \alpha_{6}}$

$$
\begin{gathered}
=e_{\mathrm{T}_{\lambda \cup \alpha}} \psi_{c+1}^{a} \psi_{a}^{c} e_{\mathrm{T}_{\lambda \cup \alpha}}-\psi_{a-1}^{c}\left(e_{\mathrm{T}_{\xi-\gamma}} \otimes e_{r-1, r, r} \otimes e_{\mathrm{T}_{\lambda \cup \alpha} \downarrow>a}\right) \psi_{c}^{a} \\
=-e_{\mathrm{T}_{\lambda \cup \alpha}} \psi_{c}^{a} y_{c} \psi_{a}^{c} e_{\mathrm{T}_{\lambda \cup \alpha}}-\psi_{a-1}^{c}\left(e_{\mathrm{T}_{\xi-\gamma}} \otimes e_{r-1, r, r} \otimes e_{\mathrm{T}_{\lambda \cup \alpha} \downarrow>a}\right) \psi_{c}^{a} \\
\in-\psi_{b}^{a} y_{\mathrm{T}_{\lambda \cup \beta}}^{\succ} \psi_{a}^{b} e_{\mathrm{T}_{\lambda \cup \alpha}}-\psi_{a-1}^{c}\left(e_{\mathrm{T}_{\xi-\gamma}} \otimes e_{r-1, r, r} \otimes e_{\mathrm{T}_{\lambda \cup \alpha \downarrow} \downarrow a}\right) \psi_{c}^{a}+\mathscr{H}^{(\succcurlyeq \lambda) \cup \beta}
\end{gathered}
$$

where the first and third equalities follow from the commuting case 2 of relation R4 and Lemma 2.8, and the second equality follows from case 1 of relation R5; the fourth equality follows from R3; the fifth is either trivial or follows from case (3) of R4 (in the latter case, the error term is zero by our inductive assumption for rank $c-1<n$ of Eq. (2.7)). For our continuing example, the righthand-side of Eq. (2.19) is depicted in Fig. 9; the box-configurations labelling the idempotents on the left and righthand-sides of Eq. (2.18) are depicted in Fig. 7.

We now consider the second term on the righthand-side of Eq. (2.18). We have that

$$
e_{\mathrm{T}_{\xi-\gamma}} \otimes e_{r-1, r, r}=e_{\mathrm{\top}_{\xi-\gamma \cup[i, j-1, m] \cup \alpha \cup[i, j+e, m]}}
$$

By our inductive assumption for ranks $a-2, a-1<n$ for Eq. (2.6), we have that:

$$
e_{\top_{\xi-\gamma \cup[i, j-1, m]}} \in \mathscr{H}_{a-2}^{\Downarrow \rho \rho} \Longrightarrow e_{\top_{\xi-\gamma \cup[i, j-1, m] \cup \alpha}} \in \mathscr{H}_{a-1}^{(\succeq \rho) \cup \gamma} \leqslant \mathscr{H}_{a-1}^{\Downarrow(\rho \cup \gamma)}
$$

for $\rho=Y_{[i, j-1, m]}^{\ell+1}(\xi-\gamma \cup[i, j-1, m])$. Given $\pi \succeq(\rho \cup \gamma)$, we can left justify $\pi \cup[i, j+e, m]$ to obtain $\pi \cup \alpha$. We note that $\operatorname{Gar}_{\succ}(\alpha) \cap \pi$ contains no nodes of residue $r$ or $r \pm 1$. Therefore

$$
e_{T_{\xi-\gamma \cup[i, j-1, m] \cup \alpha \cup[i, j+e, m]}} \in \mathscr{H}_{a}^{(\succeq \rho \cup \gamma) \cup \beta} \Longrightarrow e_{\mathrm{T}_{\xi}} \otimes e_{r-1, r, r} \otimes e_{\mathrm{T}_{\lambda \cup \alpha \downarrow>a}} \in \mathscr{H}_{n}^{(\succcurlyeq \lambda) \cup \beta}
$$

as required. (Note that $\gamma, \beta \notin \pi$ by Lemma 2.8.) See Fig. 8 for an example. 

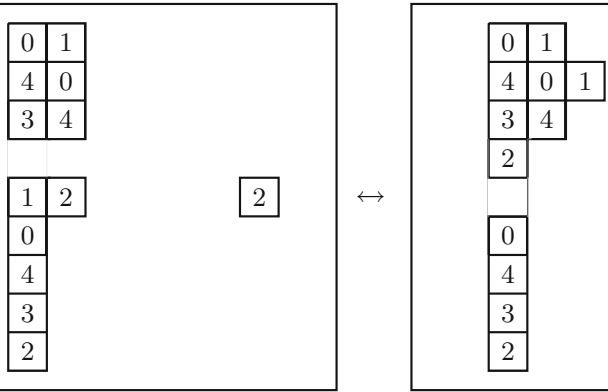

Fig. 8 Rewriting the first term after the equality in Eq. (2.18). We have moved the 1-box using case (iii) and this leaves us free to move the 2-boxes up their corresponding diagonals. The righthand-side is a box configuration which is strictly higher than $\lambda \cup \beta$ in the $\succ$-ordering
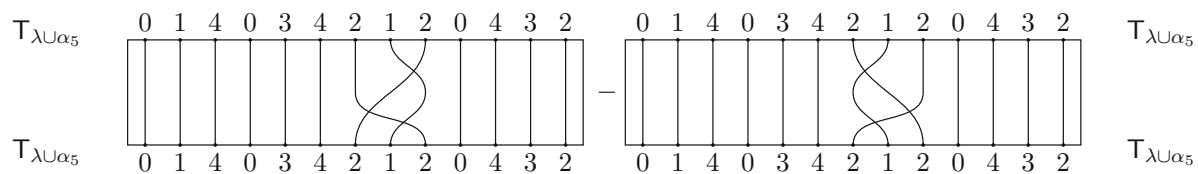

Fig. 9 The righthand-side of Eq. (2.18) for $\lambda=\left(2^{6}, 1^{3}\right)$ and $\alpha_{5}$ as in Fig. 4. The left diagram factors through the element $y_{\mathrm{T}_{\lambda \cup \alpha_{6}}^{\succ}}^{\succ}$; we will further manipulate this in Fig. 10 below

Fig. 10 The leftmost term in Fig.

9 rewritten in the form in Eq.

(2.20). This diagram factors

through $y_{\mathrm{T}_{\lambda \cup \alpha_{7}}}^{\succ}$. In this case

$\alpha=\alpha_{5}$ and $\beta=\alpha_{7}$ as in the first

case of Eq. (2.5)

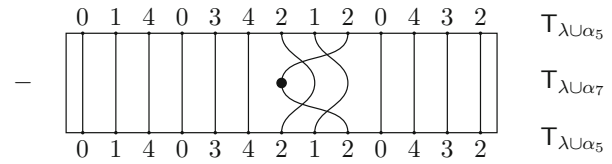

Proof of Eq. (2.7) for a given $\lambda$ and $\alpha$. We assume that Eq. (2.7) holds for all $\lambda \in \mathscr{P}_{h}(n-1)$. We set $v=\lambda \cup \alpha \in \mathscr{P}_{h}(n)$ and we assume that $\alpha$ is of residue $r \in \mathbb{Z} / e \mathbb{Z}$. We have that $e_{T_{\lambda \cup \alpha}}=y_{T_{\lambda \cup \alpha}}^{\succ}$. We have that

$$
y_{a} y_{\mathrm{T}_{\lambda \cup \alpha}}= \begin{cases}y_{a-1} y_{\mathrm{T}_{\lambda \cup \alpha}}+\psi_{b}^{a} y_{\mathrm{T}_{\lambda \cup \beta}} \psi_{a}^{b} & \text { if } r-1 \in \operatorname{res}\left(\operatorname{Adj}_{-G_{\succ}}(\alpha)\right) ; \\ y_{\mathrm{T}_{\lambda \cup \beta}} & \text { if } r-1 \notin \operatorname{res}\left(\operatorname{Adj}_{-G a r_{\succ}}(\alpha)\right) ;\end{cases}
$$

In the first case, this follows from case 3 of relation $\mathrm{R} 4$ and the commutativity relations, to see this note that the $(a-1)$ th strand has residue $r-1 \in \mathbb{Z} / e \mathbb{Z}$. In the second case, the statement follows from Lemma 2.8 and the fact that $b=a$. Letting $\beta^{\prime}$ be as in Sect. 2.4, we note that $y_{a-1} y_{\mathrm{T}_{\lambda}} \in \mathscr{H}_{n-1}^{\Downarrow \geq \lambda \cup \beta^{\prime}-\alpha^{\prime}}$ and so the first case of Eq. (2.21) is of the required form by our assumption for $\lambda \cup \beta^{\prime}-\alpha^{\prime}=\mu \succcurlyeq \lambda$.

Proof of Eq. (2.8). Let $j=\left(j_{1}, \ldots, j_{n-1}, r\right) \in(\mathbb{Z} / e \mathbb{Z})^{n}$. We can assume that $\operatorname{Shape}\left(\mathrm{J}_{\leqslant n-1}\right)=\lambda \in \mathscr{P}_{h}(n-1)$ as otherwise $e_{j} \otimes e_{j_{n}}=0 \otimes e_{r}=0$ by induction. Thus it remains to show that

$$
\tilde{\psi}_{\mathrm{T}_{\lambda}}^{J_{<n}} \tilde{\psi}_{\mathrm{J}_{<n}}^{\top_{\lambda}} \otimes e_{r} \in \pm \tilde{\psi}_{\mathrm{T}_{\nu}}^{J} \tilde{\psi}_{\jmath}^{\top_{\nu}}+\mathscr{H}_{n}^{\gg v} .
$$

We can associate this rightmost $r$-strand to the (unique) left-justified $r$-box $\alpha$ such that $\square \succ \alpha$ for all $\square \in \lambda$. (For example, $\square=[9,6,0]$ for $\lambda=\left(2^{3}, 1^{6}\right)$, see Fig. 4.) Thus every strand in the diagram is labelled by a box. We pull the strand labelled by $\alpha$ through the centre of the diagram (which is equal to the idempotent $e_{\mathrm{T}_{v}}$ ) one row at a time using Eq. (2.6). We can 
utilise Eq. (2.6) precisely when $\lambda \cup \alpha \notin \mathscr{P}_{h}(n)$. Therefore this process terminates when we reach the smallest addable $r$-box of $\lambda$ under the $\succ$-ordering, namely $\mathrm{J}^{-1}(n)$. Thus Eq. (2.8) follows.

Proposition 2.22 [4, Lemma 2.4 and Proposition 2.5] We let $\underline{w}, \underline{w}^{\prime}$ be any two choices of reduced expression for $w \in \mathfrak{S}_{n}$ and let $\underline{v}$ be any non-reduced expression for $w$. We have that

$$
\begin{aligned}
e_{\underline{i}} \psi_{\underline{w}} e_{\underline{j}} & =e_{\underline{i}} \psi_{\underline{w^{\prime}}} e_{\underline{j}}+\sum_{\underline{x}<\underline{w}, \underline{w^{\prime}}} e_{\underline{i}} \psi_{\underline{x}} f_{\underline{x}}(y) e_{\underline{j}} \\
e_{\underline{i}} \psi_{\underline{v}} e_{j} & =\sum_{\underline{x}<\underline{v}} e_{\underline{i}} \psi_{\underline{x}} e_{j} g_{\underline{x}}(y) \\
y_{k} e_{\underline{i}} \psi_{\underline{w}} e_{\underline{j}} & =e_{\underline{j}} \psi_{\underline{w}} e_{\underline{i}} y_{w(k)}+\sum_{\underline{x}<\underline{w}} e_{\underline{i}} \psi_{\underline{x}} e_{\underline{j}}
\end{aligned}
$$

for some $f_{\underline{x}}(y), g_{\underline{x}}(y) \in \mathcal{Y}_{n}$.

Proposition 2.23 Let $\mathbb{k}$ be an integral domain. The $\mathbb{k}$-algebra $\mathscr{H}_{n}^{\sigma}$ has spanning set

$$
\left\{\psi_{\mathrm{T}_{\lambda}}^{\mathrm{S}} \psi_{\mathrm{T}}^{\mathrm{T}_{\lambda}} \mid \mathrm{S}, \mathrm{T} \in \operatorname{Std}(\lambda), \lambda \in \mathscr{P}_{\underline{h}}(n)\right\} \text {. }
$$

Proof Let $d \in e_{\underline{i}} \mathscr{H}_{n}^{\sigma} e_{j}$ for some $l, j \in(\mathbb{Z} / e \mathbb{Z})^{n}$. By Eq. (2.8), we can rewrite $e_{j}$ (or equivalently $e_{\underline{i}}$ ) so that $d=\sum_{x, y \in \mathfrak{S}_{n}} e_{\underline{i}} a_{x} e_{\mathrm{T}_{\lambda}} a_{y} e_{\underline{j}}$ for some $a_{x}, a_{y}$ which are linear combinations of KLR elements tracing out some bijections $\bar{x}, y \in \mathfrak{S}_{n}$ respectively (but are possibly decorated with dots and need not be reduced). It remains to show that $a_{x}, a_{y} \in \mathscr{H}_{n}^{\sigma}$ can be assumed to be reduced and undecorated. We establish this by induction along the Bruhat order, by working modulo the span of elements

$$
\operatorname{Span}_{\mathbb{k}}\left\{\psi_{\underline{u}} e_{\mathrm{T}_{\lambda}} \psi_{\underline{v}} \mid u<x \text { or } v<y\right\}+\mathscr{H}_{n}^{i>\lambda} .
$$

If $\underline{x}$ is not reduced $\psi_{\underline{x}} e_{\mathrm{T}_{\lambda}} a_{y}$ is zero modulo (2.26) by Eq. (2.23). Given two choices $\underline{x}, \underline{x}^{\prime}$ of reduced expression for $x \in \mathfrak{S}_{n}$ we have that $\left(\psi_{\underline{x}}-\psi_{\underline{x}^{\prime}}\right) e_{\mathrm{T}_{\lambda}} a_{y}$ belongs to (2.26) by Eq. (2.22) followed by Eq. (2.7). Finally, if $a_{x}$ is obtained from $\psi_{\underline{x}}$ by adding a linear combination of dot decorations (at any points within the expression $\psi_{\underline{x}}=\psi_{s_{i_{1}}} \ldots \psi_{s_{i_{k}}}$ ) then $\psi_{\underline{x}} e_{T_{\lambda}} a_{y}$ is zero modulo (2.26) by Eq. (2.24) followed by Eq. (2.7). Thus $\mathscr{H}_{n}^{\Downarrow \lambda} / \mathscr{H}_{n}^{\gg \lambda}$ is spanned by elements of the form

$\left\{\psi_{\underline{x}} e_{\top_{\lambda}} \psi_{\underline{y}} \mid\right.$ for $\underline{x}, \underline{y}$ arbitrary choices of fixed reduced expressions of $\left.x, y \in \mathfrak{S}_{n}\right\}+\mathscr{H}_{n}^{\gg \lambda}$.

It remains to show that a spanning set is given by the elements $x=w_{\mathrm{T}_{\lambda}}^{\mathrm{S}}, y=w_{\mathrm{T}}^{\mathrm{T}_{\lambda}}$ for $S, T \in \operatorname{Std}(\lambda)$.

Given $\mathrm{T} \in \operatorname{CStd}(\lambda) \backslash \operatorname{Std}(\lambda)$, we have that $w_{\top}^{\top_{\lambda}}$ has a pair of crossing strands from $1 \leqslant i<$ $j \leqslant n$ to $1 \leqslant w_{\mathrm{T}}^{\mathrm{T}_{\lambda}}(j)<w_{\mathrm{T}}^{\mathrm{\top}_{\lambda}}(i) \leqslant n$ such that $\mathrm{T}_{\lambda}^{-1}(i)=[r, c, m]$ and $\mathrm{T}_{\lambda}^{-1}(j)=[r, c+1, m]$ are in the same row and in particular so that $i=j-1$. It suffices to show that $\psi_{\underline{x}} e_{\mathrm{T}_{\lambda}} \psi_{\underline{y}}$ belongs to the ideal $\mathscr{H}_{n}^{i \lambda \lambda}$ for a preferred choice of $y$; we choose $y=s_{i} \underline{w}$ (for some $w \in \mathfrak{S}_{n}$ such that $s_{i} w=y$ ). Thus it remains to show that $e_{\mathrm{T}_{\lambda}} \psi_{s_{i} \underline{w}} \in \overline{\mathscr{H}}_{n}^{i>\lambda}$. However, this immediately follows from Eq. (2.6) because $e_{\mathrm{T}_{\lambda}} \psi_{s_{i}}=\psi_{s_{i}} e_{s_{i}\left(\mathrm{~T}_{\lambda}\right)}$ and we have that $e_{s_{i}\left(\mathrm{~T}_{\lambda}\right)} \in \mathscr{H}_{n}^{\succeq \alpha}$ for $\alpha=Y_{\mathrm{T}_{\lambda}^{-1}(i+1)}(\lambda) \succcurlyeq \lambda$.

Given any $\mathrm{T} \in \operatorname{RStd}(\lambda) \backslash \operatorname{Std}(\lambda)$ we let $k$ be minimal such that $\mathrm{T} \downarrow<k \in \operatorname{Std}(\mu)$ for some $\mu \in \mathscr{P}_{\underline{h}}(k-1)$ and Shape $(\mathrm{T} \downarrow \leqslant k)=v$ for some $v \notin \mathscr{P}_{\underline{h}}(k)$. We have that $e_{\mathrm{T}}=e_{\mathrm{T}_{\leqslant k}} \nabla e_{\mathrm{T}_{>k}}$ where $\operatorname{Shape}\left(e_{\mathrm{T}_{\leqslant k}}\right)=v \in \mathscr{C}_{\underline{h}}(k) \backslash \mathscr{P}_{\underline{h}}(k)$ and so $e_{\mathrm{T}_{\leqslant k}} \in \mathscr{H}_{k}^{\gg v}$ by Eq. (2.6) and so $e_{\mathrm{T}} \in \mathscr{H}_{n} \gg \lambda$ by concatenation and the definition of $\succcurlyeq$. This implies that $e_{\mathrm{T}_{\lambda}} \psi_{\mathrm{T}}^{\mathrm{T}_{\lambda}}=\psi_{\mathrm{T}}^{\mathrm{T}_{\lambda}} e_{\mathrm{T}} \in \mathscr{H}_{n}^{\gg \lambda}$, as required. 
Theorem 2.24 Let $\mathbb{k}$ be an integral domain. The $\mathbb{k}$-algebra $\mathscr{H}_{n}^{\sigma}$ is graded cellular with basis

$$
\left\{\psi_{\mathrm{T}_{\lambda}}^{\mathrm{S}} \psi_{\mathrm{T}}^{\mathrm{T}_{\lambda}} \mid \mathrm{S}, \mathrm{T} \in \operatorname{Std}(\lambda), \lambda \in \mathscr{P}_{\underline{h}}(n)\right\}
$$

anti-involution $*$ and the degree function $\operatorname{deg}: \operatorname{Std} \rightarrow \mathbb{Z}$. For $\mathbb{k}$ a field, $\mathscr{H}_{n}^{\sigma}$ is quasihereditary.

Proof We first prove that the spanning set of Proposition 2.23 is a $\mathbb{k}$-basis. We will show that the rank, as a $\mathbb{k}$-module, of $\mathcal{H}_{n}^{\sigma} \mathrm{y}_{\underline{h}} \mathcal{H}_{n}^{\sigma}$ is less than or equal to $\sum_{\lambda \in \mathscr{P}(n) \backslash \mathscr{P}_{\underline{h}}(n)}|\operatorname{Std}(\lambda)|^{2}$. The ( $\succ$ )-ordering does not give us an easily constructible basis of $\mathcal{H}_{n}^{\sigma}$ (see [5]). Hu and Mathas [7, Main Theorem] have shown that the classical ordering $\triangleright$ does give us an easily constructible basis $\left\{\psi_{\mathrm{S}_{\lambda}}^{\mathrm{S}} y_{\mathrm{S}_{\lambda}}^{\triangleright} \psi_{\mathrm{T}}^{\mathrm{S}_{\lambda}} \mid \mathrm{S}, \mathrm{T} \in \mathrm{Std}(\lambda)\right\}$ and presentations of all these cell-modules are given in [9, Definition 5.9]. We claim that $y_{h}$ annihilates any ( $\triangleright$ )-cell-module labelled by a $\lambda \in \mathscr{P}_{\underline{h}}(n)$; in other words $\mathrm{y}_{\underline{h}} \psi_{\mathrm{S}_{\lambda}}^{\mathrm{S}} y_{\mathrm{S}_{\lambda}}^{\triangleright} \in \mathcal{H}_{n}^{\triangleright \bar{\lambda}}$ for $\lambda \in \mathscr{P}_{\underline{h}}(n)$. Once we have proven the claim, we will deduce that the ideal is of the required rank, thus the spanning set is linearly independent (and hence a basis) as required. The algebra is then cellular (by its construction via idempotent ideals) with the stated basis (since we have a spanning set of the required rank). Finally, we note that each layer of the cell chain contains an idempotent $e_{T_{\lambda}}$ and so the algebra is quasi-hereditary, as required. We now turn to the proof of the claim. If $m<\ell-1$ and $h_{m}<\sigma_{m+1}-\sigma_{m}$, or if $m=\ell-1$ and $h_{\ell-1}<e+\sigma_{0}-\sigma_{\ell-1}$ then the $m$ th summand

$$
y_{\left(\emptyset, \cdots, \emptyset,\left(h_{m}+1\right), \emptyset, \cdots, \emptyset\right)}^{\succ} \otimes 1_{\mathcal{H}_{n-h_{m}}^{\sigma}}=e_{\left(\sigma_{m}, \sigma_{m}+1, \ldots, \sigma_{m}+h_{m}\right)} \otimes 1_{\mathcal{H}_{n-h_{m}-1}^{\sigma}}
$$

in Eq. (2.4) is an idempotent whose residue sequence is not equal to that of any tableau $\mathrm{S} \in \operatorname{Std}(\lambda)$ for $\lambda \in \mathscr{P}_{h}(n)$ and therefore the claim is immediate. If $h_{m}=\sigma_{m+1}-\sigma_{m}$, then the term in (2.29) is nilpotent and equal to

$$
y_{h_{m}+1} e \boldsymbol{\top}_{\left(\emptyset, \ldots, \emptyset,\left(h_{m}+1\right), \emptyset, \ldots, \emptyset\right)} \otimes 1_{\mathcal{H}_{n-h_{m}-1}^{\sigma}}=y_{h_{m}+1} e^{\top_{\left(\emptyset, \ldots, \emptyset,\left(h_{m}\right),(1), \emptyset, \ldots, \emptyset\right)}} \otimes 1_{\mathcal{H}_{n-h_{m}-1}^{\sigma}} .
$$

The idempotent in (2.30) annihilates $\psi_{\mathrm{S}_{\lambda}}^{\mathrm{S}} y_{\lambda}^{\triangleright}$ unless $\mathrm{S} \downarrow_{\left\{1, \ldots, h_{m}+1\right\}}$ is equal to $\mathrm{T}_{\left(\emptyset, \ldots, \emptyset,\left(h_{m}\right),(1), \emptyset, \ldots, \emptyset\right)}$. We now suppose that $\mathrm{S} \downarrow_{\left\{1, \ldots, h_{m}+1\right\}}$ is of this form and we set $s_{m+1} \equiv$ $\sigma_{m+1} \bmod e$.

Since $\lambda \in \mathscr{P}_{h}(n)$, we observe that $[1,1, m+1]$ is the unique box in $\lambda$ of residue $s_{m+1} \in$ $\mathbb{Z} / e \mathbb{Z}$ in which we can place the integer $h_{m}+1$ (or any integer smaller than $h_{m}+1$ ) without violating the standard condition, by Lemma 2.8 . The presentation of the Specht module in [9, Definition 5.9] implies that (i) $\psi_{\underline{w}} y_{\lambda}^{\triangleright} \in \mathcal{H}_{n}^{\triangleright \lambda}$ for any $w \neq w_{\mathrm{S}_{\lambda}}^{\mathrm{S}}$ for some $\mathrm{S} \in \operatorname{Std}(\lambda)$ with $\lambda \in \mathscr{P}_{\underline{h}}(n)$ (since every $(\triangleright)$ )-Garnir belt has fewer than $e$ boxes) and (ii) $y_{k} y_{\lambda}^{\triangleright} \in \mathcal{H}_{n}^{\triangleright \lambda}$ for any $1 \leqslant k \leqslant n$.

We are now ready to prove the claim. Using Eq. (2.24), we move the dot at the top of $y_{h_{m}+1} \psi_{S_{\lambda}}^{\mathrm{S}} y_{\lambda}^{\triangleright}$ down the $\left(h_{m}+1\right)$ th strand to obtain a linear combination of undecorated diagrams (in which we have undone some number of crossings $s_{m}$-strands) and $\psi_{\mathrm{S}_{\lambda}}^{\mathrm{S}} y_{\mathrm{S}_{\lambda}(1,1, m+1)} y_{\lambda}^{\triangleright}$. By our above observation, all of these undecorated diagrams are labelled by non-standard $\lambda$-tableaux. Therefore all of these terms (and hence $y_{h_{m}+1} \psi_{\mathrm{S}_{\lambda}}^{\mathrm{S}} y_{\lambda}^{\triangleright}$ ) are zero, by (i) and (ii). The claim and result follow.

Let $\mathbb{k}$ be an integral domain. We define the standard or Specht modules of $\mathscr{H}_{n}^{\sigma}$ as follows,

$$
\mathbf{S}_{\mathbb{k}}(\lambda)=\left\{\psi_{\mathrm{T}_{\lambda}}^{\mathrm{S}}+\mathscr{H}^{>\lambda} \mid \mathrm{S} \in \operatorname{Std}(\lambda)\right\}
$$

for $\lambda \in \mathscr{P}_{\underline{h}}(n)$. We immediately deduce the following corollary of Theorem 2.24.

Corollary 2.25 The module $\mathbf{S}_{\mathbb{k}}(\lambda)$ is the module generated by $e_{\mathrm{T}_{\lambda}}$ subject to the following relations: 
- $e_{\underline{i}} e_{\mathrm{T}_{\lambda}}=\delta_{\underline{i}, \operatorname{res}\left(\mathrm{T}_{\lambda}\right)} e_{\mathrm{T}_{\lambda}}$ for $\underline{i} \in(\mathbb{Z} / e \mathbb{Z})^{n}$;

- $y_{k} e_{\mathrm{T}_{\lambda}}=0$ for $1 \leqslant k \leqslant n$;

- $\psi_{k} e_{\mathrm{T}_{\lambda}}=0$ for any $1 \leqslant k<n$ such that $s_{k}\left(\mathrm{~T}_{\lambda}\right)$ is not row standard;

- $\psi_{T_{\lambda}}^{S} e_{T_{\lambda}}=0$ for $S \in \operatorname{RStd}(\lambda) \backslash \operatorname{Std}(\lambda)$.

Proof We have already checked that all of these relations hold (and so one can define a homomorphism from the abstractly defined module with this presentation to $\mathbf{S}_{\mathbb{k}}(\lambda)$ ) it only remains to check that these relations will suffice (i.e. the homomorphism is surjective). We know that $\mathbf{S}_{\mathbb{k}}(\lambda)$ has a basis indexed by standard tableaux and so the result follows.

We now recall that the cellular structure allows us to define bilinear forms, for each $\lambda \in \mathscr{P}_{\underline{h}}(n)$, there is a bilinear form $\langle,\rangle^{\lambda}$ on $\mathbf{S}_{\mathbb{k}}(\lambda)$, which is determined by

$$
\psi_{\mathrm{S}}^{\mathrm{T}_{\lambda}} \psi_{\mathrm{T}_{\lambda}}^{\top} \equiv\left\langle\psi_{\mathrm{T}_{\lambda}}^{\mathrm{S}}, \psi_{\mathrm{T}_{\lambda}}^{\top}\right\rangle^{\lambda} e_{\mathrm{T}_{\lambda}} \quad\left(\bmod \mathcal{H}^{\succ \lambda}\right)
$$

for any $S, T \in \operatorname{Std}(\lambda)$. Let $\mathbb{k}$ be a field of arbitrary characteristic. Factoring out by the radicals of these forms, we obtain a complete set of non-isomorphic simple $\mathscr{H}_{n}^{\sigma}$-modules

$$
\mathbf{D}_{\mathbb{k}}(\lambda)=\mathbf{S}_{\mathbb{k}}(\lambda) / \operatorname{rad}\left(\mathbf{S}_{\mathbb{k}}(\lambda)\right), \lambda \in \mathscr{P}_{\underline{h}}(n) .
$$

Proposition 2.26 Let $\lambda \in \mathscr{P}_{\underline{h}}(n)$ and let $A_{1} \succ A_{2} \succ \cdots \succ A_{z}$ denote the removable boxes of $\lambda$, totally ordered according to the $\succeq$-ordering. The restriction of $\mathbf{S}_{\mathbb{k}}(\lambda)$ has an $\mathscr{H}_{n-1}^{\sigma}$-module filtration

$$
0=\mathbf{S}^{z+1, \lambda} \subset \mathbf{S}^{z, \lambda} \subset \cdots \subset \mathbf{S}^{1, \lambda}=\operatorname{Res}_{\mathscr{H}_{n-1}^{\sigma}}\left(\mathbf{S}_{\mathbb{k}}(\lambda)\right)
$$

given by

$$
\mathbf{S}^{x, \lambda}=\mathbb{k}\left\{\psi_{T_{\lambda}}^{S} \mid \text { Shape }\left(\mathrm{S}_{\leqslant n-1}\right)=\lambda-A_{y} \text { for some } z \geqslant y \geqslant x\right\}
$$

For each $1 \leqslant r \leqslant z$, we have that

$$
\varphi_{r}: \mathbf{S}\left(\lambda-A_{r}\right)\left\langle\operatorname{deg}\left(A_{r}\right)\right\rangle \cong \mathbf{S}^{r, \lambda} / \mathbf{S}^{r+1, \lambda} \quad: \psi_{\mathrm{S}_{\leqslant n-1}} \mapsto \psi_{\mathrm{S}_{\leqslant n-1}} \circ \psi_{\mathrm{T}_{\lambda}\left(A_{r}\right)}^{n}
$$

Proof On the level of $\mathbb{k}$-modules, this is clear. Lifting this to $\mathscr{H}_{n-1}^{\sigma}$-modules is a standard argument which proceeds by checking the relations of Corollary 2.25 in a routine manner.

\section{General light leaves bases for quiver Hecke algebras}

The principal idea of categorical Lie theory is to replace existing structures (combinatorics, bases, and presentations of Hecke algebras) with richer structures which keep track of more information. In this section, we replace the classical tableaux combinatorics of symmetric groups (and quiver Hecke algebras) with that of paths in an alcove geometry. This will allow us to construct "light leaves" bases of these algebras, for which $p$-Kazhdan-Lusztig is bakedin to the very definition. The light leaves bases of $\mathbf{S}_{\mathbb{k}}(\lambda)$ are constructed in such a way as to keep track of not just the point $\lambda \in \mathbb{E}_{h}$ (or rather the single path, $T_{\lambda}$, to the point $\lambda$ ) but of the many different ways we can get to the point $\lambda$ by a reduced path/word in the alcove geometry. This extra generality is essential when we wish to write bases in terms of "2-generators" of the algebras of interest. 


\subsection{The alcove geometry}

For ease of notation, we set $H_{m}=h_{0}+\cdots+h_{m}$ for $0 \leqslant m<\ell$, and $h=h_{0}+\cdots+h_{\ell-1}$. For each $1 \leqslant i \leqslant n$ and $0 \leqslant m<\ell$ we let $\varepsilon_{i, m}:=\varepsilon_{\left(h_{0}+\cdots+h_{m-1}\right)+i}$ denote a formal symbol, and define an $h$-dimensional real vector space

$$
\mathbb{E}_{\underline{h}}=\bigoplus_{\substack{0 \leqslant m<\ell \\ 1 \leqslant i \leqslant h_{m}}} \mathbb{R} \varepsilon_{i, m}
$$

and $\overline{\mathbb{E}}_{h}$ to be the quotient of this space by the one-dimensional subspace spanned by

$$
\sum_{\substack{0 \leqslant m<\ell \\ 1 \leqslant i \leqslant h_{m}}} \varepsilon_{i, m} .
$$

We have an inner product $\langle$,$\rangle on \mathbb{E}_{\underline{h}}$ given by extending linearly the relations

$$
\left\langle\varepsilon_{i, p}, \varepsilon_{j, q}\right\rangle=\delta_{i, j} \delta_{p, q}
$$

for all $1 \leqslant i, j \leqslant n$ and $0 \leqslant p, q<\ell$, where $\delta_{i, j}$ is the Kronecker delta. We identify $\lambda \in \mathcal{C}_{\underline{h}}(n)$ with an element of the integer lattice inside $\mathbb{E}_{\underline{h}}$ via the map

$$
\lambda \longmapsto \sum_{\substack{0 \leqslant m<\ell \\ 1 \leqslant i \leqslant h_{m}}}\left(\lambda^{(m)}\right)_{i}^{T} \varepsilon_{i, m}
$$

where $(-)^{T}$ is the transpose map. We let $\Phi$ denote the root system of type $A_{h-1}$ consisting of the roots

$$
\left\{\varepsilon_{i, p}-\varepsilon_{j, q}: 0 \leqslant p, q<\ell, 1 \leqslant i \leqslant h_{p}, 1 \leqslant j \leqslant h_{q}, \text { with }(i, p) \neq(j, q)\right\}
$$

and $\Phi_{0}$ denote the root system of type $A_{h_{0}-1} \times \cdots \times A_{h_{\ell-1}-1}$ consisting of the roots

$$
\left\{\varepsilon_{i, m}-\varepsilon_{j, m}: 0 \leqslant m<\ell, 1 \leqslant i \neq j \leqslant h_{m}\right\} .
$$

We choose $\Delta$ (respectively $\Delta_{0}$ ) to be the set of simple roots inside $\Phi$ (respectively $\Phi_{0}$ ) of the form $\varepsilon_{t}-\varepsilon_{t+1}$ for some $t$. Given $r \in \mathbb{Z}$ and $\alpha \in \Phi$ we define $s_{\alpha, r e}$ to be the reflection which acts on $\mathbb{E}_{\underline{h}}$ by

$$
s_{\alpha, r e} x=x-(\langle x, \alpha\rangle-r e) \alpha
$$

The group generated by the $s_{\alpha, 0}$ with $\alpha \in \Phi$ (respectively $\alpha \in \Phi_{0}$ ) is isomorphic to the symmetric group $\mathfrak{S}_{h}$ (respectively to $\mathfrak{S}_{f}:=\mathfrak{S}_{h_{0}} \times \cdots \times \mathfrak{S}_{h_{\ell-1}}$ ), while the group generated by the $s_{\alpha, r e}$ with $\alpha \in \Phi$ and $r \in \mathbb{Z}$ is isomorphic to $\widehat{\mathfrak{S}}_{h}$, the affine Weyl group of type $A_{h-1}$. We set $\alpha_{0}=\varepsilon_{h}-\varepsilon_{1}$ and $\Pi=\Delta \cup\left\{\alpha_{0}\right\}$. The elements $S=\left\{s_{\alpha, 0}: \alpha \in \Delta\right\} \cup\left\{s_{\alpha_{0},-e}\right\}$ generate $\widehat{\mathfrak{S}}_{h}$.

Notation 3.1 We shall frequently find it convenient to refer to the generators in $S$ in terms of the elements of $\Pi$, and will abuse notation in two different ways. First, we will write $s_{\alpha}$ for $s_{\alpha, 0}$ when $\alpha \in \Delta$ and $s_{\alpha_{0}}$ for $s_{\alpha_{0},-e}$. This is unambiguous except in the case of the affine reflection $s_{\alpha_{0},-e}$, where this notation has previously been used for the element $s_{\alpha, 0}$. As the element $s_{\alpha_{0}, 0}$ will not be referred to hereafter this should not cause confusion. Second, we will write $\alpha=\varepsilon_{i}-\varepsilon_{i+1}$ in all cases; if $i=h$ then all occurrences of $i+1$ should be interpreted modulo $h$ to refer to the index 1 . 
We shall consider a shifted action of the affine Weyl group $\widehat{\mathfrak{S}}_{h}$ on $\mathbb{E}_{h, l}$ by the element $\rho:=\left(\rho_{0}, \rho_{2}, \ldots, \rho_{\ell-1}\right) \in \mathbb{Z}^{h}$ where $\rho_{m}:=\left(\sigma_{m}+h_{m}-1, \sigma_{m}+h_{m}-2, \ldots, \sigma_{m}\right) \in \mathbb{Z}^{h_{m}}$, that is, given an element $w \in \widehat{\mathfrak{S}}_{h}$, we set $w \cdot x=w(x+\rho)-\rho$. This shifted action induces a well-defined action on $\overline{\mathbb{E}}_{\underline{h}}$; we will define various geometric objects in $\mathbb{E}_{\underline{h}}$ in terms of this action, and denote the corresponding objects in the quotient with a bar without further comment. We let $\mathbb{E}(\alpha, r e)$ denote the affine hyperplane consisting of the points

$$
\mathbb{E}(\alpha, r e)=\left\{x \in \mathbb{E}_{\underline{h}} \mid s_{\alpha, r e} \cdot x=x\right\} .
$$

Note that our assumption that $e>h_{0}+\cdots+h_{\ell-1}$ implies that the origin does not lie on any hyperplane. Given a hyperplane $\mathbb{E}(\alpha, r e)$ we remove the hyperplane from $\mathbb{E}_{h}$ to obtain two distinct subsets $\mathbb{E}^{>}(\alpha, r e)$ and $\mathbb{E}^{<}(\alpha, r e)$ where the origin lies in $\mathbb{E}^{<}(\alpha, r e)$. The connected components of

$$
\overline{\mathbb{E}}_{\underline{h}} \backslash\left(\cup_{\alpha \in \Phi_{0}} \overline{\mathbb{E}}(\alpha, 0)\right)
$$

are called chambers. The dominant chamber, denoted $\overline{\mathbb{E}}_{\underline{h}}^{+}$, is defined to be

$$
\overline{\mathbb{E}}_{\underline{h}}^{+}=\bigcap_{\alpha \in \Phi_{0}} \overline{\mathbb{E}}^{<}(\alpha, 0) .
$$

The connected components of

$$
\overline{\mathbb{E}}_{\underline{h}} \backslash\left(\cup_{\alpha \in \Phi, r \in \mathbb{Z}} \overline{\mathbb{E}}(\alpha, r e)\right)
$$

are called alcoves, and any such alcove is a fundamental domain for the action of the group $\widehat{\mathfrak{S}}_{h}$ on the set Alc of all such alcoves. We define the fundamental alcove $A_{0}$ to be the alcove containing the origin (which is inside the dominant chamber). We have a bijection from $\widehat{\mathfrak{S}}_{h}$ to Alc given by $w \longmapsto w A_{0}$. Under this identification Alc inherits a right action from the right action of $\widehat{\mathfrak{S}}_{h}$ on itself. Consider the subgroup

$$
\mathfrak{S}_{f}:=\mathfrak{S}_{h_{0}} \times \cdots \times \mathfrak{S}_{h_{\ell-1}} \leqslant \widehat{\mathfrak{S}}_{h} .
$$

The dominant chamber is a fundamental domain for the action of $\mathfrak{S}_{f}$ on the set of chambers in $\overline{\mathbb{E}}_{h}$. We let $\mathfrak{S}^{f}$ denote the set of minimal length representatives for right cosets $\mathfrak{S}_{f} \backslash \widehat{\mathfrak{S}}_{h}$. So multiplication gives a bijection $\mathfrak{S}_{f} \times \mathfrak{S}^{f} \rightarrow \widehat{\mathfrak{S}}_{h}$. This induces a bijection between right cosets and the alcoves in our dominant chamber. Under this identification, alcoves are partially ordered by the Bruhat-ordering on $\widehat{\mathfrak{S}}_{h}$ which is a coarsening of the opposite of the order $\gg$.

If the intersection of a hyperplane $\overline{\mathbb{E}}(\alpha, r e)$ with the closure of an alcove $A$ is generically of codimension one in $\overline{\mathbb{E}}_{h}$ then we call this intersection a wall of $A$. The fundamental alcove $A_{0}$ has walls corresponding to $\overline{\mathbb{E}}(\alpha, 0)$ with $\alpha \in \Delta$ together with an affine wall $\overline{\mathbb{E}}\left(\alpha_{0},-e\right)$. We will usually just write $\overline{\mathbb{E}}(\alpha)$ for the walls $\overline{\mathbb{E}}(\alpha, 0)$ (when $\alpha \in \Delta$ ) and $\overline{\mathbb{E}}(\alpha,-e)$ (when $\alpha=\alpha_{0}$ ). We regard each of these walls as being labelled by a distinct colour (and assign the same colour to the corresponding element of $S$ ). Under the action of $\widehat{\mathfrak{S}}_{h}$ each wall of a given alcove $A$ is in the orbit of a unique wall of $A_{0}$, and thus inherits a colour from that wall. We will sometimes use the right action of $\widehat{\mathfrak{S}}_{h}$ on Alc. Given an alcove $A$ and an element $s \in S$, the alcove $A s$ is obtained by reflecting $A$ in the wall of $A$ with colour corresponding to the colour of $s$. With this observation it is now easy to see that if $w=s_{1} \ldots s_{t}$ where the $s_{i}$ are in $S$ then $w A_{0}$ is the alcove obtained from $A_{0}$ by successively reflecting through the walls corresponding to $s_{1}$ up to $s_{h}$. We will call a multipartition regular if its image in $\overline{\mathbb{E}}_{h, l}$ lies 
in some alcove; those multipartitions whose images lies on one or more walls will be called singular.

\subsection{Paths in the geometry}

We now develop a path combinatorics inside our geometry. Given a map $p:\{1, \ldots, n\} \rightarrow$ $\{1, \ldots, h\}$ we define points $\mathrm{P}(k) \in \mathbb{E}_{\underline{h}}$ by

$$
\mathrm{P}(k)=\sum_{1 \leqslant i \leqslant k} \varepsilon_{p(i)}
$$

for $1 \leqslant i \leqslant n$. We define the associated path of length $n$ by

$$
\mathrm{P}=(\varnothing=\mathrm{P}(0), \mathrm{P}(1), \mathrm{P}(2), \ldots, \mathrm{P}(n))
$$

and we say that the path has shape $\pi=\mathrm{P}(n) \in \mathbb{E}_{\underline{h}}$. We also denote this path by

$$
\mathrm{P}=\left(\varepsilon_{p(1)}, \ldots, \varepsilon_{p(n)}\right) .
$$

Given $\lambda \in \mathscr{C}_{h}(n)$ we let Path $(\lambda)$ denote the set of paths of length $n$ with shape $\lambda$. We define $\operatorname{Path}_{h}(\lambda)$ to be the subset of Path $(\lambda)$ consisting of those paths lying entirely inside the dominant chamber; i.e. those $\mathrm{P}$ such that $\mathrm{P}(i)$ is dominant for all $0 \leqslant i \leqslant n$. We let $\operatorname{Path}_{\underline{h}}(n)=\cup_{\lambda \in \mathscr{P}_{h}(n)} \operatorname{Path}_{\underline{h}}(\lambda)$.

Given a path T defined by such a map $p$ of length $n$ and shape $\lambda$ we can write each $p(j)$ uniquely in the form $\varepsilon_{p(j)}=\varepsilon_{m_{j}, c_{j}}$ where $0 \leqslant m_{j}<\ell$ and $1 \leqslant c_{j} \leqslant h_{j}$. We record these elements in a tableau of shape $\lambda^{T}$ by induction on $j$, where we place the positive integer $j$ in the first empty box in the $c_{j}$ th column of component $m_{j}$. By definition, such a tableau will have entries increasing down columns; if $\lambda$ is a multipartition then the entries also increase along rows if and only if the given path is in $\operatorname{Path}_{h}(\lambda)$, and hence there is a bijection between $\operatorname{Path}_{h}(\lambda)$ and $\operatorname{Std}(\lambda)$. For this reason we will sometimes refer to paths as tableaux, to emphasise that what we are doing is generalising the classical tableaux combinatorics for the symmetric group.

Example 3.2 Let $\sigma=(0,3,6) \in \mathbb{Z}^{3}$ and $e=9$. For $\lambda=\left((2,1),(2,1),\left(1^{3}\right)\right)$, the standard $\lambda$-tableaux of Example 2.5 correspond to the paths

$$
\begin{aligned}
\mathrm{T}_{\lambda} & =\left(\varepsilon_{1}, \varepsilon_{2}, \varepsilon_{3}, \varepsilon_{4}, \varepsilon_{5}, \varepsilon_{1}, \varepsilon_{3}, \varepsilon_{4}, \varepsilon_{5}, \varepsilon_{1}, \varepsilon_{3}, \varepsilon_{5}\right) \\
\mathrm{S} & =\left(\varepsilon_{1}, \varepsilon_{1}, \varepsilon_{3}, \varepsilon_{5}, \varepsilon_{4}, \varepsilon_{2}, \varepsilon_{3}, \varepsilon_{4}, \varepsilon_{5}, \varepsilon_{1}, \varepsilon_{3}, \varepsilon_{5}\right)
\end{aligned}
$$

Given a path $\mathrm{P}$ we define

$$
\operatorname{res}(\mathrm{P})=(\operatorname{resp}(1), \ldots, \operatorname{resp}(n))
$$

where resp $(i)$ denotes the residue of the box labelled by $i$ in the tableau corresponding to $\mathrm{P}$.

Given paths $\mathrm{P}=\left(\varepsilon_{p(1)}, \ldots, \varepsilon_{p(n)}\right)$ and $\mathrm{Q}=\left(\varepsilon_{q(1)}, \ldots, \varepsilon_{q(n)}\right)$ we say that $\mathrm{P} \sim \mathrm{Q}$ if there exists an $\alpha \in \Phi$ and $r \in \mathbb{Z}$ and $s \leqslant n$ such that

$$
\mathrm{P}(s) \in \mathbb{E}(\alpha, r e) \quad \text { and } \quad \varepsilon_{q(t)}= \begin{cases}\varepsilon_{p(t)} & \text { for } 1 \leqslant t \leqslant s \\ s_{\alpha} \varepsilon_{p(t)} & \text { for } s+1 \leqslant t \leqslant n .\end{cases}
$$

In other words the paths $\mathrm{P}$ and $\mathrm{Q}$ agree up to some point $\mathrm{P}(s)=\mathrm{Q}(s)$ which lies on $\mathbb{E}(\alpha, r e)$, after which each $\mathrm{Q}(t)$ is obtained from $\mathrm{P}(t)$ by reflection in $\mathbb{E}(\alpha, r e)$. We extend $\sim$ by transitivity to give an equivalence relation on paths, and say that two paths in the same 

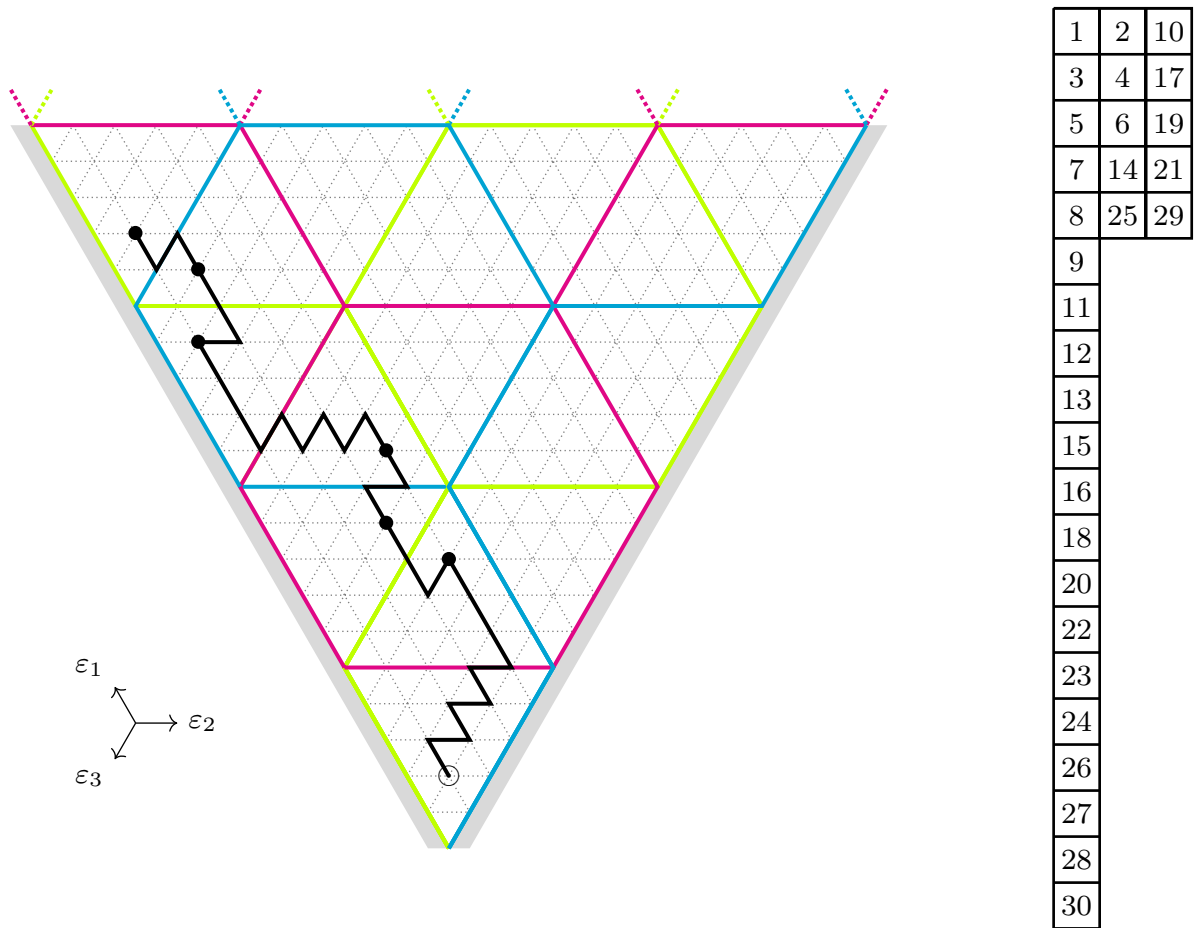

Fig. 11 An alcove path in $\overline{\mathbb{E}}_{3,1}^{+}$in $\operatorname{Path}_{h}\left(3^{5}, 1^{15}\right)$ and the corresponding tableau in $\operatorname{Std}\left(3^{5}, 1^{15}\right)$. The black vertices denote vertices on the path in the orbit of the origin

equivalence class are related by a series of wall reflections of paths and given $S \in \operatorname{Path}_{\underline{h}}(n)$ we set $[\mathrm{S}]=\left\{\mathrm{T} \in \operatorname{Path}_{\underline{h}}(n) \mid \mathrm{S} \sim \mathrm{T}\right\}$.

We recast the degree of a tableau in the path-theoretic setting as follows.

Definition 3.3 Given a path $\mathrm{S}=(\mathrm{S}(0), \mathrm{S}(1), \mathrm{S}(2), \ldots, \mathrm{S}(n))$ we set $\operatorname{deg}(\mathrm{S}(0))=0$ and define

$$
\operatorname{deg}(\mathrm{S})=\sum_{1 \leqslant k \leqslant n} d(\mathrm{~S}(k), \mathrm{S}(k-1)),
$$

where $d(\mathrm{~S}(k), \mathrm{S}(k-1))$ is defined as follows. For $\alpha \in \Phi$ we set $d_{\alpha}(\mathrm{S}(k), \mathrm{S}(k-1))$ to be

- +1 if $\mathrm{S}(k-1) \in \mathbb{E}(\alpha, r e)$ and $\mathrm{S}(k) \in \mathbb{E}^{<}(\alpha, r e)$;

- -1 if $\mathrm{S}(k-1) \in \mathbb{E}^{>}(\alpha, r e)$ and $\mathrm{S}(k) \in \mathbb{E}(\alpha, r e)$;

- 0 otherwise as illustrated in (Fig. 12).

We let

$$
\operatorname{deg}(\mathrm{S})=\sum_{1 \leqslant k \leqslant n} \sum_{\alpha \in \Phi} d_{\alpha}(\mathrm{S}(k-1), \mathrm{S}(k)) .
$$

We say that $\mathrm{P}=\left(\varepsilon_{p(1)}, \ldots, \varepsilon_{p(n)}\right)$ is a reduced path if $d_{\alpha}(\mathrm{P}(k-1), \mathrm{P}(k))=0$ for $1 \leqslant k \leqslant n$ and $\alpha \in \Pi$.

This definition of a reduced path is easily seen to be equivalent to that of [2, Section 2.3] . 

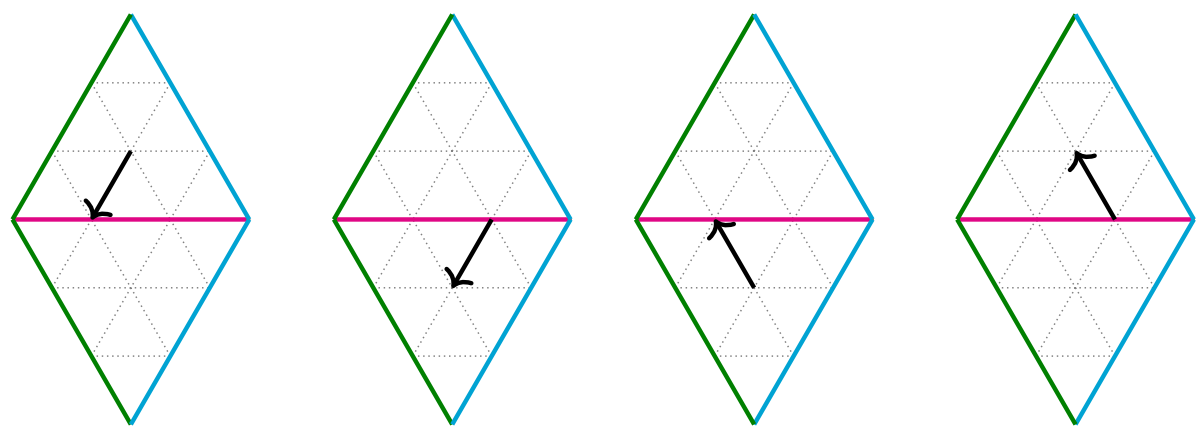

Fig. 12 The first and second paths have degrees -1 and +1 respectively. The third and fourth paths have degree 0 . Here we take the convention that the origin is below the pink hyperplane

There exist a unique reduced path in each -equivalence class (and, of course, each reduced path belongs to some $\sim$-equivalence class and so $\sim$-classes and reduced paths are in bijection). We remark that $\mathrm{T}_{\mu}$, the maximal path in the reverse cylindric ordering $\succ$, is an example of a reduced path. Given $S \in \operatorname{Path}_{\underline{h}}(n)$, we let $\min [S]$ denote the minimal path in the $\sim$-equivalence class containing $S$. Given a reduced path $\mathrm{P}_{\lambda} \in \operatorname{Path}_{\underline{h}}(\lambda)$, we have that

$$
\mathscr{H}_{n}^{\sigma} e_{\mathrm{P}_{\lambda}}=\mathbf{P}(\lambda) \oplus \bigoplus_{\mu \succ \lambda} k_{\mathrm{P}_{\lambda}}^{\mu} \mathbf{P}(\mu)
$$

decomposes (in a unitriangular fashion) as a sum of projective indecomposable modules for some generalised $p$-Kostka coefficients $k_{\mathrm{P}_{\lambda}}^{\mu} \in \mathbb{k}$. In general, we have

$$
\mathscr{H}_{n}^{\sigma} e_{\mathrm{P}_{\lambda}} ¥ \mathscr{H}_{n}^{\sigma} e_{\mathrm{Q}_{\lambda}}
$$

for reduced paths $\mathrm{P}_{\lambda}, \mathrm{Q}_{\lambda} \in \operatorname{Path}_{\underline{h}}(\lambda)$ and so the choice of reduced path does matter. (This is not surprising, the auxiliary steps in Soergel's algorithm for calculating Kazhdan-Lusztig polynomials produces a different pattern depending on the choice of reduced expression.) However, they do agree modulo higher terms under $»$, as we shall soon see (and indeed, after the cancellations in Soergel's algorithm one obtains that the Kazhdan-Lusztig polynomials are independent of choices of reduced expressions).

Lemma 3.4 Given $\lambda \in \mathscr{P}_{h}(n)$, let $\mathrm{P}_{\lambda}, Q_{\lambda}, \mathrm{S}_{\lambda}$ be any triple of reduced paths in $\operatorname{Path}_{\underline{h}}(\lambda)$. The element $e_{\mathrm{P}_{\lambda}}$ generates $\mathcal{H}^{ \pm \bar{\lambda}} / \mathcal{H}^{\succ \lambda}$ and moreover

$$
\psi_{Q_{\lambda}}^{\mathrm{P}_{\lambda}} \psi_{\mathrm{S}_{\lambda}}^{Q_{\lambda}}=k \psi_{\mathrm{S}_{\lambda}}^{\mathrm{P}_{\lambda}}+\mathcal{H}^{\succ \lambda}
$$

for some $k \in \mathbb{k} \backslash\{0\}$.

Proof Let $\mathrm{R}_{\lambda}$ be any reduced path in $\operatorname{Path}_{\underline{h}}(\lambda)$. Two paths have the same residue sequence if and only if they belong to the same $\sim$-class. If $S \sim R_{\lambda}$ then either $S=R_{\lambda}$ or $S$ terminates at a point $\mu \succ \lambda$. Thus, we have that

$$
e_{\mathrm{R}_{\lambda}} \mathbf{S}_{\mathbb{K}}(v) \neq 0 \text { implies } v \succcurlyeq \lambda \text { or } v=\lambda \text { and } e_{\mathrm{R}_{\lambda}} \mathbf{S}(\lambda)=e_{\mathrm{R}_{\lambda}} \mathbf{D}_{\mathbb{K}}(\lambda)=\psi_{\mathrm{T}_{\lambda}}^{\mathrm{R}_{\lambda}} .
$$

This implies that $e_{\mathrm{R}_{\lambda}} \in \mathcal{H}^{\succeq \lambda} / \mathcal{H}^{\succ \lambda}$ and therefore generates $\mathcal{H}^{\succeq \lambda} / \mathcal{H}^{\succ \lambda}$ and belongs to the simple head of the Specht module; the result follows. 
Definition 3.5 Given two paths

$$
\mathrm{P}=\left(\varepsilon_{i_{1}}, \varepsilon_{i_{2}}, \ldots, \varepsilon_{i_{p}}\right) \in \operatorname{Path}(\mu) \text { and } \mathrm{Q}=\left(\varepsilon_{j_{1}}, \varepsilon_{j_{2}}, \ldots, \varepsilon_{j_{q}}\right) \in \operatorname{Path}(v)
$$

we define the naive concatenated path

$$
\mathrm{P} \otimes \mathrm{Q}=\left(\varepsilon_{i_{1}}, \varepsilon_{i_{2}}, \ldots, \varepsilon_{i_{p}}, \varepsilon_{j_{1}}, \varepsilon_{j_{2}}, \ldots, \varepsilon_{j_{q}}\right) \in \operatorname{Path}(\mu+v) .
$$

\subsection{Branching coefficients}

We now discuss how one can think of a permutation as a morphism between pairs of paths in the alcove geometries of Sect. 3.1. Let $\lambda \in \mathcal{C}_{\underline{h}}(n)$. Given a pair of paths $S, T \in \operatorname{Path}(\lambda)$ we write the steps in $S$ and $T$ in sequence along the top and bottom edges of a frame, respectively. We can now reinterpret the element $w_{\top}^{S} \in \mathfrak{S}_{n}$ (of Sect. 2) as the unique step-preserving permutation with the minimal number of crossings.

In the following (running) example we label our paths by $\mathrm{P}_{\varnothing}\left(=\mathrm{T}_{\left(3^{3}\right)}\right)$ and $\mathrm{P}_{\alpha}^{\mathrm{b}}$. For this section, we do not need to know what inspires this notation; however, all will become clear in Sect. 4.

Example 3.6 We consider $\mathbb{k}_{\mathfrak{S}} \mathfrak{S}_{9}$ for $\mathbb{k}$ a field of characteristic 5; the characteristic is unimportant now, but inspires the notation and will be needed when we refer back to this example later. We set $\alpha=\varepsilon_{3}-\varepsilon_{1} \in \Pi$. Here we have

$$
\mathrm{P}_{\varnothing}=\left(\varepsilon_{1}, \varepsilon_{2}, \varepsilon_{3}, \varepsilon_{1}, \varepsilon_{2}, \varepsilon_{3}, \varepsilon_{1}, \varepsilon_{2}, \varepsilon_{3}\right) \quad \text { and } \mathrm{P}_{\alpha}^{b}=\left(\varepsilon_{1}, \varepsilon_{2}, \varepsilon_{1}, \varepsilon_{2}, \varepsilon_{1}, \varepsilon_{2}, \varepsilon_{3}, \varepsilon_{3}, \varepsilon_{3}\right)
$$

are two examples of paths of shape $\left(3^{3}\right)$. The unique step-preserving permutation of minimal length is given by

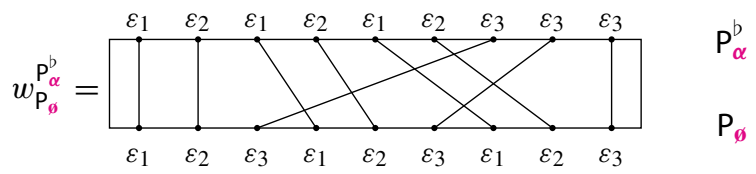

Notice that if two strands have the same step-label, then they do not cross. This is, of course, exactly what it means for a step-preserving permutation to be of minimal length.

Definition 3.7 Fix $(\mathrm{S}, \mathrm{T})$ an ordered pair of paths which both terminate at some point $\lambda \in$ $\mathscr{P}_{\underline{h}}(n)$. We now inductively construct a reduced expression for $w_{\mathrm{T}}^{\mathrm{S}}$. We define the branching coefficients

$$
d_{p}(\mathrm{~S}, \mathrm{~T})=w_{q}^{p} \quad \text { where } q=\left|\left\{1 \leqslant i \leqslant p \mid w_{\mathrm{T}}^{\mathrm{S}}(i) \leqslant w_{\mathrm{T}}^{\mathrm{S}}(p)\right\}\right|
$$

and

$$
\Upsilon_{p}(\mathrm{~S}, \mathrm{~T})=(-1)^{\sharp\left\{p<k \leqslant q \mid i_{k}=i_{p}\right\}} \psi_{d_{p}(\mathrm{~S}, \mathrm{~T})}
$$

for $1 \leqslant p \leqslant n$. These allow us to fix a distinguished reduced expression, $\underline{w}_{\top}^{\mathrm{S}}$, for $w_{\top}^{\mathrm{S}}$ as follows,

$$
\underline{w}_{\mathrm{T}}^{\mathrm{S}}=d_{1}(\mathrm{~S}, \mathrm{~T}) \ldots d_{n}(\mathrm{~S}, \mathrm{~T})
$$

and we set

$$
\Upsilon_{\mathrm{T}}^{\mathrm{S}}=e_{\mathrm{S}} \Upsilon_{\underline{w}_{\mathrm{T}}^{S}} e_{\mathrm{T}}=e_{\mathrm{S}} \Upsilon_{1}(\mathrm{~S}, \mathrm{~T}) \Upsilon_{2}(\mathrm{~S}, \mathrm{~T}) \cdots \Upsilon_{n}(\mathrm{~S}, \mathrm{~T}) e_{\mathrm{T}}
$$


Fig. 13 The reduced word, $\underline{w}_{\mathrm{P}_{\alpha}}^{\mathrm{P}_{\alpha}}$ (see also Examples 3.6 and 3.8)

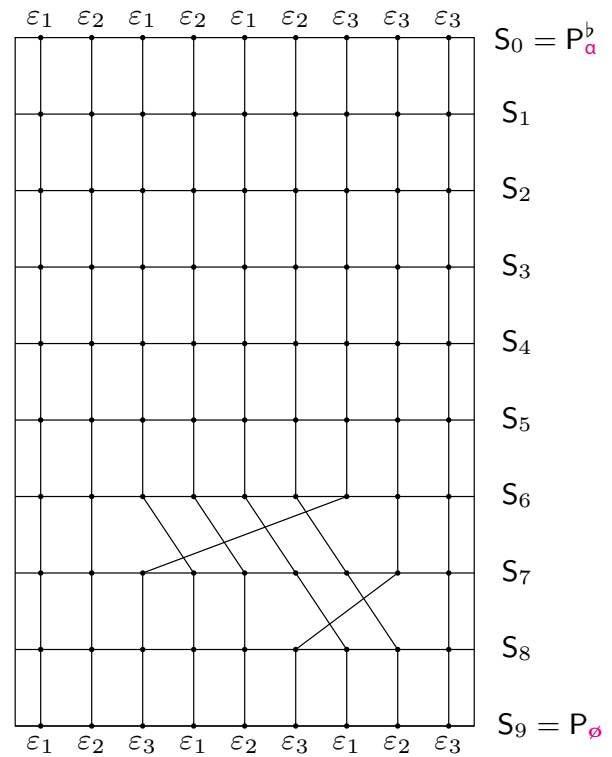

Example 3.8 We continue with the assumptions of Example 3.6. We have that

$$
1_{\mathfrak{S}_{9}}=d_{p}\left(\mathrm{P}_{\emptyset}, \mathrm{P}_{\alpha}^{b}\right)
$$

for each $p=1,2,3,4,5,6,9$ because $w_{\mathrm{P}_{\alpha}^{b}}^{\mathrm{P}_{\phi}}(p) \geqslant i$ for all $1 \leqslant i \leqslant p$. We have that

$$
d_{7}\left(\mathrm{P}_{\varnothing}, \mathrm{P}_{\alpha}^{\mathrm{b}}\right)=w_{3}^{7} \quad d_{8}\left(\mathrm{P}_{\varnothing}, \mathrm{P}_{\alpha}^{\mathrm{b}}\right)=w_{6}^{8}
$$

and so our reduced word is depicted in Fig. 13.

We can think of the branching coefficients as "one step morphisms" which allow us to mutate the path $\mathrm{S}$ into $\mathrm{T}$ via a series of $n$ steps (as each branching coefficient moves the position of one step in the path) and so this mutation proceeds via $n+1$ paths

$$
\mathrm{S}=\mathrm{S}_{0}, \mathrm{~S}_{1}, \mathrm{~S}_{2}, \ldots, \mathrm{S}_{n}=\mathrm{T}
$$

see Fig. 13 for an example. We now lift these branching coefficients to the KLR algebra.

Remark 3.9 The "sign twist" in Definition 3.7 is of no consequence in this paper as we are mostly concerned with constructing generators and bases of quiver Hecke algebras and their truncations. However, in order to match-up our relations with those of Elias-Williamson, this sign twist will be necessary and so we introduce it here for the purposes of consistency with [2].

Now, let's momentarily restrict our attention to pairs of paths of the form $\left(S, T_{\lambda}\right)$. In this case, the branching coefficients actually come from the "branching rule" for restriction along the tower $\cdots \subset \mathscr{H}_{n-1}^{\sigma} \subset \mathscr{H}_{n}^{\sigma} \subset \cdots$. To see this, we note that

$$
\underline{w}_{\mathrm{T}_{\lambda}}^{\mathrm{S}}=w_{1}\left(\mathrm{~S}, \mathrm{~T}_{\lambda}\right) w_{2}\left(\mathrm{~S}, \mathrm{~T}_{\lambda}\right) \ldots w_{n}\left(\mathrm{~S}, \mathrm{~T}_{\lambda}\right)
$$

where $w_{n}\left(\mathrm{~S}, \mathrm{~T}_{\lambda}\right)=w_{\mathrm{T}_{\lambda}(\square)}^{n}$ for some removable box $\square \in \operatorname{Rem}(\lambda)$ and where

$$
w_{1}\left(\mathrm{~S}, \mathrm{~T}_{\lambda}\right) w_{2}\left(\mathrm{~S}, \mathrm{~T}_{\lambda}\right) \ldots w_{n-1}\left(\mathrm{~S}, \mathrm{~T}_{\lambda}\right)=\underline{w}\left(\mathbf{S}_{\leqslant n-1}, \mathbf{T}_{\lambda-\square}\right) \in \mathfrak{S}_{n-1} \leqslant \mathfrak{S}_{n} .
$$


By Proposition 2.26, we have that

$$
\mathbf{S}_{\mathbb{k}}(\lambda-\square)\left\langle\operatorname{deg}\left(A_{r}\right)\right\rangle \cong \mathbb{k}\left\{\Upsilon_{\mathrm{T}_{\lambda}}^{\mathrm{S}} \mid \operatorname{Shape}\left(\mathrm{S}_{\leqslant n-1}\right)=\lambda-\square\right\} .
$$

Thus the branching coefficients above provide a factorisation of the cellular basis of Theorem 2.24 which is compatible with the restriction rule.

Example 3.10 Continuing with Examples 3.6 and 3.8, the lift of the path-morphism to the KLR algebra is as follows,

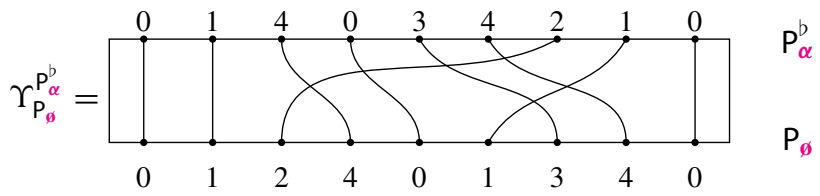

At each step in the restriction along the tower, there is precisely one removable box of any given residue and so the restriction is, in fact, a direct sum of Specht modules.

We wish to modify the branching coefficients above so that we can consider more general (families of) reduced paths $P_{\lambda}$ in place of the path $T_{\lambda}$. Given $S \in \operatorname{Path}_{\underline{h}}(\lambda)$, we can choose a reduced path vector as follows

$$
\underline{\mathrm{P}}_{\mathrm{S}}=\left(\mathrm{P}_{\mathrm{S}, 0}, \mathrm{P}_{\mathrm{S}, 1}, \ldots, \mathrm{P}_{\mathrm{S}, n}\right)
$$

such that Shape $\left(\mathrm{P}_{\mathrm{S}, k}\right)=\operatorname{Shape}\left(\mathrm{S}_{\leqslant k}\right)$ for each $0 \leqslant k \leqslant n$. In other words, we choose a reduced path $\mathrm{P}_{\mathrm{S}, p}$ for each and every point in the path $\mathrm{S}$. For $0 \leqslant p \leqslant n$ and $\operatorname{Shape}\left(\mathrm{S}_{<p}\right)+$ $\varepsilon_{i_{p}}=\operatorname{Shape}\left(\mathrm{S}_{\leqslant p}\right)$, we define the modified branching coefficient,

$$
d_{p}\left(\mathrm{~S}, \underline{\mathrm{P}}_{\mathrm{S}}\right)=\Upsilon_{\mathrm{P}_{\mathrm{S}, p}}^{\mathrm{P}_{\mathrm{S}-1} \otimes \mathrm{P}_{i p}}
$$

and we hence define

$$
\Upsilon_{\underline{\mathrm{P}}_{\mathrm{S}}}^{\mathrm{S}}=\prod_{1 \leqslant p \leqslant n} d_{p}\left(\mathrm{~S}, \underline{\mathrm{P}}_{\mathrm{S}}\right) .
$$

Here we have freely identified elements of algebras of different sizes using the usual embed$\operatorname{ding} \mathscr{H}_{n-1}^{\sigma} \hookrightarrow \mathscr{H}_{n}^{\sigma}$ given by $d \mapsto d \otimes\left(\sum_{i \in \mathbb{Z} / e \mathbb{Z}} e(i)\right)$. We set $\Upsilon_{\mathrm{S}}^{\underline{\mathrm{P}}_{\mathrm{S}}}:=\left(\Upsilon_{\underline{\mathrm{P}}_{\mathrm{S}}^{\mathrm{S}}}\right)^{*}$.

Remark 3.11 For symmetric groups there is a canonical choice of reduced path vector coming from the coset-like combinatorics which has historically been used for studying these groups. For the light leaves construction of Bott-Samelson endomorphism algebras, Libedinsky and Elias-Williamson require very different families of reduced path vectors whose origin can be seen as coming from a basis which can be written in terms of their 2-generators $[6,10]$.

Example 3.12 Continuing with Example 3.10 we have already noted that $\mathrm{P}_{\varnothing}=\mathrm{T}_{\left(3^{3}\right)}$. We choose to take the sequence $\mathrm{T}_{\mu}$ for $\mu=\operatorname{Shape}\left(\left(\mathrm{P}_{\alpha}^{b}\right)_{\leqslant k}\right)$ for $k \geqslant 0$ as our reduced path vector $\underline{P}_{S}$. Having made this choice, we have that $\Upsilon_{P_{S}}^{P_{\alpha}^{b}}=\Upsilon_{P_{\sigma}}^{P_{\alpha}^{b}}$ (this holds more generally, see the Corollary 3.14 and the discussion immediately prior). We record this in tableaux format to help the reader transition between the old and new ways of thinking.

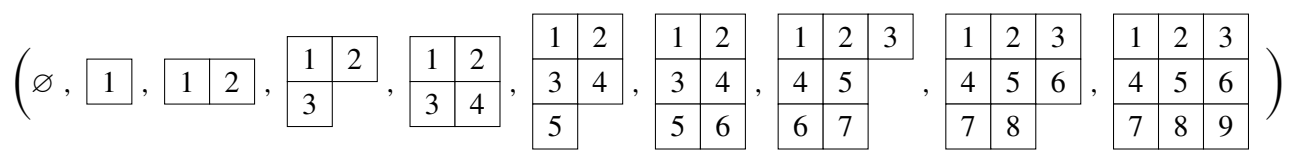


The light leaves basis will be given in terms of products $\Upsilon_{\underline{P}_{S}^{S}}^{S} \Upsilon_{T}^{\underline{P}_{T}}$ for $S, T \in \operatorname{Path}_{\underline{h}}(\lambda)$ and "compatible choices" of $\underline{\mathrm{P}}_{\mathrm{S}}$ and $\underline{\mathrm{P}}_{\mathrm{T}}$. Here the only condition for compatibility is that $\mathrm{P}_{\mathrm{S}, n}=\mathrm{Q}_{\lambda}=\mathrm{P}_{\mathrm{T}, n}$ for some fixed choice of reduced path $\mathrm{Q}_{\lambda} \in \operatorname{Path}_{\underline{h}}(\lambda)$, in other words the final choices of reduced path for each of $\mathrm{S}$ and $\mathrm{T}$ coincide. We remark that if $\mathrm{P}_{\mathrm{S}, n} \neq \mathrm{P}_{\mathrm{T}, n}$ then the product is clearly equal to zero (by idempotent considerations) and so this is the only sensible choice to make for such a product. In light of the above, we let $Q_{\lambda}$ be a reduced path and we say that a reduced path vector $\underline{\mathrm{P}}_{\mathrm{S}}$ terminates at $\mathrm{Q}_{\lambda}$ if $\underline{\mathrm{P}}_{\mathrm{S}, n}=\mathrm{Q}_{\lambda}$.

Theorem 3.13 (The light leaves basis) Let $\mathbb{k}$ be an integral domain and $\underline{h}=\left(h_{0}, \ldots, h_{\ell-1}\right) \in$ $\mathbb{N}^{\ell}$ be such that $h_{m} \leqslant \sigma_{m+1}-\sigma_{m}$ for $0 \leqslant m<\ell-1$ and $h_{\ell-1}<e+\sigma_{0}-\sigma_{\ell-1}$. For each $\lambda \in \mathscr{P}_{h}(n)$ we fix a reduced path $\mathrm{Q}_{\lambda} \in \operatorname{Path}_{h}(\lambda)$ and for each $\mathrm{S} \in \operatorname{Path}_{h}(\lambda)$, we fix an associated reduced path vector $\underline{\mathrm{P}}_{\mathrm{S}}$ terminating with $\mathrm{Q}_{\lambda}$. The $\mathbb{k}$-algebra $\mathscr{H}_{n}^{\sigma}$ is a graded cellular algebra with basis

$$
\left\{\Upsilon_{\underline{\mathrm{P}}_{\mathrm{S}}}^{\mathrm{S}} \Upsilon_{\mathrm{T}}^{\mathrm{P}_{\mathrm{T}}} \mid \mathrm{S}, \mathrm{T} \in \operatorname{Path}_{\underline{h}}(\lambda), \lambda \in \mathscr{P}_{\underline{h}}(n)\right\}
$$

anti-involution $*$ and the degree function $\operatorname{deg}: \operatorname{Path}_{\underline{h}} \rightarrow \mathbb{k}$.

Proof By Theorem 2.24, we have that

$$
\left\{\Upsilon_{T_{\lambda}}^{S} \Upsilon_{T}^{T_{\lambda}} \mid S, T \in \operatorname{Path}_{\underline{h}}(\lambda)\right\}
$$

provides a $\mathbb{k}$-basis of $\mathscr{H}^{\succeq \lambda} / \mathscr{H}^{\succ \lambda}$. By Lemma 3.4, we have that $\Upsilon_{\mathrm{Q}_{\lambda}}^{\mathrm{T}_{\lambda}} e_{\mathrm{Q}_{\lambda}} \Upsilon_{\mathrm{T}_{\lambda}}^{\mathrm{Q}_{\lambda}}=k e_{\mathrm{T}_{\lambda}}$ for some $k \in \mathbb{k} \backslash\{0\}$ modulo higher terms under $\succ$ and so

$$
\left\{\Upsilon_{\mathrm{T}_{\lambda}}^{\mathrm{S}}\left(\Upsilon_{\mathrm{Q}_{\lambda}}^{\mathrm{T}_{\lambda}} \Upsilon_{\mathrm{T}_{\lambda}}^{\mathrm{Q}_{\lambda}}\right) \Upsilon_{\mathrm{T}}^{\mathrm{T}_{\lambda}} \mid \mathrm{S}, \mathrm{T} \in \operatorname{Path}_{\underline{h}}(\lambda)\right\}
$$

provides a $\mathbb{k}$-basis of $\mathscr{H}^{\succeq \lambda} / \mathscr{H}^{\succ \lambda}$. By Eq. (3.3) and (3.4), we have that

$$
\left\{\Upsilon_{\mathrm{T}_{\lambda-\varepsilon_{i}}}^{\mathrm{s}} \Upsilon_{\mathrm{T}_{\lambda}}^{\mathrm{T}_{\lambda-\varepsilon_{i}} \otimes \mathrm{P}_{i}}\left(\Upsilon_{\mathrm{Q}_{\lambda}}^{\mathrm{T}_{\lambda}} \Upsilon_{\mathrm{T}_{\lambda}}^{\mathrm{Q}_{\lambda}}\right) \Upsilon_{\mathrm{T}}^{\mathrm{T}_{\lambda}} \mid \mathrm{s} \in \operatorname{Std}\left(\lambda-\varepsilon_{i}\right), \varepsilon_{i} \in \operatorname{Rem}(\lambda), \mathrm{T} \in \operatorname{Path}_{\underline{h}}(\lambda)\right\}
$$

provides a $\mathbb{k}$-basis of $\mathscr{H}^{\succeq \lambda} / \mathscr{H}^{\succ \lambda}$. By Proposition 2.26, we have that

$$
\Upsilon_{\mathrm{T}_{\lambda}}^{\mathrm{T}_{\lambda-\varepsilon_{i}} \otimes \mathrm{P}_{i}} e_{\mathrm{T}_{\lambda}}
$$

generates a left subquotient of $\mathscr{H}^{\succeq \lambda} / \mathscr{H}^{\succ \lambda}$ which is isomorphic to $\mathbf{S}_{\mathbb{k}}\left(\lambda-\varepsilon_{i}\right)$. Now, for each pair $\varepsilon_{i} \in \operatorname{Rem}(\lambda)$ and $\mathrm{s} \in \operatorname{Std}\left(\lambda-\varepsilon_{i}\right)$, we fix a corresponding choice of reduced path $\mathrm{P}_{\mathrm{S}, n-1} \in \operatorname{Path}_{\underline{h}}\left(\lambda-\varepsilon_{i}\right)$. By Lemma 3.4 and Proposition 2.26, we have that the set of all

$$
\Upsilon_{\mathrm{T}_{\lambda-\varepsilon_{i}}}^{\mathrm{S}}\left(\Upsilon_{\mathrm{P}_{\mathrm{S}, n-1} \otimes \mathrm{P}_{i}}^{\mathrm{T}_{\lambda-\varepsilon_{i}} \otimes \mathrm{P}_{i}} \Upsilon_{\mathrm{T}_{\lambda-\varepsilon_{i}} \otimes \mathrm{P}_{i}}^{\mathrm{P}_{\mathrm{S}, n-1} \otimes \mathrm{P}_{i}}\right) \Upsilon_{\mathrm{T}_{\lambda}}^{\mathrm{T}_{\lambda-\varepsilon_{i}} \otimes \mathrm{P}_{i}}\left(\Upsilon_{\mathrm{Q}_{\lambda}}^{\mathrm{T}_{\lambda}} \Upsilon_{\mathrm{T}_{\lambda}}^{\mathrm{Q}_{\lambda}}\right) \Upsilon_{\mathrm{T}}^{\mathrm{T}_{\lambda}}
$$

as we vary over all $\mathrm{s} \in \operatorname{Path}_{\underline{h}}\left(\lambda-\varepsilon_{i}\right), \varepsilon_{i} \in \operatorname{Rem}(\lambda)$, and $\mathrm{T} \in \operatorname{Path}_{\underline{h}}(\lambda)$ provides a $\mathbb{k}$-basis of $\mathscr{H}^{\geq \lambda} / \mathscr{H}^{\succ \lambda}$. Re-bracketing the above, we have that the set of all

$$
\left(\Upsilon_{\mathrm{T}_{\lambda-\varepsilon_{i}}}^{\mathrm{S}} \Upsilon_{\mathrm{P}_{\mathrm{S}, n-1}}^{\mathrm{T}_{\lambda-\varepsilon_{i}}}\right)\left(\Upsilon_{\mathrm{T}_{\lambda-\varepsilon_{i}} \otimes \mathrm{P}_{i}}^{\mathrm{P}_{\mathrm{S}, n-1} \otimes \mathrm{P}_{i}} \Upsilon_{\mathrm{T}_{\lambda}}^{\mathrm{T}_{\lambda-\varepsilon_{i}} \otimes \mathrm{P}_{i}} \Upsilon_{\mathrm{Q}_{\lambda}}^{\mathrm{T}_{\lambda}}\right)\left(\Upsilon_{\mathrm{T}_{\lambda}}^{\mathrm{Q}_{\lambda}} \Upsilon_{\mathrm{T}}^{\mathrm{T}_{\lambda}}\right)
$$

as we vary over all $\mathrm{s} \in \operatorname{Path}_{\underline{h}}\left(\lambda-\varepsilon_{i}\right), \varepsilon_{i} \in \operatorname{Rem}(\lambda)$, and $\mathrm{T} \in \operatorname{Path}_{\underline{h}}(\lambda)$ provides a $\mathbb{k}$-basis of $\mathscr{H}^{\succeq \lambda} / \mathscr{H}^{\succ \lambda}$. Finally, simplifying using Proposition 2.22 we obtain that

$$
\left\{\Upsilon_{\mathrm{P}_{\mathrm{S}, n-1}^{\mathrm{s}}}^{\mathrm{s}} \Upsilon_{\mathrm{Q}_{\lambda}}^{\mathrm{P}_{\mathrm{S}, n-1} \otimes \mathrm{P}_{i}} \Upsilon_{\mathrm{T}}^{\mathrm{Q}_{\lambda}} \mid \mathrm{s} \otimes \mathrm{P}_{i} \in \operatorname{Path}_{\underline{h}}\left(\lambda-\varepsilon_{i}\right), \varepsilon_{i} \in \operatorname{Rem}(\lambda), \mathrm{T} \in \operatorname{Path}_{\underline{h}}(\lambda)\right\}
$$


is a $\mathbb{k}$-basis of $\mathscr{H}^{\geq \lambda} / \mathscr{H}^{\succ \lambda}$ where we note that the middle term in the KLR-product is our modified branching coefficient. Repeating $n$ times, we have that

$$
\left\{\Upsilon_{\mathrm{P}_{\mathrm{S}, 1}}^{\mathrm{P}_{\mathrm{S}, 0} \otimes \mathrm{P}_{i_{1}}} \ldots \Upsilon_{\mathrm{P}_{\mathrm{S}, n-1}}^{\mathrm{P}_{\mathrm{S}, n-2} \otimes \mathrm{P}_{i_{n-1}}} \Upsilon_{\mathrm{Q}_{\lambda}}^{\mathrm{P}_{\mathrm{S}, n-1} \otimes \mathrm{P}_{i_{n}}} \Upsilon_{\mathrm{T}}^{\mathrm{Q}_{\lambda}} \mid \mathrm{S}, \mathrm{T} \in \operatorname{Path}_{\underline{h}}(\lambda)\right\}
$$

is a $\mathbb{k}$-basis of $\mathscr{H}^{\succeq \lambda} / \mathscr{H}^{\succ \lambda}$; repeating the above for the righthand-side, the result follows.

In particular, we can set $\underline{\mathrm{P}}_{\mathrm{S}}=\left(\mathrm{Q}_{\lambda \downarrow} \downarrow_{k}\right)_{k \geqslant 0}$ and obtain the following corollary, which specialises to Theorem 2.24 for $\mathrm{Q}_{\lambda}=\mathrm{T}_{\lambda}$.

Corollary 3.14 For each $\lambda \in \mathscr{P}_{\underline{h}}(n)$ we fix a reduced path $\mathrm{Q}_{\lambda} \in \operatorname{Path}_{\underline{h}}(\lambda)$. The $\mathbb{k}$-algebra $\mathscr{H}_{n}^{\sigma}$ is a graded cellular algebra with basis

$$
\left\{\Upsilon_{Q_{\lambda}}^{S} \Upsilon_{\mathrm{T}}^{Q_{\lambda}} \mid \mathrm{S}, \mathrm{T} \in \operatorname{Path}_{\underline{h}}(\lambda), \lambda \in \mathscr{P}_{\underline{h}}(n)\right\}
$$

anti-involution $*$ and the degree function $\operatorname{deg}: \operatorname{Path}_{\underline{h}} \rightarrow \mathbb{Z}$.

\section{Light leaf generators for the principal block}

We now restrict our attention to the principal block and illustrate how the constructions of previous sections specialise to be familiar ideas from Soergel diagrammatics. In particular, we provide an exact analogue of Libedinsky's and Elias-Williamson's algorithmic construction of a light leaves basis for such blocks. In order to do this, we provide a short list of pathmorphisms which we will show generate the algebra $\mathrm{f}_{n, \sigma}\left(\mathcal{H}_{n}^{\sigma} / \mathcal{H}_{n}^{\sigma} \mathrm{y}_{\underline{h}} \mathcal{H}_{n}^{\sigma}\right) \mathrm{f}_{n, \sigma}$ (thus proving Theorem B).

\subsection{Alcove paths}

When passing from multicompositions to our geometry $\overline{\mathbb{E}}_{h, l}$, many non-trivial elements map to the origin. One such element is $\delta=\left(\left(h_{1}\right), \ldots,\left(h_{\ell}\right)\right) \in \mathscr{P}_{\underline{h}}(h)$. (Recall our transpose convention for embedding multipartitions into our geometry.) We will sometimes refer to this as the determinant as (for the symmetric group) it corresponds to the determinant representation of the associated general linear group. We will also need to consider elements corresponding to powers of the determinant, namely $\delta_{k}=\left(\left(h_{1}^{k}\right), \ldots,\left(h_{\ell}^{k}\right)\right) \in \mathscr{P}_{\underline{h}}(k h)$. We now restrict our attention to paths between points in the principal linkage class, in other words to paths between points in $\widehat{\mathfrak{S}}_{h} \cdot 0$. Such points can be represented by multicompositions $\mu$ in $\widehat{\mathfrak{S}}_{h} \cdot \delta_{k}$ for some choice of $k$.

Definition 4.1 We will associate alcove paths to certain words in the alphabet

$$
S \cup\{1\}=\left\{s_{\alpha} \mid \alpha \in \Pi \cup\{\emptyset\}\right\}
$$

where $s_{\emptyset}=1$. That is, we will consider words in the generators of the affine Weyl group, but enriched with explicit occurrences of the identity in these expressions. We refer to the number of elements in such an expression (including the occurrences of the identity) as the degree of this expression. We say that an enriched word is reduced if, upon forgetting occurrences of the identity in the expression, the resulting word is reduced.

Given a path $\mathrm{P}$ between points in the principal linkage class, the end point lies in the interior of an alcove of the form $w A_{0}$ for some $w \in \widehat{\mathfrak{S}}_{h}$. If we write $w$ as a word in our 
alphabet, and then replace each element $s_{\alpha}$ by the corresponding non-affine reflection $s_{\alpha}$ in $\mathfrak{S}_{h}$ to form the element $\bar{w} \in \mathfrak{S}_{h}$ then the basis vectors $\varepsilon_{i}$ are permuted by the corresponding action of $\bar{w}$ to give $\varepsilon_{\bar{w}(i)}$, and there is an isomorphism from $\overline{\mathbb{E}}_{h, l}$ to itself which maps $A_{0}$ to $w A_{0}$ such that 0 maps to $w \cdot 0$, coloured walls map to walls of the same colour, and each basis element $\varepsilon_{i}$ map to $\varepsilon \bar{w}(i)$. Under this map we can transform a path Q starting at the origin to a path starting at $w \cdot 0$ which passes through the same sequence of coloured walls as $\mathrm{Q}$ does.

Definition 4.2 Given two paths $\mathrm{P}=\left(\varepsilon_{i_{1}}, \varepsilon_{i_{2}}, \ldots, \varepsilon_{i_{p}}\right) \in \operatorname{Path}(\mu)$ and $\mathrm{Q}=\left(\varepsilon_{j_{1}}, \varepsilon_{j_{2}}, \ldots, \varepsilon_{j_{q}}\right)$ $\in \operatorname{Path}(v)$ with the endpoint of $\mathrm{P}$ lying in the closure of some alcove $w A_{0}$ we define the contextualised concatenated path

$$
\mathrm{P} \otimes_{w} \mathrm{Q}=\left(\varepsilon_{i_{1}}, \varepsilon_{i_{2}}, \ldots, \varepsilon_{i_{p}}\right) \otimes\left(\varepsilon_{\bar{w}\left(j_{1}\right)}, \varepsilon_{\bar{w}\left(j_{2}\right)}, \ldots, \varepsilon_{\bar{w}\left(j_{q}\right)}\right) \in \operatorname{Path}(\mu+(w \cdot v)) .
$$

If there is a unique such $w$ then we may simply write $\mathrm{P} \otimes \mathrm{Q}$. If $w=s_{\alpha}$ we will simply write $\mathrm{P} \otimes_{\alpha} \mathrm{Q}$.

We now define the building blocks from which all of our distinguished paths will be constructed. We begin by defining certain integers that describe the position of the origin in our fundamental alcove.

Definition 4.3 Given $\alpha \in \Pi$ we define $b_{\alpha}$ to be the distance from the origin to the wall corresponding to $\alpha$, and let $b_{\emptyset}=1$. Given our earlier conventions this corresponds to setting

$$
b_{\varepsilon_{H_{m}+i}-\varepsilon_{H_{m}+i+1}}=1
$$

for $1 \leqslant i<h_{m+1}$ and $0 \leqslant m<\ell$ and that

$$
b_{\varepsilon_{H_{m}}-\varepsilon_{H_{m}+1}}=\sigma_{m+1}-\sigma_{m}-h_{m}+1 \quad b_{\varepsilon_{h}-\varepsilon_{1}}=e+\sigma_{0}-\sigma_{\ell-1}-h_{\ell-1}+1
$$

for $0 \leqslant m<\ell-1$. We sometimes write $\delta_{\alpha}$ for the element $\delta_{b_{\alpha}}$. Given $\alpha, \beta \in \Pi$ we set $b_{\alpha \beta}=b_{\alpha}+b_{\beta}$.

Example 4.4 Let $e=5, h=3$ and $\ell=1$ as in Fig. 11. Then $b_{\varepsilon_{2}-\varepsilon_{3}}$ and $b_{\varepsilon_{1}-\varepsilon_{2}}$ both equal 1 , while $b_{\varepsilon_{3}-\varepsilon_{1}}=3$ and $b_{\emptyset}=1$.

Example 4.5 Let $e=7, h=2$ and $\ell=2$ and $\sigma=(0,3) \in \mathbb{Z}^{2}$. Then $b_{\varepsilon_{1}-\varepsilon_{2}}$ and $b_{\varepsilon_{3}-\varepsilon_{4}}$ both equal 1 , while $b_{\varepsilon_{4}-\varepsilon_{1}}=3, b_{\varepsilon_{2}-\varepsilon_{3}}=2$, and $b_{\emptyset}=1$.

We can now define our basic building blocks for paths.

Definition 4.6 Given $\alpha=\varepsilon_{i}-\varepsilon_{i+1} \in \Pi$, we consider the multicomposition $s_{\alpha} \cdot \delta_{\alpha}$ with all columns of length $b_{\alpha}$, with the exception of the $i$ th and $(i+1)$ st columns, which are of length 0 and $2 b_{\alpha}$, respectively. We set

$$
\mathrm{M}_{i}=\left(\varepsilon_{1}, \ldots, \varepsilon_{i-1}, \widehat{\varepsilon_{i}}, \varepsilon_{i+1}, \ldots, \varepsilon_{h}\right) \text { and } \mathrm{P}_{i}=\left(+\varepsilon_{i}\right)
$$

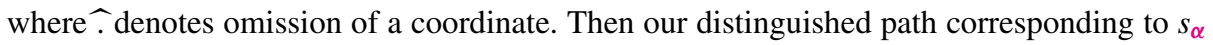
is given by

$$
\mathrm{P}_{\alpha}=\mathrm{M}_{i}^{b_{\alpha}} \otimes \mathrm{P}_{i+1}^{b_{\alpha}} \in \operatorname{Path}\left(s_{\alpha} \cdot \delta_{\alpha}\right) .
$$

The distinguished path corresponding to $\emptyset$ is given by

$$
\mathrm{P}_{\emptyset}=\left(\varepsilon_{1}, \ldots, \varepsilon_{i-1}, \varepsilon_{i}, \varepsilon_{i+1}, \ldots, \varepsilon_{h}\right) \in \operatorname{Path}(\delta)=\operatorname{Path}\left(s_{\emptyset} \cdot \delta\right)
$$

and set $\mathrm{P}_{\emptyset}=\left(\mathrm{P}_{\emptyset}\right)^{b_{\alpha}}$. 
Fig. 14 The leftmost two diagrams picture the path $\mathrm{P}_{\alpha}$ walking through an $\alpha$-hyperplane in $\overline{\mathbb{E}}_{1,3}^{+}$, and the path $\mathrm{P}_{\alpha}^{b}$ which reflects this path through the same $\alpha$-hyperplane. The rightmost diagram pictures the path $\mathrm{P}_{\emptyset}$ in $\overline{\mathbb{E}}_{1,3}^{+}$. We have bent the paths slightly to make them clearer
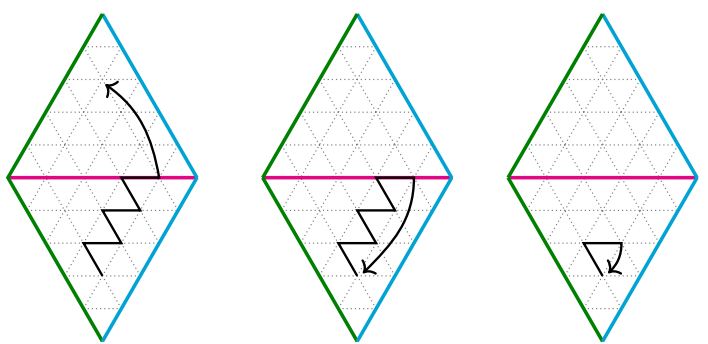

Given all of the above, we can finally define our distinguished paths for general words in our alphabet. There will be one such path for each word in our alphabet, and they will be defined by induction on the degree of the word, as follows.

Definition 4.7 We now define a distinguished path $\mathrm{P}_{\underline{w}}$ for each word $\underline{w}$ in our alphabet $S \cup\{1\}$ by induction on the degree of $\underline{w}$. If $\underline{w}$ is $s_{\emptyset}$ or a simple reflection $s_{\alpha}$ we have already defined the distinguished path in Definition 4.6. Otherwise if $\underline{w}=s_{\alpha} \underline{w}^{\prime}$ then we define

$$
\mathrm{P}_{\underline{w}}:=\mathrm{P}_{\alpha} \otimes_{\alpha} \mathrm{P}_{\underline{w^{\prime}}} .
$$

If $\underline{w}$ is a reduced word in $\widehat{\mathfrak{S}}_{h l}$, then the corresponding path $\mathrm{P}_{\underline{w}}$ is a reduced path.

Remark 4.8 Contextualised concatenation is not associative (if we wish to decorate the tensor products with the corresponding elements $w$ ). As we will typically be constructing paths as in Definition 4.7 we will adopt the convention that an unbracketed concatenation of $n$ terms corresponds to bracketing from the right:

$$
\mathrm{Q}_{1} \otimes \mathrm{Q}_{2} \otimes \mathrm{Q}_{3} \otimes \cdots \mathrm{Q}_{n}=\mathrm{Q}_{1} \otimes\left(\mathrm{Q}_{2} \otimes\left(\mathrm{Q}_{3} \otimes\left(\cdots \otimes \mathrm{Q}_{n}\right) \cdots\right)\right) .
$$

We will also need certain reflections of our distinguished paths corresponding to elements of $\Pi$.

Definition 4.9 Given $\alpha \in \Pi$ we set

$$
\mathrm{P}_{\alpha}^{b}=\mathrm{M}_{i}^{b_{\alpha}} \otimes \mathrm{P}_{i}^{b_{\alpha}}=\mathrm{M}_{i}^{b_{\alpha}} \otimes_{\alpha} \mathrm{P}_{i+1}^{b_{\alpha}}=\left(+\varepsilon_{1}, \ldots,+\varepsilon_{i-1}, \widehat{+\varepsilon_{i}},+\varepsilon_{i+1}, \ldots,+\varepsilon_{h}\right)^{b_{\alpha}} \otimes\left(\varepsilon_{i}\right)^{b_{\alpha}}
$$

the path obtained by reflecting the second part of $\mathrm{P}_{\alpha}$ in the wall through which it passes.

Example 4.10 We illustrate these various constructions in a series of examples. In the first two diagrams of Fig. 14, we illustrate the basic path $\mathrm{P}_{\alpha}$ and the path $\mathrm{P}_{\alpha}^{b}$ and in the rightmost diagram of Fig. 14, we illustrate the path $\mathrm{P}_{\emptyset}$. A more complicated example is illustrated in Fig. 11 , where we show the distinguished path $\mathrm{P}_{\underline{w}}$ for $\underline{w}=\boldsymbol{s}_{\varepsilon_{3}-\varepsilon_{1}} s_{\varepsilon_{2}-\varepsilon_{1}} \boldsymbol{s}_{\varepsilon_{3}-\varepsilon_{2}} s_{\varepsilon_{3}-\varepsilon_{1}} \boldsymbol{s}_{\varepsilon_{2}-\varepsilon_{1}} \boldsymbol{s}_{\varepsilon_{3}}-\varepsilon_{2}$ as in Fig. 11. The components of the path between consecutive black nodes correspond to individual $\mathrm{P}_{\alpha} \mathrm{s}$.

\subsection{The principal block of $\mathscr{H}_{n}^{\sigma}$}

We now restrict our attention to regular blocks of $\mathscr{H}_{n}^{\sigma}$. In order to do this, we first recall that we consider an element of the quiver Hecke algebra to be a morphism between paths. The easiest elements to construct are the idempotents corresponding to the trivial morphism from 
a path to itself. Given $\alpha$ a simple reflection or $\alpha=\emptyset$, we have an associated path $\mathrm{P}_{\alpha}$, a trivial bijection $w_{\mathrm{P}_{\alpha}}^{\mathrm{P}_{\alpha}}=1 \in \mathfrak{S}_{b_{\alpha} h}$, and an idempotent element of the quiver Hecke algebra

$$
e_{\mathrm{P}_{\alpha}}:=e_{\mathrm{res}\left(\mathrm{P}_{\alpha}\right)} \in \mathcal{H}_{b_{\alpha}}^{\sigma} h
$$

More generally, given any $\underline{w}=s_{\alpha^{(1)}} s_{\alpha^{(2)}} \ldots s_{\alpha^{(k)}}$, we have an associated path $\mathrm{P}_{\underline{w}}$, and an element of the quiver Hecke algebra

$$
e_{\mathrm{P}_{\underline{w}}}:=e_{\mathrm{res}\left(\mathrm{P}_{\underline{w}}\right)}=e_{\mathrm{P}_{\alpha}(1)} \otimes e_{\mathrm{P}_{\alpha}(2)} \otimes \cdots \otimes e_{\mathrm{P}_{\alpha}(k)} \in \mathscr{H}_{h b_{\alpha^{(1)}}+\cdots+h b_{\alpha^{(k)}}}^{\sigma}
$$

We let $\operatorname{Std}_{n, \sigma}(\lambda)$ be the set of all standard $\lambda$-tableaux which can be obtained by contextualised concatenation of paths from the set

$$
\left\{\mathrm{P}_{\alpha} \mid \alpha \in \Pi\right\} \cup\left\{\mathrm{P}_{\alpha}^{b} \mid \alpha \in \Pi\right\} \cup\left\{\mathrm{P}_{\emptyset}\right\} .
$$

We let $\mathscr{P}_{\underline{h}}(n, \sigma)=\left\{\lambda \in \mathscr{P}_{\underline{h}}(n) \mid \operatorname{Std}_{n, \sigma}(\lambda) \neq \emptyset\right\}$. We let $\operatorname{Std}_{n, \sigma}=\cup_{\lambda \in \mathscr{P}_{h}(n, \sigma)} \operatorname{Std}_{n, \sigma}(\lambda)$. For example, the path in Fig. 11 is equal to $\mathrm{P}_{\alpha} \otimes \mathrm{P}_{\gamma} \otimes \mathrm{P}_{\beta} \otimes \mathrm{P}_{\alpha} \otimes \mathrm{P}_{\gamma} \otimes \mathrm{P}_{\beta}$. We define

$$
\mathrm{f}_{n, \sigma}=\sum_{\substack{\mathrm{S} \in \operatorname{Std}_{n, \sigma}(\lambda) \\ \lambda \in \mathscr{P}_{h}(n, \sigma)}} e_{\mathrm{S}}
$$

and the remainder of this paper will be dedicated to understanding the algebra

$$
\mathrm{f}_{n, \sigma}\left(\mathcal{H}_{n}^{\sigma} / \mathcal{H}_{n}^{\sigma} \mathrm{y}_{\underline{h}} \mathcal{H}_{n}^{\sigma}\right) \mathrm{f}_{n, \sigma}
$$

In fact, we will provide a concise list of generators for this truncated algebra (in the spirit of [6]) and rewrite the basis of Theorem 3.13 in terms of these generators.

In this section, we use our concrete branching coefficients to define the "Soergel 2generators" of $\mathrm{f}_{n, \sigma}\left(\mathcal{H}_{n}^{\sigma} / \mathcal{H}_{n}^{\sigma} \mathrm{y}_{h} \mathcal{H}_{n}^{\sigma}\right) \mathrm{f}_{n, \sigma}$ explicitly. In the companion paper [2], we will show that these generators are actually independent of these choices of reduced expressions (however, this won't be needed here-we simply make a note, again, for purposes of consistency with [2]).

\subsection{Generator morphisms in degree zero}

We first discuss how to pass between paths $\mathrm{P}_{\underline{w}}$ and $\mathrm{P}_{\underline{w^{\prime}}}$ which are in different linkage classes but for which $\underline{w}$ and $\underline{w}^{\prime}$ have the same underlying permutation. Fix two such paths

$\mathrm{P}_{\underline{w}}=\mathrm{P}_{\alpha^{(1)}} \otimes \mathrm{P}_{\alpha^{(2)}} \otimes \cdots \otimes \mathrm{P}_{\alpha^{(k)}} \in \mathrm{Path}_{\underline{h}}(\lambda) \quad \mathrm{P}_{\underline{w^{\prime}}}=\mathrm{P}_{\beta^{(1)}} \otimes \mathrm{P}_{\beta^{(2)}} \otimes \cdots \otimes \mathrm{P}_{\beta^{(k)}} \in \mathrm{Path}_{\underline{h}}(\lambda)$

with $\alpha^{(1)}, \ldots, \alpha^{(k)}, \beta^{(1)}, \ldots, \beta^{(k)} \in \Pi \cup\{\emptyset\}$. We suppose, only for the purposes of this motivational discussion, that both paths are reduced. In which case, we have that $w \in \widehat{\mathfrak{S}}_{h}$ and so the expressions $\underline{w}$ and $\underline{w}^{\prime}$ differ only by applying Coxeter relations in of $\widehat{\mathfrak{S}}_{h}$ and the trivial "adjustment" relation $s_{i} 1=1 s_{i}$ (made necessary by our augmentation of the Coxeter presentation). Moreover, $\underline{w}$ and $\underline{w}^{\prime}$ are both reduced expressions and so we need only apply the "hexagon" relation $s_{i} s_{i+1} s_{i}=s_{i+1} s_{i} s_{i+1}$ and the "commutation" relation $s_{i} s_{j}=s_{j} s_{i}$ for $|i-j|>1$. The remainder of this subsection will be dedicated to lifting these pathmorphisms to the level of generators of the KLR algebra. We stress that one can apply these adjustment/hexagon/commutator path-morphisms to any paths (not just reduced paths) but the reduced paths provide the motivation. 
Fig. 15 We let $h=3, \ell=1$, $e=5$ and $\alpha=\varepsilon_{3}-\varepsilon_{1}$. The adjustment term adj $\operatorname{ad}_{\alpha \emptyset}^{\emptyset \alpha}$ is illustrated

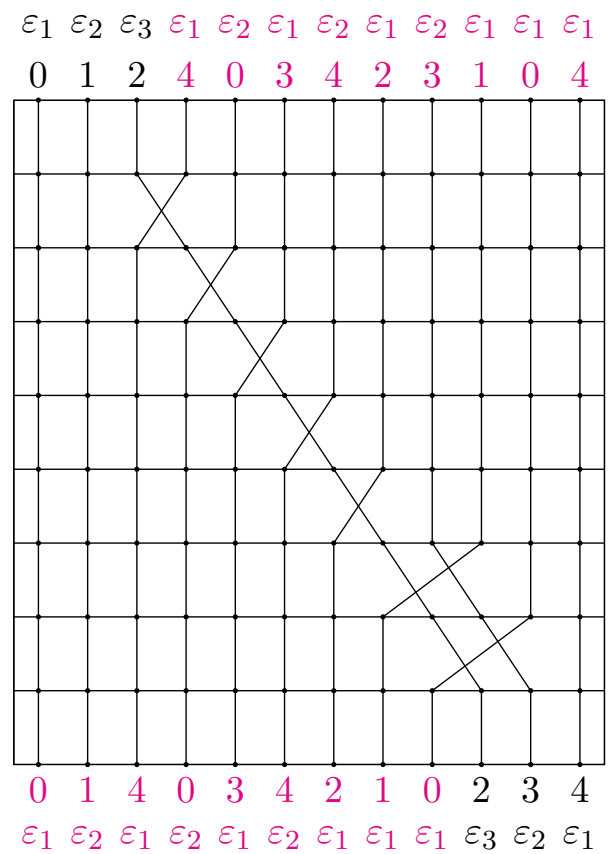

Fig. 16 We let $h=3, \ell=1$, $e=5$ and $\alpha=\varepsilon_{3}-\varepsilon_{1}$. We picture the paths $\mathrm{P}_{\alpha \emptyset}$ and $\mathrm{P}_{\emptyset \alpha}$.
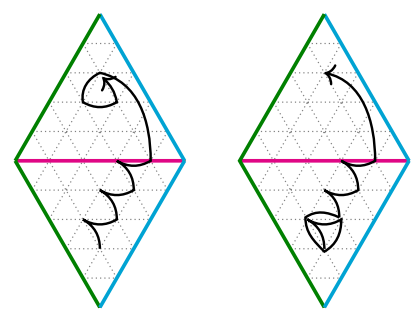

\subsubsection{Adjustment generator}

We will refer to the passage between alcove paths which differ only by occurrences of $s_{\emptyset}=1$ (and their associated idempotents) as "adjustment". We define the KLR-adjustment generator to be the element

$$
\operatorname{adj}_{\emptyset \alpha}^{\alpha \emptyset}:=\Upsilon_{P_{\emptyset \alpha}}^{P_{\alpha \emptyset}} \text {. }
$$

Examples of the paths $\mathrm{P}_{\alpha \emptyset}, \mathrm{P}_{\emptyset \alpha}$, and adjustment generators are given in Figs. 15 and 16.

\subsubsection{The KLR hexagon diagram}

We wish to pass between the two distinct paths around a vertex in our alcove geometry which lies at the intersection of two hyperplanes labelled by non-commuting reflections. To this end, we let $\alpha, \beta \in \Pi$ label a pair of non-commuting reflections. Of course, one path around the vertex may be longer than the other. Thus, we have two cases to consider: if $b_{\alpha} \geqslant b_{\beta}$ then we must pass between the paths $\mathrm{P}_{\alpha \beta \alpha}$ and $\mathrm{P}_{\varnothing-\varnothing} \otimes \mathrm{P}_{\beta \alpha \beta}$ and if $b_{\alpha} \leqslant b_{\beta}$ then we pass between the paths $\mathrm{P}_{\emptyset-\varnothing} \otimes \mathrm{P}_{\alpha \beta \alpha}$ and $\mathrm{P}_{\beta \alpha \beta}$, where here $\emptyset-\emptyset:=\emptyset^{b_{\alpha}-b_{\beta}}$. 

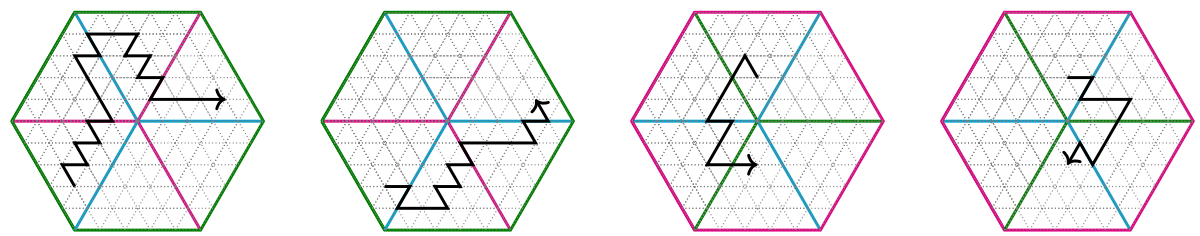

Fig. 17 We let $h=3, \ell=1, e=5$ and $\alpha=\varepsilon_{3}-\varepsilon_{1}$ and $\beta=\varepsilon_{1}-\varepsilon_{2}$ and $\gamma=\varepsilon_{2}-\varepsilon_{3}$. The paths $\mathrm{P}_{\alpha \beta \alpha}$, $\mathrm{P}_{\beta \alpha \beta}, \mathrm{P}_{\gamma \beta \gamma}$ and $\mathrm{P}_{\beta \gamma \beta}$ are pictured

Fig. 18 We let $h=3, \ell=1$, $e=5$ and $\beta=\varepsilon_{1}-\varepsilon_{2}$ and $\gamma=\varepsilon_{2}-\varepsilon_{3}$. We picture $\operatorname{hex}_{\mathbf{P}_{\gamma \beta \gamma}}^{\mathrm{P}_{\beta \gamma \beta}}$

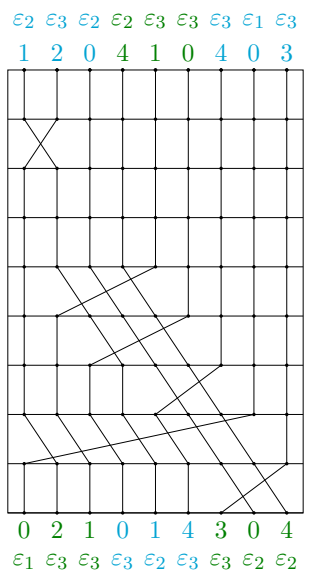

Fig. 19 We let $h=1, \ell=4$, $\kappa=(0,2,4,6) \in(\mathbb{Z} / 8 \mathbb{Z})^{4}$ and $\beta=\varepsilon_{1}-\varepsilon_{2}$ and $\gamma=\varepsilon_{3}-\varepsilon_{4}$. We picture the element $\operatorname{com}_{\gamma \beta}^{\beta \gamma}$, the corresponding paths are
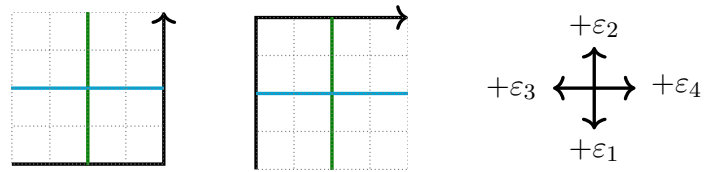
depicted in Fig. 20

We define the KLR-hexagon to be the element

$$
\operatorname{hex}_{\beta \alpha \beta}^{\alpha \beta \alpha}:=\Upsilon_{\mathrm{P}_{\phi \beta-\phi} \otimes \mathrm{P}_{\beta \alpha \beta}}^{\mathrm{P}_{\alpha \beta \alpha}} \quad \text { or } \quad \operatorname{hex}_{\beta \alpha \beta}^{\alpha \beta \alpha}:=\Upsilon_{\mathrm{P}_{\beta \alpha \beta}}^{\mathrm{P}_{\phi-\phi} \otimes \mathrm{P}_{\alpha \beta \alpha}}
$$

for $b_{\alpha} \geqslant b_{\beta}$ or $b_{\alpha} \leqslant b_{\beta}$ respectively. Two such pairs of paths are despited in Fig. 17. For the latter pair, the corresponding KLR-hexagon element is depicted in Fig. 18.

\subsubsection{The KLR commutator}

Let $\gamma, \beta \in \Pi$ be roots labelling commuting reflections. We wish to understand the morphism relating the paths $\mathrm{P}_{\gamma} \otimes \mathrm{P}_{\beta}$ to $\mathrm{P}_{\beta} \otimes \mathrm{P}_{\gamma}$. We define the KLR-commutator to be the element

$$
\operatorname{com}_{\beta \gamma}^{\gamma \beta}:=\Upsilon_{\mathrm{P}_{\beta} \otimes \mathrm{P}_{\gamma}}^{\mathrm{P}_{\gamma} \otimes \mathrm{P}_{\beta}}
$$

as illustrated in Fig. 19. 
Fig. 20 We let $h=1, \ell=4$, $\kappa=(0,2,4,6) \in(\mathbb{Z} / 8 \mathbb{Z})^{4}$ and $\beta=\varepsilon_{1}-\varepsilon_{2}$ and $\gamma=\varepsilon_{3}-\varepsilon_{4}$. We picture the element $\operatorname{com}_{\gamma \beta}^{\beta \gamma}$, the corresponding paths are depicted in Fig. 19

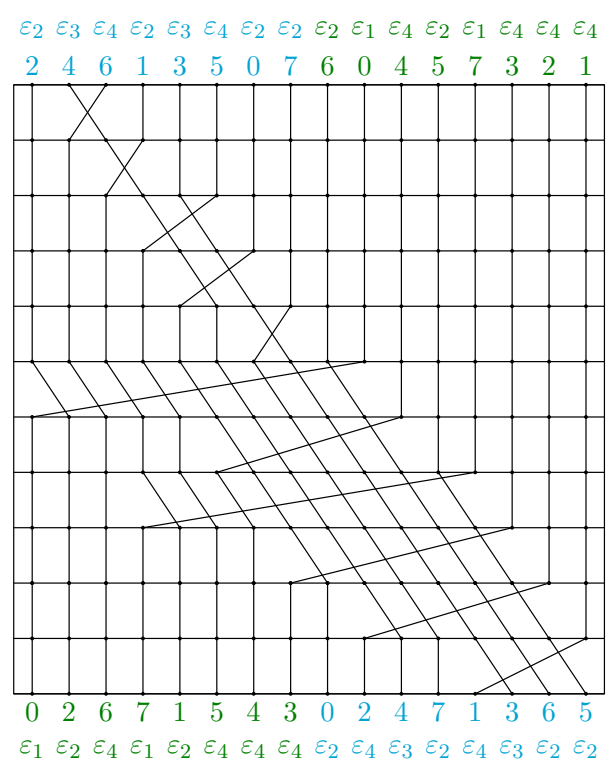

\subsection{Generator morphisms in non-zero degree}

We have already seen how to pass between $S, T \in \operatorname{Std}_{n, \sigma}(\lambda)$ any two reduced paths. We will now see how to inflate a reduced path to obtain a non-reduced path. Given $S, T \in \operatorname{Std}_{n, \sigma}(\lambda)$, we suppose that the former is obtained from the latter by inflating by a path through a single hyperplane $\alpha \in \Pi$. Of course, since $S$ and T have the same shape, this inflation must add an $\mathrm{P}_{\alpha}^{b}$ at some point (and will involve removing an occurrence of $\mathrm{T}_{\emptyset}$ in order to preserve $n$ ). There are two ways which one can approach a hyperplane: from above or from below. Adding an upward/downward occurrence of $\mathrm{P}_{\alpha}^{b}$ corresponds to the spot/fork Soergel generator.

\subsubsection{The spot morphism}

We now define the morphism which corresponds to reflection towards the origin through the hyperplane labelled by $\alpha \in \Pi$. We consider the paths

$\mathrm{P}_{\emptyset}=\left(\varepsilon_{1}, \ldots, \varepsilon_{i-1}, \varepsilon_{i}, \varepsilon_{i+1}, \ldots, \varepsilon_{h}\right)^{b_{\alpha}} \quad \mathrm{P}_{\alpha}^{b}=\left(\varepsilon_{1}, \ldots, \varepsilon_{i-1}, \widehat{\varepsilon}_{i}, \varepsilon_{i+1}, \ldots, \varepsilon_{h}\right)^{b_{\alpha}} \otimes\left(\varepsilon_{i}\right)^{b_{\alpha}}$

examples of these paths are depicted in Fig. 21. We define the KLR-spot to be the element

$$
\operatorname{spot}_{\alpha}^{\emptyset}:=\Upsilon_{\mathrm{P}_{\alpha}^{\mathrm{b}}}^{\mathrm{P}_{\phi}}
$$

which is of degree +1 (corresponding to the unique step of off the $\alpha$-hyperplane). We have already constructed an example of an element $\operatorname{spot}_{\alpha}^{\phi}$ in great detail over the course of Examples 3.6, 3.8 and 3.10 and Fig. 13. 

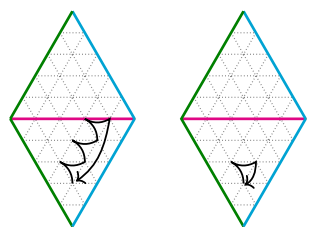

Fig. 21 We let $h=3, \ell=1, e=5$ and $\alpha=\varepsilon_{3}-\varepsilon_{1}$ and we depict the paths $\mathrm{P}_{\alpha}^{b}$ and $\mathrm{P}_{\emptyset}$ (we actually only depict $P_{\emptyset}$ which is a third of the path $\left.P_{\emptyset}\right)$. We have already constructed the corresponding element spot $t_{\alpha}^{\emptyset}$ in great detail over the course of Examples 3.6, 3.8 and 3.10 and Fig. 13

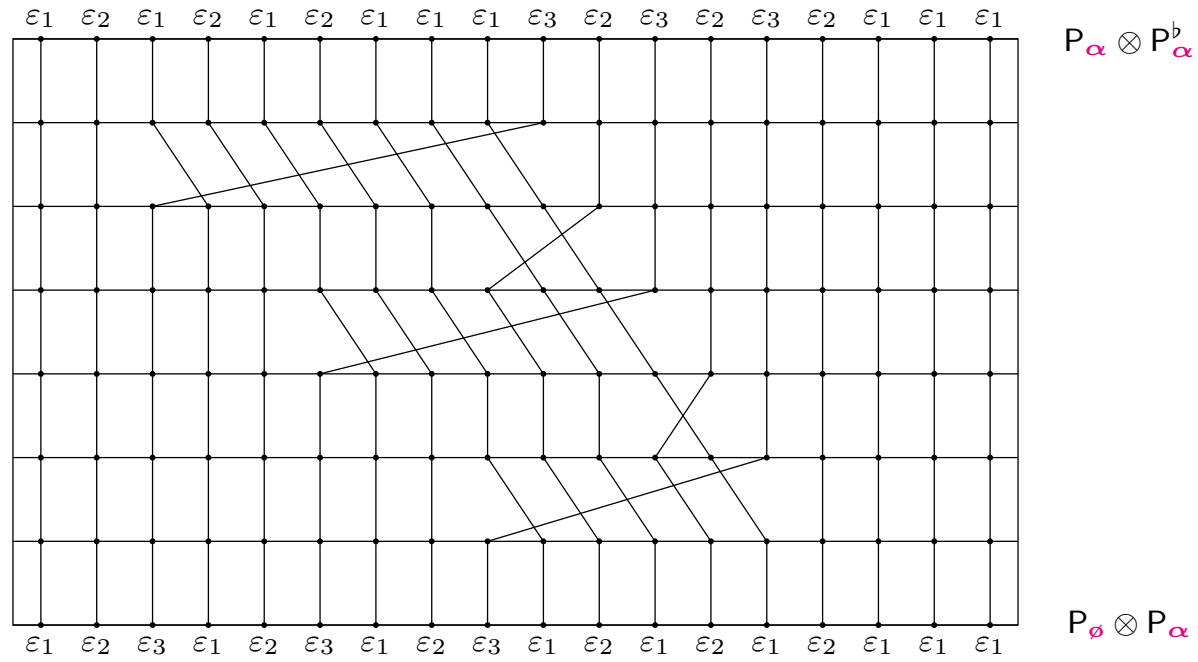

Fig. 22 Fix $\ell=1$ and $h=3$ and $e=5$ and $\alpha=\varepsilon_{3}-\varepsilon_{1}$ (so that $b_{\alpha}=3$ ). We picture the element $\Upsilon_{\mathrm{P}_{\phi} \otimes \mathrm{P}_{\alpha}}^{\mathrm{P}_{\alpha} \otimes \mathrm{P}_{\alpha}^{b}}$. The first 9 and final 4 of the branching coefficients are trivial and so we do not waste trees by picturing all of them. The corresponding paths are pictured in Fig. 23

Fig. 23 Fix $\ell=1$ and $h=3$ and $e=5$ and $\alpha=\varepsilon_{3}-\varepsilon_{1}$ (so that $\left.b_{\alpha}=3\right)$. We depict the paths $\mathrm{P}_{\alpha} \otimes \mathrm{P}_{\alpha}^{\mathrm{b}}$ and $\mathrm{P}_{\emptyset} \otimes \mathrm{P}_{\alpha}$ (although we do not depict the determinant path). The corresponding fork generator is pictured in Fig. 22
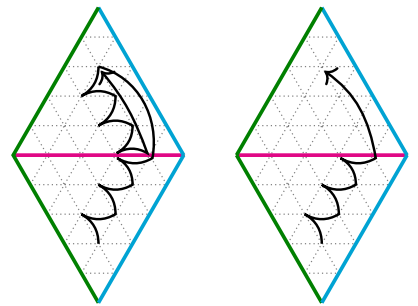

\subsubsection{The fork morphism}

We wish to understand the morphism from $\mathrm{P}_{\alpha} \otimes \mathrm{P}_{\alpha}^{b}$ to $\mathrm{P}_{\emptyset} \otimes \mathrm{P}_{\alpha}$. We define the KLR-fork to be the elements

$$
\text { fork }_{\alpha \alpha}^{ø \alpha}:=\Upsilon_{\mathrm{P}_{\alpha} \otimes \mathrm{P}_{\alpha}^{b}}^{\mathrm{P}_{\phi} \otimes \mathrm{P}_{\alpha}}
$$

as illustrated in Fig. 22. The element fork ${ }_{\alpha \alpha}^{ø \alpha}$ is of degree -1 . 

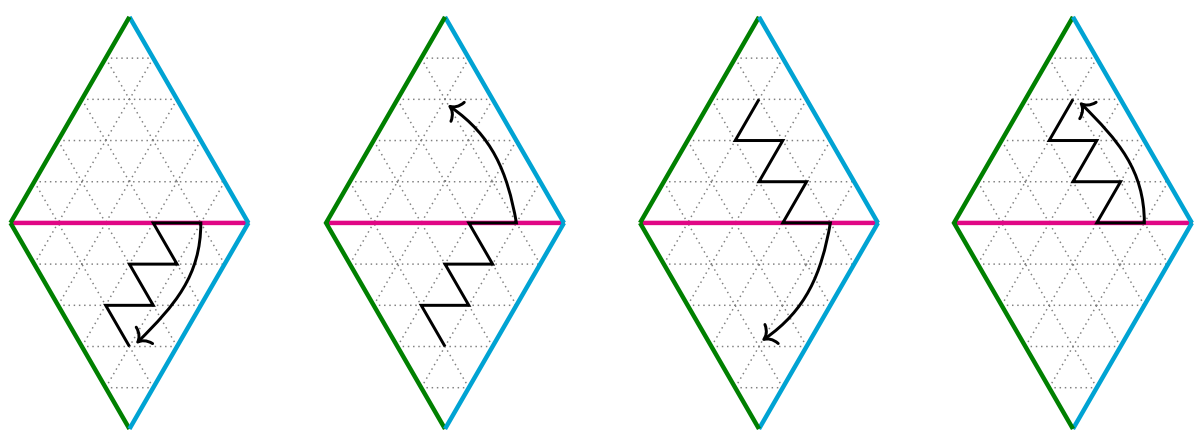

Fig. 24 The first (respectively last) two paths are $S_{\alpha}$ and $P_{\alpha}^{b}$ originating in an alcove with $\alpha$ labelling an upper (respectively lower) wall. Here we take the convention that the origin is below the pink hyperplane. The degrees of these paths are 1, 0, $0,-1$ respectively. We call these paths $U_{0}, U_{1}, D_{0}$, and $D_{1}$ respectively

\subsection{Light leaves for the Bott-Samelson truncation}

We now rewrite the truncated basis of Theorem 3.13 in terms of the Bott-Samelson generators (thus showing that these are, indeed, generators of the truncated algebra). Of course, the idempotent of Eq. (4.1) is specifically chosen so that the truncated algebra

$$
\mathrm{f}_{n, \sigma}\left(\mathcal{H}_{n}^{\sigma} / \mathcal{H}_{n}^{\sigma} \mathrm{y}_{\underline{h}} \mathcal{H}_{n}^{\sigma}\right) \mathrm{f}_{n, \sigma}
$$

has basis indexed by the (sub)set of alcove-tableaux (and this basis is simply obtained from that of Theorem 2.24 by truncation). It only remains to illustrate how the reduced-path-vectors can be chosen to mirror the construction of paths in $\operatorname{Std}_{n, \sigma}(\lambda)$ via concatenation.

We can extend a path $\mathrm{T}^{\prime} \in \operatorname{Std}_{n, \sigma}(\lambda)$ to obtain a new path $\mathrm{T}$ in one of three possible ways

$$
\mathrm{T}=\mathrm{T}^{\prime} \otimes \mathrm{P}_{\alpha} \quad \mathrm{T}=\mathrm{T}^{\prime} \otimes \mathrm{P}_{\alpha}^{\mathrm{b}} \quad \mathrm{T}=\mathrm{T}^{\prime} \otimes \mathrm{P}_{\emptyset}
$$

for some $\alpha \in \Pi$. The first two cases each subdivide into a further two cases based on whether $\alpha$ is an upper or lower wall of the alcove containing $\lambda$. These four cases are pictured in Fig. 24 (for $\mathrm{S}_{\emptyset}$ we refer the reader to Fig. 14). Any two reduced paths $\mathrm{P}_{\underline{w}}, \mathrm{P}_{\underline{v}} \in \operatorname{Std}_{n, \sigma}(\lambda)$ can be obtained from one another by some iterated application of hexagon, adjustment, and commutativity permutations. We let

$$
\operatorname{rex}_{P_{\underline{w}}^{\underline{v}}}^{P_{\underline{v}}}
$$

denote the corresponding path-morphism in the algebras $\mathcal{H}_{n}^{\sigma} / \mathcal{H}_{n}^{\sigma} \mathrm{y}_{h} \mathcal{H}_{n}^{\sigma}$ (so-named as they permute reduced expressions). In the following construction, we will assume that the elements $c_{\mathrm{T}^{\prime}}^{\mathrm{S}^{\prime}}$ exist for any choice of reduced path $\mathrm{S}^{\prime}$. We then extend $\mathrm{S}^{\prime}$ using one of the $U_{0}, U_{1}, D_{0}$, and $D_{1}$ paths (which puts a restriction on the form of the reduced expression) but then use a "rex move" to obtain cellular basis elements "glued together" along an idempotent corresponding to an arbitrary reduced path.

Definition 4.11 Suppose that $\lambda$ belongs to an alcove which has a hyperplane labelled by $\alpha$ as an upper alcove wall. Let $\mathrm{T}^{\prime} \in \operatorname{Std}_{n, \sigma}(\lambda)$. If $\mathrm{T}=\mathrm{T}^{\prime} \otimes \mathrm{P}_{\alpha}$ then we inductively define

$$
c_{\mathrm{P}}^{\mathrm{T}}=\left(c_{\mathrm{P}^{\prime}}^{\mathrm{T}^{\prime}} \otimes e_{\mathrm{P}_{\alpha}}\right) \operatorname{rex}_{\mathrm{P}}^{\mathrm{P}^{\prime} \otimes \mathrm{P}_{\alpha}} \text {. }
$$

If $T=T^{\prime} \otimes P_{\alpha}^{b}$ then we inductively define

$$
c_{\mathrm{P}}^{\top}=\left(c_{\mathrm{P}^{\prime}}^{\mathrm{T}^{\prime}} \otimes \operatorname{spot}_{\emptyset}^{\alpha}\right) \operatorname{rex}_{\mathrm{P}}^{\mathrm{P}^{\prime} \otimes \mathrm{P}_{\emptyset}} .
$$


Now suppose that $\lambda$ belongs to an alcove which has a hyperplane labelled by $\alpha$ as a lower alcove wall. Thus we can choose $\mathrm{P}_{\underline{v}} \otimes \mathrm{P}_{\alpha}=\mathrm{P}^{\prime} \in \operatorname{Std}(\lambda)$. For $\mathrm{T}=\mathrm{T}^{\prime} \otimes \mathrm{P}_{\alpha}$, we inductively define

$$
c_{\mathrm{P}}^{T}=\left(c_{\mathrm{P}^{\prime}}^{T^{\prime}} \otimes e_{\mathrm{P}_{\alpha}}\right)\left(\mathrm{e}_{\mathrm{P}_{\underline{v}}} \otimes\left(\text { fork }_{\alpha \emptyset}^{\alpha \alpha} \circ \operatorname{spot}_{\emptyset}^{\alpha}\right)\right) \operatorname{rex}_{\mathrm{P}}^{\mathrm{P}_{\underline{\underline{v}} \phi \emptyset}}
$$

and if $T=T^{\prime} \otimes P_{\alpha}^{b}$ then then we inductively define

$$
c_{\mathrm{P}}^{T}=\left(c_{\mathrm{P}^{\prime}}^{T^{\prime}} \otimes e_{\mathrm{P}_{\alpha}}\right)\left(e_{\mathrm{P}_{\underline{v}}} \otimes \text { fork }_{\alpha \emptyset}^{\alpha \alpha}\right) \operatorname{rex}_{\mathrm{P}}^{\mathrm{P}_{\underline{\underline{v}} \alpha \emptyset}} .
$$

Theorem 4.12 (The Libedinsky-Williamson light leaves basis) Given weakly increasing $\sigma \in \mathbb{Z}^{\ell}$, we let $\underline{h}=\left(h_{0}, \ldots, h_{\ell-1}\right) \in \mathbb{N}^{\ell}$ be such that $h_{m} \leqslant \sigma_{m+1}-\sigma_{m}$ for $0 \leqslant m<\ell-1$ and $h_{\ell-1}<e+\sigma_{0}-\sigma_{\ell-1}$. Suppose that $n$ is divisible by $h$. For each $\lambda \in \mathscr{P}_{\underline{h}}(n, \sigma)$ we fix an arbitrary reduced path $\mathrm{P}_{\lambda} \in \operatorname{Std}_{n, \sigma}(\lambda)$. The algebra $\mathrm{f}_{n, \sigma}\left(\mathcal{H}_{n}^{\sigma} / \mathcal{H}_{n}^{\sigma} \mathrm{y}_{\underline{h}} \mathcal{H}_{n}^{\sigma}\right) \mathrm{f}_{n, \sigma}$ is quasi-hereditary with graded integral cellular basis

$$
\left\{c_{\mathrm{P}_{\lambda}}^{\mathrm{S}} c_{\mathrm{T}}^{\mathrm{P}_{\lambda}} \mid \mathrm{P}, \mathrm{T} \in \operatorname{Std}_{n, \sigma}(\lambda), \lambda \in \mathscr{P}_{\underline{h}}(n, \sigma)\right\}
$$

with respect to the ordering $\succcurlyeq$ on $\mathscr{P}_{\underline{h}}(n)$, the anti-involution $*$ given by flipping a diagram through the horizontal axis and the map $\operatorname{deg}: \operatorname{Std}_{n, \sigma}(\lambda) \rightarrow \mathbb{Z}$.

Proof Suppose that $\mathrm{Q}, \mathrm{U} \in \operatorname{Std}_{k, \sigma}(v)$ with $\mathrm{Q}$ reduced and $k<n$ divisible by $h$. By induction, we may assume that $c_{\mathrm{U}}^{\mathrm{Q}}=\Upsilon_{\mathrm{U}}^{\mathrm{P}}$ for some reduced path vector $\underline{\mathrm{P}}_{\mathrm{U}}$ such that $\underline{\mathrm{P}}_{\mathrm{U}}=\left(\mathrm{P}_{\mathrm{U}, 0}, \mathrm{P}_{\mathrm{U}, 1}, \ldots, \mathrm{P}_{\mathrm{U}, k}\right)$ with $\mathrm{P}_{\mathrm{U}, k}=\mathrm{Q}$. By Theorem 3.13 and our inductive assumption, the result holds for all $k<n$ divisible by $h$. Now suppose that $\lambda \in \mathscr{P}_{\underline{h}}(n, \sigma)$ and that $\lambda$ belongs to an alcove, $A_{\lambda}$, which has a hyperplane labelled by $\alpha$ and that $\mu=\lambda \cdot s_{\alpha}$. We now reconstruct the element $c_{\mathrm{T}}^{\mathrm{P}}$ in terms of the basis of modified branching coefficients (as in Theorem 3.13) with $P:=P_{\lambda}$ reduced and $T$ equal to either $U \otimes P_{\alpha}$ or $U \otimes P_{\alpha}^{b}$. This amounts to defining a reduced path vector,

$$
\underline{\mathrm{P}}_{\mathrm{T}}=\left(\underline{\mathrm{P}}_{\mathrm{U}}, \mathrm{P}_{\mathrm{T}, k+1}, \mathrm{P}_{\mathrm{T}, k+2}, \ldots, \mathrm{P}_{\mathrm{T}, n}\right)
$$

for which $c_{\mathrm{T}}^{\mathrm{P}}=\Upsilon_{\overline{\mathrm{T}}}^{\mathrm{P}_{\mathrm{T}}}$. To do this, we simply set

$$
\mathrm{P}_{\mathrm{T}, j}= \begin{cases}\left(\mathrm{Q} \otimes \mathrm{P}_{\alpha}\right) \downarrow \leqslant j & \text { if } \mathrm{T}=\mathrm{U} \otimes \mathrm{P}_{\alpha} \text { and } k<j<n \\ \left(\mathrm{Q} \otimes \mathrm{P}_{\alpha}^{b}\right) \downarrow \leqslant j & \text { if } \mathrm{T}=\mathrm{U} \otimes \mathrm{P}_{\alpha}^{b} \text { and } k<j<n \\ \mathrm{P} & \text { if } j=n .\end{cases}
$$

To summarise: we incorporate the "rex" move into the final branching coefficient (and all other branching coefficients are left unmodified). Choosing the reduced path vectors in this fashion, we obtain the required basis as a special case of Theorem 3.13 .

We have shown that we can write a basis for our algebra entirely in terms of the elements

$$
e_{\mathrm{P}_{\alpha}}, \quad \text { fork }_{\alpha \alpha}^{\alpha \emptyset}, \quad \operatorname{spot}_{\alpha}^{\emptyset}, \quad \operatorname{hex}_{\alpha \beta \alpha}^{\beta \alpha \beta}, \quad \operatorname{com}_{\beta \gamma}^{\gamma \beta}, \quad e_{\mathrm{P}_{\emptyset}}, \text { and } \operatorname{adj}_{\alpha \emptyset}^{\emptyset \alpha}
$$

for $\alpha, \beta, \gamma \in \Pi$ such that $\alpha$ and $\beta$ label an arbitrary pair of non-commuting reflections and $\beta$ and $\gamma$ label an arbitrary pair of commuting reflections. Thus we deduce the following:

\section{Corollary 4.13 Theorem $B$ of the introduction holds.}

Acknowledgements The first and third authors thank the Institut Henri Poincaré for hosting us during the thematic trimester on representation theory. The first author was funded by EPSRC grant EP/V00090X/1 and the third author was funded by the Royal Commission for the Exhibition of 1851. The authors would like to express their gratitude to the referee for their incredibly helpful comments and careful reading of the paper. 
Open Access This article is licensed under a Creative Commons Attribution 4.0 International License, which permits use, sharing, adaptation, distribution and reproduction in any medium or format, as long as you give appropriate credit to the original author(s) and the source, provide a link to the Creative Commons licence, and indicate if changes were made. The images or other third party material in this article are included in the article's Creative Commons licence, unless indicated otherwise in a credit line to the material. If material is not included in the article's Creative Commons licence and your intended use is not permitted by statutory regulation or exceeds the permitted use, you will need to obtain permission directly from the copyright holder. To view a copy of this licence, visit http://creativecommons.org/licenses/by/4.0/.

\section{References}

1. Bowman, C., Cox, A.G.: Modular decomposition numbers of cyclotomic Hecke and diagrammatic Cherednik algebras: a path theoretic approach. Forum Math. Sigma 6, e11 (2018)

2. Bowman, C., Cox, A., Hazi, A.: Path isomorphisms between quiver Hecke and diagrammatic BottSamelson endomorphism algebras. Preprint, arXiv:2005.02825

3. Brundan, J., Kleshchev, A.: Blocks of cyclotomic Hecke algebras and Khovanov-Lauda algebras. Invent. Math. 178(3), 451-484 (2009). (MR2551762)

4. Brundan, J., Kleshchev, A., Wang, W.: Graded Specht modules. J. Reine Angew. Math. 655, 61-87 (2011). https://doi.org/10.1515/CRELLE.2011.033

5. Bowman, C.: The many graded cellular bases of Hecke algebras. arXiv:1702.06579

6. Elias, B., Williamson, G.: Soergel calculus. Represent. Theory 20, 295-374 (2016). (MR3555156)

7. $\mathrm{Hu}$, J., Mathas, A.: Graded cellular bases for the cyclotomic Khovanov-Lauda-Rouquier algebras of type A. Adv. Math. 225(2), 598-642 (2010). https://doi.org/10.1016/j.aim.2010.03.002

8. Khovanov, M., Lauda, A.: A diagrammatic approach to categorification of quantum groups I. Represent. Theory 13, 309-347 (2009). (MR2525917)

9. Kleshchev, A.S., Mathas, A., Ram, A.: Universal graded Specht modules for cyclotomic Hecke algebras. Proc. Lond. Math. Soc. (3) 105(6), 1245-1289 (2012). (MR3004104)

10. Libedinsky, N.: Sur la catégorie des bimodules de Soergel. J. Algebra 320(7), 2675-2694 (2008). (MR2441994)

11. Libedinsky, N., Plaza, D.: Blob algebra approach to modular representation theory. Preprint, arXiv: 1801.07200

12. Lobos, D., Plaza, D., Ryom-Hansen, S.: The nil-blob algebra: an incarnation of type $\tilde{A}_{1}$ soergel calculus and of the truncated blob algebra. arXiv:2001.00073

13. Mathas, A.: Iwahori-Hecke algebras and Schur algebras of the symmetric group. University Lecture Series, vol. 15. Am. Math. Soc. Providence (1999). https://doi.org/10.1090/ulect/015

14. Rouquier, R.: 2-Kac-Moody algebras. Preprint, arXiv: 0812.5023

15. Williamson, G.: Schubert calculus and torsion explosion. J. Am. Math. Soc. 30(4), 1023-1046 (2017). (With a joint appendix with Kontorovich, A., McNamara, P.J.: MR3671935)

Publisher's Note Springer Nature remains neutral with regard to jurisdictional claims in published maps and institutional affiliations. 